(C) Copyright 2013

Justin Tittelfitz 



\title{
Thermoacoustic Tomography in Elastic Media
}

\author{
Justin Tittelfitz
}

\author{
A dissertation \\ submitted in partial fulfillment of the \\ requirements for the degree of \\ Doctor of Philosophy \\ University of Washington \\ 2013 \\ Reading Committee: \\ Gunther Uhlmann, Chair \\ Hart Smith, Chair \\ Kenneth Bube \\ Bernard Deconinck
}

Program Authorized to Offer Degree:

Mathematics 



\title{
University of Washington
}

\author{
Abstract \\ Thermoacoustic Tomography in Elastic Media \\ Justin Tittelfitz \\ Co-Chairs of the Supervisory Committee: \\ Gunther Uhlmann \\ Mathematics \\ Hart Smith \\ Mathematics
}

We investigate the problem of recovering the initial displacement $f$ for a solution $u$ of a linear, isotropic, non-homogeneous elastic wave equation, given measurements of $u$ on $[0, T] \times \partial \Omega$, where $\Omega \subset \mathbb{R}^{3}$ is some bounded domain containing the support of $f$. For the acoustic wave equation, this problem is known as thermoacoustic tomography (TAT), and has been well-studied; for the elastic wave equation, the situation is somewhat more subtle, and we give sufficient conditions on the Lamé parameters to ensure that recovery is possible. Following this, we investigate the numerical simulation of this problem. 



\section{TABLE OF CONTENTS}

Page

List of Figures . . . . . . . . . . . . . . . . . . . . iii

Chapter $1: \quad$ Introduction . . . . . . . . . . . . . . . 1

1.1 Thermoacoustic Tomography . . . . . . . . . . . . . . . . 2

Chapter 2: $\quad$ Mathematical Preliminaries . . . . . . . . . . . . . 7

2.1 Microlocal Analysis . . . . . . . . . . . . . . . . . . . . 7

2.1.1 Symbols, phase functions, and Fourier integral operators . . . . . . . 7

2.1.2 The singular support and wave front set of a distribution . . . . . . . 11

2.1.3 The Hamiltonian and propagation of singularities . . . . . . . . . . . 13

2.1.4 The non-trapping hypothesis . . . . . . . . . . . . . . . 18

2.2 Functional Analysis . . . . . . . . . . . . . . . . . . . . . . . . . . . . . 19

2.2.1 The spectrum of a bounded linear operator . . . . . . . . . . 19

2.2 .2 Compact operators . . . . . . . . . . . . . . 22

Chapter 3: Thermoacoustic Tomography in Elastic Media . . . . . . . . . . . . 26

3.1 The Elastic Wave Equation . . . . . . . . . . . . . . . 26

3.2 Unique Continuation and Conditions on the Lamé Parameters . . . . . . . . . 29

3.3 Energy of Initial Data and Solutions . . . . . . . . . . . . . . . . . 34

3.4 Reconstruction . . . . . . . . . . . . . . . . . 36

Chapter 4: Background on Numerical Work . . . . . . . . . . . . . . 41

4.1 Finite Difference Methods . . . . . . . . . . . . . . . . . . . . . . . 41

4.1 .1 Finite differences . . . . . . . . . . . . . . . . 41

4.1 .2 Finite differences on a grid . . . . . . . . . . . . . . 43

4.1.3 Convergence, consistency and stability . . . . . . . . . . . . . 44

4.1 .4 Von Neumann analysis . . . . . . . . . . . . . . . . . . . . . . . . . . . . 46

4.1.5 Two-step methods and stability . . . . . . . . . . . . . . . 49

4.2 Our Methods . . . . . . . . . . . . . . . . . . . . . 51 
4.2 .1 Forward problem . . . . . . . . . . . . . . . . 52

4.2 .2 Elliptic problem . . . . . . . . . . . . . . . . 54

4.2 .3 Time-reversed problem . . . . . . . . . . . . . 55

Chapter $5: \quad$ Numerical Work . . . . . . . . . . . . . . . 57

5.1 Methodology . . . . . . . . . . . . . . . 57

5.1 .1 Boundary Distance Map . . . . . . . . . . . . . . . . . . . 59

5.1 .2 Algorithmic Implementation . . . . . . . . . . . . . . . . . 60

5.2 Results of Numerical Experimentation . . . . . . . . . . . . . 61

5.2 .1 Homogeneous parameters $\left(\lambda_{1}, \mu_{1}\right) \ldots \ldots \ldots \ldots$. . . . . . 61

5.2 .2 Heterogeneous parameters $\left(\lambda_{2}, \mu_{2}\right) \ldots \ldots \ldots \ldots$

5.2.3 Heterogeneous parameters $\left(\lambda_{3}, \mu_{3}\right)$, Lamé hypothesis violated . . . . . 65

5.2.4 Heterogeneous parameters $\left(\lambda_{4}, \mu_{4}\right)$, Lamé hypothesis violated with

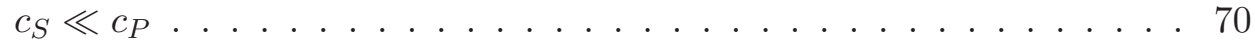

5.3 Anisotropic Elasticity . . . . . . . . . . . . . . . . 70

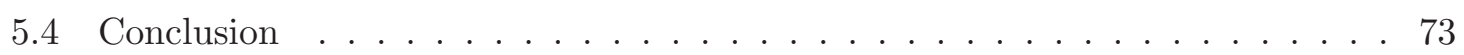

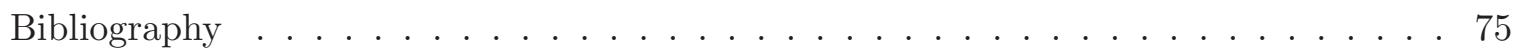




\section{LIST OF FIGURES}

Figure Number $\quad$ Page

1.1 An EM pulse leading to elastic wave propagation . . . . . . . . . . . . 2

2.1 An example of a trapped geodesic. . . . . . . . . . . . . . . . 19

3.1 The solid gray region is $B(0, R) \times(-T, T)$; the dashed region where $u=0$ by finite speed of propagation and time-reversal. . . . . . . . . . 33

5.1 P-wave boundary distance maps for (a) $c_{1, P}: T_{0}=1.330$; (b) $c_{2, P}: T_{0}=$ $1.261 ;$ (c) $c_{3, P}: T_{0}=0.986 ;$ (d) $c_{4, P}: T_{0}=1.888 \ldots \ldots \ldots \ldots$

5.2 S-wave boundary distance maps for (a) $c_{1, S}: T_{1}=1.996$; (b) $c_{2, S}: T_{1}=1.888$;

(c) $c_{3, S}: T_{1}=3.572 \ldots \ldots \ldots \ldots \ldots \ldots \ldots$

5.3 Initial Data . . . . . . . . . . . . . . . . . . . . 61

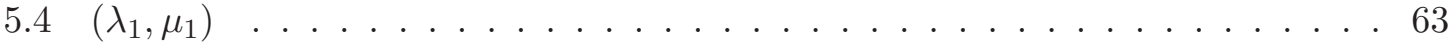

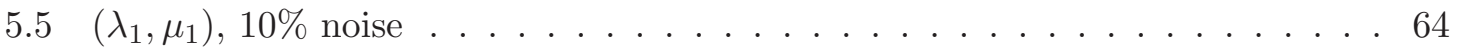

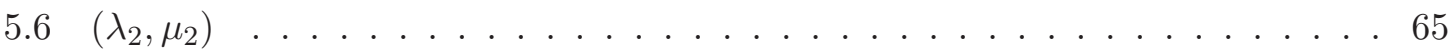

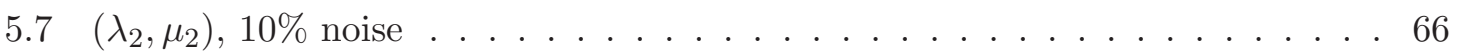

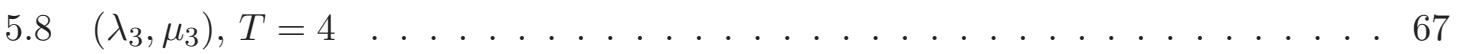

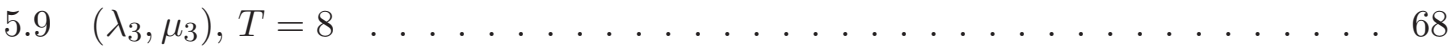

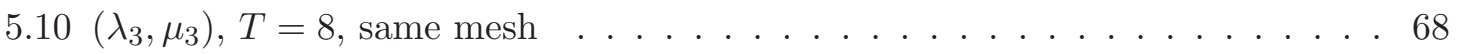

$5.11\left(\lambda_{3}, \mu_{3}\right), T=8$, added noise, same mesh . . . . . . . . . . . . 69

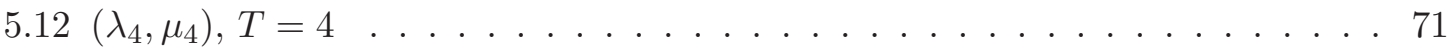

5.13 Anisotropic system . . . . . . . . . . . . . . . . . . . . . . . . . . . . . . . . .

5.14 Anisotropic system with $10 \%$ noise $\ldots \ldots \ldots \ldots$. . . . . . . . 72 


\section{ACKNOWLEDGMENTS}

The author wishes to express sincere appreciation to the University of Washington, his advisors, the computing support staff in the department of mathematics, the NSF for their

generous support (RTG DMS-0838212), and to Katie Oliveras and Francois Monard for their many helpful suggestions. 


\section{DEDICATION}

to my dear wife, Talia 



\section{Chapter 1}

\section{INTRODUCTION}

Throughout this work, we consider the linear, isotropic elastic wave equation and Cauchy initial value problem given by

$$
\begin{cases}\left(\partial_{t}^{2}+P\right) u & =0 \quad \text { in }(0, T) \times \mathbb{R}^{3} \\ \left.u\right|_{t=0} & =f \\ \left.\partial_{t} u\right|_{t=0} & =0\end{cases}
$$

where $u=\left(u_{1}, u_{2}, u_{3}\right)$ is the displacement vector,

$$
-P u=\nabla \cdot\left(\mu(x)\left((\nabla u)+(\nabla u)^{T}\right)\right)+\nabla(\lambda(x) \nabla \cdot u)
$$

$\lambda$ and $\mu$ are the Lamé parameters,

$$
(\nabla u)_{i, j}=\frac{\partial u_{i}}{\partial x_{j}}
$$

is the Jacobian of $u$ and $(\nabla u)^{T}$ is its transpose. Equivalently, we can write

$$
-P u=\mu(\Delta u+\nabla(\nabla \cdot u))+\lambda \nabla(\nabla \cdot u)+(\nabla \cdot u) \nabla \lambda+\sum_{j=1}^{3} \nabla \mu \cdot\left(\nabla u_{j}+\partial_{j} u\right) e_{j} .
$$

We will assume that $\lambda(x)$ and $\mu(x)$ are independent of $t$; we will also assume that they are each positive, in that there is some positive constant $\alpha_{0}$ so that $\lambda, \mu>\alpha_{0}$, and later, we will make further assumptions about bounds and smoothness.

Throughout, we will assume that our initial-data, $f$, is compactly supported in a set $\Omega$, which could, in general, be any bounded domain in $\mathbb{R}^{3}$, though for simplicity, we will take $\Omega=B(0, R)$ for some $R>0$. The objective of thermoacoustic tomography in elastic media is to recover $f$, given the data

$$
\Lambda f:=\left.u\right|_{[0, T] \times \partial \Omega} .
$$




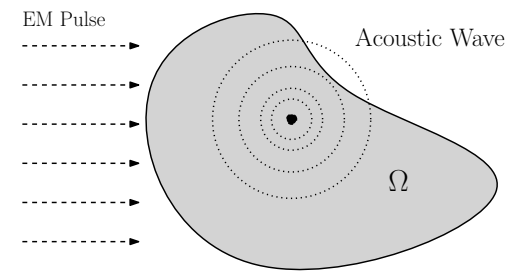

Figure 1.1: An EM pulse leading to elastic wave propagation

In particular, we will show that, if the Lamé parameters satisfy a condition on their relative size (briefly, that $\sup \sqrt{\mu}<3 \inf \sqrt{\lambda+2 \mu}$ ) and one regarding their gradients (to be discussed later), then $f$ is recoverable via a Neumann series. Our method is strongly inspired by the techniques of thermoacoustic tomography for scalar wave equations, and we adapt the timereversal approach used by Stefanov and Uhlmann in [31]. We devote the remainder of this chapter to discussing the existing results and methods in thermoacoustic tomography, and how they motivate the work in this paper.

In the next chapter, we will discuss some mathematical preliminaries needed to prove our main result, which we do in chapter 3. After this, we consider the problem of computationally reconstructing the inital data $f$ using the Neumann series method on the set $\Omega=[-2,2]^{2}$, and show the results of this numerical experimentation.

\subsection{Thermoacoustic Tomography}

Thermoacoustic tomography (hereafter TAT) is a method of medical imaging where the object of interest is exposed to a short electromagnetic (EM) pulse, absorbing some of the EM energy in the process. Because cancerous cells will absorb more of this energy than healthy cells will, it would be diagnostically useful to know the absorption function $a(x)$. To accomplish this, TAT makes use of the elastic expansion in the nearby tissue caused by the energy absorption, as well as the fact that this initial pressure distribution is roughly proportional to the absorption distribution. This initial pressure, in turn, leads to a pressure wave $u(t, x)$ that propagates through the object, and is then measured by transducers located on an observation surface $\Gamma$ surrounding the object for some length of time, the goal being to use this data to reconstruct the initial pressure (see Figure 1.1). In 
much of the literature, the intended application is the imaging of cancer in a human breast, though some authors have written specifically about imaging the brain. This is somewhat more complicated, as the skull introduces a jump discontinuity in the sound speed, but under certain assumptions, recovery is still quite possible (see [32], [26]).

Mathematically, we consider the scalar wave equation and Cauchy initial value problem

$$
\begin{cases}\left(\partial_{t}^{2}+A\right) u & =0 \quad \text { in }(0, T) \times \mathbb{R}^{n} \\ \left.u\right|_{t=0} & =f \\ \left.\partial_{t} u\right|_{t=0} & =0\end{cases}
$$

where $A(x, D)=-c^{2}(x) \Delta$, (or more generally, $A(x, D)=-c^{2}(x) \Delta_{g}$, for some other metric $g$ on $\mathbb{R}^{n}$ ). The initial pressure function $f$ is typically assumed to be compactly supported inside of some bounded domain $\Omega \subset \mathbb{R}^{n}$ (corresponding to the object to be imaged), though in some work, $f$ is taken to be supported in some compact set, or even merely $f \in L^{p}$ for $p>2 n /(n-1)$ (see [1]). The observation surface $\Gamma$ is often taken to be $\partial \Omega$ (sometimes referred to as complete data), though there are also satisfactory results for the case where $\Gamma$ is some other set, such as a portion of $\partial \Omega$ (likewise, incomplete data; see, for instance, [31], [44], [45]). The data one then collects is

$$
\Lambda f:=\{u(t, y): 0 \leq t \leq T, y \in \Gamma\}
$$

and from this, the goal of TAT is to recover $f$.

There are three main recovery methods used in TAT, and their applicability depends largely on the assumptions made about the geometry and physical attributes of the medium (in terms of $\Omega$ and $c(x)$ ) and the observation surface $\Gamma$. We will briefly discuss some of these here; for a detailed account and comparison of these techniques, including their relative advantages and disadvantages, see the excellent survey papers by Hristova, Kuchment and Nguyen [16], or Kuchment and Kunyansky [17], [18].

In the method of filtered backprojection, one assumes that $c$ is constant (i.e. the medium is acoustically homogeneous) and that the observation surface $\Gamma$ is a sphere of radius $R$. We can then recover $f$ through integral formulas such as

$$
f(x)=-\frac{1}{8 \pi^{2} R} \Delta \int_{\Gamma} \frac{h(|y-x|, y)}{|y-x|} d A(y),
$$


where

$$
h(r, y)=\int_{\mathbb{S}^{n-1}} f(y+r \omega) r^{n-1} d \omega, \quad y \in \Gamma
$$

are the spherical integrals of $f$, with $d A$ and $d \omega$ are the surface measures on the respective spheres. For more on the integral geometry approach to TAT, see the work of Agranovsky, Berenstein and Kuchment [1], Finch, Patch and Rakesh [9], Xu and Wang [43], Finch, Haltmeier and Rakesh [8] and Kunyansky [20].

The method of eigenfunction expansion applies to a slightly more general setting, in theory allowing $c$ to be variable, and for $\Gamma$ to be any closed surface (i.e. $\Gamma=\partial \Omega$ for some $\Omega)$. We then seek to write $f$ as a Fourier series

$$
f(x)=\sum f_{k} \psi_{k}(x)
$$

where $\psi_{k}$ are eigenfunctions of the operator $-c^{2}(x) \Delta$ in $\Omega$ with Dirichlet boundary conditions on $\partial \Omega$, and then find the coefficients $f_{k}$ using integral formulas. In [19], Kunyansky showed, in the case $\Gamma$ is a cube and $c$ is constant, that $f$ can be recovered fast and precisely. Of course, for a complicated set $\Omega$, or a variable speed $c(x)$, the eigenfunctions and eigenvalues may not be known, and it is not clear whether this method can be effectively implemented.

The third method (and the method we will eventually use) is known as time-reversal, and was first proposed by Finch, Patch and Rakesh in [9], and first implemented by Burgholzer, Matt, Haltmeier and Paltauf in [4]. Here, $c$ is allowed to be variable (i.e. the medium can be non-homogeneous), and the restrictions on the geometry of $\Gamma$ are far less than those of the other two methods. The key assumption is that there is good local energy decay, meaning that for $f$ compactly supported in $\Omega$, the energy of the solution in $\Omega$ decays sufficiently fast as $t$ increases. To better illustrate the nature of this requirement, we first discuss an ideal case: the constant speed wave equation $\left(\partial_{t}^{2}-\Delta\right) u=0$ in $\mathbb{R}^{+} \times \mathbb{R}^{3}$. In this setting, Huygen's principle would apply, and we would know that for some $\widetilde{T}, u(t, x)=0$ inside $\Omega$ for $t>\widetilde{T}$. 
In this case, we could recover $f$ by considering solutions of the initial value problem

$$
\left\{\begin{array}{l}
\left(\partial_{t}^{2}-\Delta\right) v=0 \quad \text { in } \mathbb{R}^{+} \times \mathbb{R}^{n}, \\
\left.v\right|_{t=\widetilde{T}}=\left.v_{t}\right|_{t=\widetilde{T}}=0 \\
\left.v\right|_{\partial \Omega}=g(t, y)
\end{array}\right.
$$

where $g=\Lambda f$, and then solving the problem in the reverse time direction, since $v(0, x)=$ $f(x)$ by uniqueness.

Of course, Huygen's principle does not apply in the general setting, but there is a useful analog which still leads to strong results. To ensure the kind of energy decay we need, we will assume that the speed is non-trapping, meaning that all rays starting in $\Omega$ leave in finite time, and that the supremum of these times $T(\Omega)$ is also finite. We will discuss this further in the next chapter.

Because we can no longer assume that $u$ eventually vanishes inside $\Omega$, it would be incorrect to think that, for any finite time $\widetilde{T}$, solutions $v$ of $(1.2)$ will satisfy $v(0, x)=f(x)$, at least not exactly. To alleviate this, some authors have replaced the boundary condition by $\left.v\right|_{\partial \Omega}=\chi(t) g(t, y)$, where $\chi$ is a smooth cutoff function vanishing near $\widetilde{T}>T(\Omega)$, and $\chi=1$ near $(-\infty, T(\Omega))$ (see [15]). In [31], Stefanov and Uhlmann used a different timereversal method (this will be discussed thoroughly in a later chapter), and showed that, for the acoustic wave equation with variable sound speed, it is possible to invert $\Lambda$ using a Neumann series. Subsequently, in [26], Qian, Stefanov, Uhlmann and Zhou went on to give a specific numerical algorithm for recovery based on this theoretical understanding. In this paper, we will follow the method of [31] when possible, assuming that the absorption of EM energy and subsequent elastic tissue expansion lead to elastic wave propagation instead of acoustic.

The differences between scalar equations and systems will introduce some difficulties, the main difficulty being the unique continuation problem; i.e., determining when specifying Cauchy data on a hypersurface $S \subset \mathbb{R} \times \mathbb{R}^{3}$ is sufficient to uniquely determine the solution in a neighborhood of $S$. For the classical case (where the coefficients are smooth), this is Holmgren's theorem (see, for instance, [38]). In the case of non-smooth coefficients for a scalar wave equation, there are good results due to Tataru (see [35],[36]). For the static 
Lamé system (i.e. the elliptic system $P u=0$ ), Lin, Nakamura, Uhlmann and Wang have recently shown (in [23]) that there is a strong unique continuation principle; specifically, if $\lambda \in L^{\infty}$ and $\mu \in C^{0,1}$, in $\mathbb{R}^{n}$ with $n \geq 2$, if a solution is zero in a neighborhood of any point, it is identically zero. For the elastic wave equation, however, the situation is somewhat more subtle; while some results exist, we will have to make assumptions about the Lamé parameters to acquire satisfactory results. This will be discussed further in Chapter 3, following which we will prove our main result. 


\section{Chapter 2}

\section{MATHEMATICAL PRELIMINARIES}

In this chapter, we discuss some of the preliminaries needed to understand and prove the main results of this work. We briefly cover the topics of microlocal analysis and some functional analysis, including the spectral theory of compact operators.

\subsection{Microlocal Analysis}

Suppose $f$ is a function which fails to be smooth on a set $\Omega$, and $u$ is the solution of a wave equation using $f$ as its initial data. What can we say generally about the smoothness of $u$, and how do the specific locations of $f$ 's singularities influence those of $u$ ? In this section, we seek to make precise the notion of a function's singularities, and give satisfactory answers to the questions just posed.

This topic is usually known as microlocal analysis, and has been discussed extensively in the literature; see for instance [6], [11], [12]-[14], and [41] among many others. We will assume some familiarity with manifolds and differential geometry in this section.

Throughout this section, let $X$ and $Y$ be open sets in $\mathbb{R}^{n}$ and $\mathbb{R}^{m}$ (more generally, we can let $X$ and $Y$ be $n$ - and $m$-dimensional smooth manifolds, but for the sake of simplicity, we restrict our attention to subsets of Euclidean space).

\subsubsection{Symbols, phase functions, and Fourier integral operators}

A function $a(x, \theta) \in C^{\infty}\left(X \times \mathbb{R}^{N}\right)$ is called a symbol of order $m$ if, for every multi-index $\alpha=\left(\alpha_{1}, \ldots, \alpha_{N}\right)$, there exists a constant $C$ depending on $\alpha$ such that

$$
\left|\partial_{\theta}^{\alpha} a(x, \theta)\right| \leq C(1+|\theta|)^{m-|\alpha|}
$$

for all $(x, \theta) \in X \times \mathbb{R}^{N}$. We call the collection of all such symbols $S^{m}\left(X \times \mathbb{R}^{N}\right)$. 
A real-valued function $\phi(x, \theta) \in C^{\infty}\left(X \times \mathbb{R}^{N} \backslash\{0\}\right)$ such that $\nabla \phi$ is non-vanishing, and such that $\phi(x, \lambda \theta)=\lambda \phi(x, \theta)$ for all $\lambda>0$ (i.e. $\phi$ is positively homogeneous of degree 1 ) is called a non-degenerate phase function.

If $a \in S^{m}\left(X \times Y \times \mathbb{R}^{N}\right)$ is a symbol, and $\phi \in C^{\infty}\left(X \times Y \times \mathbb{R}^{N} \backslash\{0\}\right)$ is a non-dengenerate phase function, we can define an operator $A$, called a Fourier integral operator of order $m$ (or FIO), by

$$
A u(x)=\iint e^{i \phi(x, y, \theta)} a(x, y, \theta) u(y) d y d \theta
$$

We note that it is not clear in advance that this integral converges - an obstruction we will have to overcome. First, we consider an example.

Let $X=Y=\mathbb{R}^{n}, N=n$, and, to be suggestive, replace $\theta$ by $\xi$. Let $\phi(x, y, \xi)=\langle x-y, \xi\rangle$, and let $a(x, y, \xi)=|\xi|^{2}$. It is easy to verify $\nabla \phi \neq 0$, that it is positively homogeneous, and that $a$ is a symbol of order 2 .

Then, for this choice of phase function and symbol,

$$
\begin{aligned}
A u(x) & =\iint e^{i\langle x-y, \xi\rangle}|\xi|^{2} u(y) d y d \xi \\
& =\int e^{i\langle x, \xi\rangle}|\xi|^{2} \int e^{-i\langle y, \xi\rangle} u(y) d y d \xi \\
& =\int e^{i\langle x, \xi\rangle}|\xi|^{2} \widehat{u}(\xi) d \xi \\
& =\mathcal{F}^{-1}\left(|\xi|^{2} \widehat{u}\right) \\
& =\Delta u,
\end{aligned}
$$

i.e. $A$ is just the Laplacian (here, $\mathcal{F}^{-1}$ is just the inverse Fourier transform, and we omit factors of $2 \pi)$.

In fact, every differential operator $A(x, D)=\sum_{|\alpha| \leq m} a_{\alpha}(x) D_{x}^{\alpha}$ of order $m$ (where $D_{x_{j}}=$ $\left.-i \frac{\partial}{\partial x_{j}}\right)$ is a Fourier integral operator of order $m$, with symbol $a(x, \xi)=\sum_{|\alpha| \leq m} a_{\alpha}(x) \xi^{\alpha}$ and phase function $\phi(x, y, \xi)=\langle x-y, \xi\rangle$. More generally, if $\phi(x, y, \xi)=\langle x-y, \xi\rangle$, and $a \in S^{m}\left(X \times X \times \mathbb{R}^{n}\right)$, we say $A$ is a pseudodifferential operator of order $m$.

Now we show this is well defined for more general $a$ and $\phi$. Let $\mathcal{D}^{\prime}(X)$ be the space of distributions on $X$, i.e. the dual space of $C_{0}^{\infty}(X)$. We will use $\langle\cdot, \cdot\rangle$ to indicate this duality. 
Given a symbol $a(x, \theta)$ and phase function $\phi(x, \theta)$, we formally define a distribution $I(a, \phi)$ by

$$
\langle I(a, \phi), u\rangle=\iint e^{i \phi(x, \theta)} a(x, \theta) u(x) d x d \theta
$$

that is,

$$
I(a, \phi)=\int e^{i \phi(x, \theta)} a(x, \theta) d \theta
$$

Under some circumstances, it is easy to verify this is a distribution. In particular, if $a$ decays rapidly enough as $|\theta| \rightarrow \infty$, this is trivial:

Proposition 2.1.1. If $a \in S^{m}\left(X \times \mathbb{R}^{N}\right)$ for $m<-N$, and $\phi$ is a non-degenerate phase function, then the integral defining $\langle I(a, \phi), u\rangle$ is absolutely convergent for every $u \in C_{0}^{\infty}(X)$.

More generally, we have the following theorem (we give the version stated in [11]). Here, we write $S^{m}$ instead of $S^{m}\left(X \times \mathbb{R}^{N}\right)$ for convenience, and we take $S^{\infty}=\cup_{m \in \mathbb{R}} S^{m}$, $S^{-\infty}=\cap_{m \in \mathbb{R}} S^{m}$.

Theorem 2.1.2. If $\phi$ is a non-degenerate phase function, then there is a unique way of defining $I(a, \phi) \in \mathcal{D}^{\prime}(X)$ for $a \in S^{\infty}$ so that $I(a, \phi)$ is given by (2.2) for a $\in S^{m}$ with $m<-N$, and for every $m \in \mathbb{R}$, the map $S^{m} \rightarrow \mathcal{D}^{\prime}(X): a \mapsto I(a, \phi)$ is continuous.

A full proof is given in [11], but briefly, this hinges on the following lemma (also stated as in $[11])$ :

Lemma 2.1.3. There exist $a_{j} \in S^{0}, b_{j}, c \in S^{-1}$ such that the differential operator

$$
L=\sum a_{j}(x, \theta) \frac{\partial}{\partial \theta_{j}}+\sum b_{j}(x, \theta) \frac{\partial}{\partial x_{j}}+c(x, \theta)
$$

satisfies $L^{t}\left(e^{i \phi}\right)=e^{i \phi}$, where $L^{t}$ is the real transpose of $L$.

Armed with such an operator, we have for any $a \in S^{-\infty}, u \in C_{0}^{\infty}(X), k \in \mathbb{N}$

$$
\begin{aligned}
\langle I(a, \phi), u\rangle & =\iint e^{i \phi(x, \theta)} a(x, \theta) u(x) d x d \theta \\
& =\iint\left(\left(L^{t}\right)^{k} e^{i \phi(x, \theta)}\right) a(x, \theta) u(x) d x d \theta \\
& =\iint e^{i \phi(x, \theta)} L^{k}(a(x, \theta) u(x)) d x d \theta
\end{aligned}
$$


by means of repeated integration by parts. We observe that, if $a \in S^{m}$, then $L^{k}(a u) \in S^{m-k}$, and if we choose $k$ so that $m-k<-N$, and define

$$
\left\langle I_{k}(a, \phi), u\right\rangle:=\iint e^{i \phi(x, \theta)} L^{k}(a(x, \theta) u(x)) d x d \theta
$$

we can see $I_{k}(a, \phi) \in \mathcal{D}^{\prime}(X)$ (as in the proposition). A density argument then allows us to define $I(a, \phi)=I_{k}(a, \phi)$ for any $k$ such that $m-k<-N$.

Returning to phase functions and symbols on $X \times Y \times \mathbb{R}^{N}$, we now have $k(x, y)=$ $\int e^{i \phi(x, y, \theta)} a(x, y, \theta) d \theta \in \mathcal{D}^{\prime}(X \times Y)$. We now appeal to the Schwartz kernel theorem, a fundamental result in distribution theory (see e.g. [37] or [42]).

Theorem 2.1.4 (Schwartz Kernel Theorem). To every bounded linear map $A: C_{0}^{\infty}(Y) \rightarrow$ $\mathcal{D}^{\prime}(X)$ there corresponds a unique distribution $k \in \mathcal{D}^{\prime}(X \times Y)$ such that

$$
\langle A u, v\rangle=\langle k, u \otimes v\rangle=\iint k(x, y) u(y) v(x) d x d y
$$

for all $u \in C_{0}^{\infty}(Y), v \in C_{0}^{\infty}(X)$. Further, A extends continuously to a map $\mathcal{E}^{\prime}(Y) \rightarrow C^{\infty}(X)$ if and only if $k \in C^{\infty}(X \times Y)$. Such an operator is called a smoothing operator (or regularlizing operator).

So, by this theorem, the distribution $k(x, y) \in \mathcal{D}^{\prime}(X \times Y)$ corresponds to a unique, continuous map $A: C_{0}^{\infty}(Y) \rightarrow \mathcal{D}^{\prime}(X)$, given by

$$
A u(x)=\int k(x, y) u(y) d y
$$

and so Fourier integral operators make sense as maps $C_{0}^{\infty}(Y) \rightarrow \mathcal{D}^{\prime}(X)$.

Furthermore, we can extend the domain/range under some conditions; again this is stated and proved in [11].

Theorem 2.1.5. Let $\phi \in C^{\infty}\left(X \times Y \times \mathbb{R}^{N} \backslash\{0\}\right), a \in S^{m}\left(X \times Y \times \mathbb{R}^{N}\right)$, and $A$ be defined by (2.1). Then,

1. If, for every $x \in X,(y, \theta) \mapsto \phi(x, y, \theta)$ is a non-degenerate phase function, then $A$ is continuous from $C_{0}^{\infty}(Y) \rightarrow C^{\infty}(X)$. 
2. If, for every $y \in Y,(x, \theta) \mapsto \phi(x, y, \theta)$ is a non-degenerate phase function, then $A$ has a unique continuous extension $\mathcal{E}^{\prime}(Y) \rightarrow \mathcal{D}^{\prime}(X)$.

From this theorem, we note that since $\nabla_{x, \xi}\langle x-y, \xi\rangle$ and $\nabla_{x, \xi}\langle x-y, \xi\rangle$ are non-zero when $\xi \neq 0$, pseudodifferential operators are always continuous as maps $C_{0}^{\infty} \rightarrow C^{\infty}$ and have continuous extensions $\mathcal{E}^{\prime} \rightarrow \mathcal{D}^{\prime}$.

We conclude this section by defining the principal symbol of an FIO. First, if $A$ is a differential operator $A(x, D)=\sum_{|\alpha| \leq m} a_{\alpha}(x) D_{x}^{\alpha}$ (so, with symbol $\left.a(x, \xi)=\sum_{|\alpha| \leq m} a_{\alpha}(x) \xi^{\alpha}\right)$, its principal symbol is just $a_{m}(x, \xi)=\sum_{|\alpha|=m} a_{\alpha}(x) \xi^{\alpha}$, i.e. the highest order term(s).

More generally, any symbol $a \in S^{m}$ has a unique asymptotic expansion $a \sim \sum_{j=-\infty}^{m} a_{j}$ with each $a_{j} \in S^{j}$ (see [11], [6], etc. for a proof). In this case, the symbol of the corresponding FIO is just $a_{m}$. It is easy to see this generalizes the definition given for differential operators.

\subsubsection{The singular support and wave front set of a distribution}

Instinctively, the singularities of a function are just the points where it fails to be smooth. Precisely, we say that $x_{0}$ is not in the singular support of $f($ denoted sing supp $(f))$ if there exists a smooth, compactly supported function $\phi$ with $\phi\left(x_{0}\right) \neq 0$ so that $\phi f$ is smooth. Intuitively, if $f$ is smooth at $x_{0}$, there is a neighborhood of $x_{0}$ where $f$ is smooth, and so if $\phi$ is a smooth bump function for that neighborhood, $\phi f$ will be smooth regardless of $f$ 's behavior away from this neighborhood.

According to the Paley-Wiener theorem, if a function $f$ is smooth, then its Fourier transform decays faster than any polynomial. Precisely, if $f$ is smooth, then

$$
|\widehat{f}(\xi)| \leq C_{N}(1+|\xi|)^{-N}
$$

for all positive $N$ and every $\xi$, for some constant $C_{N}$ (depending only on $N$ ). Thus, we can reformulate the singular support of $f$ in terms of the decay of $\phi f$ in the following proposition (which is trivial to prove): 
Proposition 2.1.6. A point $x_{0} \notin \operatorname{sing} \operatorname{supp}(f)$ if and only if there exists a function $\phi \in$ $C_{0}^{\infty}(X)$ with $\phi\left(x_{0}\right) \neq 0$ so that

$$
|\widehat{\phi f}(\xi)| \leq C_{N}(1+|\xi|)^{-N}
$$

for all positive $N$ and every $\xi$, for some constant $C_{N}$.

If we wish to be more precise, we might seek to describe not only where these singularites occur, but also in what direction. To informally illustrate this, imagine standing atop the characteristic function of the closed unit square in $\mathbb{R}^{2}$, at the point $(1,1 / 2)$. Clearly, the function is singular here: if we move at all east (i.e., any amount in the positive $x_{1}$ direction), we will "fall off". However, if we restrict our motion to only north/south (and avert our eyes from the cliff just to our side) we might never notice the discontinuity. Thus, in some sense, this function is singular in one direction, but not the other.

One way we might attempt to describe the direction of singularities is by means of the frequency set of $f$; we say that $\xi_{0}$ is not in the frequency set of $f$, denoted $\Sigma(f)$, if there exists a conic neighborhood $\Gamma$ of $\xi$ such that, for all $N$ positive, there exists $C_{N}$ so that

$$
|\widehat{u}(\xi)| \leq C_{N}(1+|\xi|)^{-N}
$$

for all $\xi \in \Gamma$.

Of course, this only tells us the direction of singularities, and not their locations. If we wish to properly localize in both position and frequency space, we can do so precisely by introducing the wave front set of a distribution. The key idea is to first localize in position space by using bump functions, and then look at the resulting singular frequencies. In particular, if we multiply a distribution by a smooth bump function, we can only refine the singular directions, as demonstrated by the following lemma. We omit a proof here, but it can be found in, for instance [10] or [12].

Lemma 2.1.7. Let $f$ be a distribution, and $\phi \in C_{0}^{\infty}(X)$. Then $\Sigma(\phi f) \subseteq \Sigma(f)$.

Now we are ready to define the wave front set of a distribution $f \in \mathcal{D}^{\prime}(X)$. As in the case of the singular support and frequency set, it is easier to define the complement. We say $\left(x_{0}, \xi_{0}\right) \in X \times \mathbb{R}^{n} \backslash\{0\}$ is not in the wave front set of $f$ (denoted by $W F(f)$ ) if there 
exists a smooth, compactly supported function $\phi \in C_{0}^{\infty}(X)$ with $\phi\left(x_{0}\right) \neq 0$, and an open cone $\Gamma \subset \mathbb{R}_{\xi}^{n} \backslash\{0\}$ containing $\xi_{0}$ so that

$$
|\widehat{\phi f}(\xi)| \leq C_{N}(1+|\xi|)^{-N}
$$

for all $N$ positive, for every $\xi \in \Gamma$, and for some constant $C_{N}$.

Thus, the wave front set of a distribution generalizes the notion of singular support, in the following sense:

Proposition 2.1.8. Let $\pi$ be the natural projection from $X \times \mathbb{R}^{n} \backslash\{0\} \rightarrow X$. Then $\pi(W F(f))=\operatorname{sing} \operatorname{supp}(f)$.

Proof. Suppose $x_{0} \notin \operatorname{sing} \operatorname{supp}(f)$; then there is a $\phi \in C_{0}^{\infty}(X)$ so that $\phi\left(x_{0}\right) \neq 0$ and

$$
|\widehat{\phi f}(\xi)| \leq C_{N}(1+|\xi|)^{-N}
$$

for all positive $N$ and every $\xi$, for some constant $C_{N}$, and thus $\left(x_{0}, \xi\right) \notin W F(f)$ for all $\xi \neq 0$.

Now, suppose $x_{0} \notin \pi(W F(f))$. Using compactness of $\mathbb{S}^{n-1}$, we can find functions $\phi_{k} \in C_{0}^{\infty}(X)$ with $\phi_{k}\left(x_{0}\right) \neq 0$ and associated cones $\Gamma_{k}$ for $k=1,2, \ldots, K$ such that $\cup_{k=1}^{K} \Gamma_{k} \supset \mathbb{R}_{\xi}^{n} \backslash\{0\}$ and such that, for every positive $N$, there exists $C_{N, k}$ such that

$$
\widehat{\phi_{k} f}(\xi) \leq C_{N, k}(1+|\xi|)^{-N}
$$

for all $\xi \in \Gamma_{k}$. That is, $\Gamma_{k} \cap \Sigma\left(\phi_{k} f\right)=\emptyset$. Then, if $\phi=\Pi_{k=1}^{K} \phi_{k}$, we have $\phi \in C_{0}^{\infty}(X)$ with $\phi\left(x_{0}\right) \neq 0$, and by our previous lemma,

$$
\Sigma(\phi f) \subseteq \cap_{k=1}^{K} \Sigma\left(\phi_{k} f\right)=\emptyset
$$

From this, and the definition of $\Sigma$, it is now clear that $x \notin \operatorname{sing} \operatorname{supp}(f)$, and the proof is complete.

\subsubsection{The Hamiltonian and propagation of singularities}

Having now concluded our introduction to the wave front set, we want to explore the effect of a Fourier integral operator on the wave front set of a distribution. In short, if $P$ is an F.I.O. and $f$ is a distribution, we want to know how $W F(f)$ and $W F(P f)$ are related. 
We start by recalling that for a differential operator $P$, the following condition holds

$$
\operatorname{supp}(P u) \subset \operatorname{supp}(u)
$$

for all $u$, known as the local property. In general, a pseudodifferential operator (let alone an F.I.O.) will not satisfy the local property, but the following analog is available:

Theorem 2.1.9 (Pseudolocal Property). If $P$ is a pseudodifferential operator (of any order) and $u \in \mathcal{E}^{\prime}(X)$ then

$$
\operatorname{sing} \operatorname{supp}(P u) \subset \operatorname{sing} \operatorname{supp}(u) \text {. }
$$

We will prove this theorem shortly, following the method outlined in [38] (see also [14], [30], etc.). The key idea is to associate $P$ with its Schwartz kernel $k(x, y) \in \mathcal{D}^{\prime}(X \times X)$, and then establish the following lemma. We recall that the relationship between an operator $P$ and its Schwartz kernel is given by

$$
\langle P u, v\rangle=\langle k, u(x) v(y)\rangle .
$$

Lemma 2.1.10. Suppose $k \in \mathcal{D}^{\prime}(X \times X)$ is the Schwartz kernel of a continous map $P$ : $C_{0}^{\infty}(X) \rightarrow C^{\infty}(X)$ with a continuous extension $P: \mathcal{E}^{\prime}(X) \rightarrow \mathcal{D}^{\prime}(X)$ and $k$ is $C^{\infty}$ away from the diagonal in $X \times X$. Then

$$
\operatorname{sing} \operatorname{supp}(P u) \subset \operatorname{sing} \operatorname{supp}(u)
$$

for $u \in \mathcal{E}^{\prime}(X)$.

Proof. Choose $x_{0} \notin \operatorname{sing} \operatorname{supp}(u)$. Then, we can find $\phi \in C_{0}^{\infty}(X)$ so that $\phi=1$ in a neighborhood $U$ of $x$, and $\phi u \in C_{0}^{\infty}$. Next, choose $\psi \in C_{0}^{\infty}(X)$ supported in $U$, with $\psi\left(x_{0}\right) \neq 0$. Then, we have

$$
\psi P u=\psi P \phi u+\psi P(1-\phi) u .
$$


Because $\psi, \phi u \in C_{0}^{\infty}$ and $P: C_{0}^{\infty} \rightarrow C^{\infty}$, we have $\psi P \phi u \in C^{\infty}$. Next, because the supports of $\psi$ and $1-\phi$ are disjoint, there is an $\epsilon>0$ so that $\psi(x) k(x, y)(1-\phi)(y)=0$ for $|x-y|<\epsilon$. Thus,

$$
\begin{aligned}
\psi P(1-\phi) u & =\psi(x) \int k(x, y)(1-\phi)(y) u(y) d y \\
& =\int_{|x-y|<\epsilon} \psi(x) k(x, y)(1-\phi)(y) u(y) d y+\int_{|x-y| \geq \epsilon} \psi(x) k(x, y)(1-\phi)(y) u(y) d y \\
& =\int_{|x-y| \geq \epsilon} \psi(x) k(x, y)(1-\phi)(y) u(y) d y
\end{aligned}
$$

which defines an integral operator with smooth kernel $\chi_{|x-y| \geq \epsilon} \psi(x) k(x, y)(1-\phi)(y)$. Thus $\psi P(1-\phi) u \in C^{\infty}$, and combined with our earlier observation, we have that $x_{0} \notin \operatorname{sing} \operatorname{supp}(P u)$

As we noted following Theorem 2.1.5 pseudodifferential operators are always continuous as maps $C_{0}^{\infty} \rightarrow C^{\infty}$ and have continuous extensions $\mathcal{E}^{\prime} \rightarrow \mathcal{D}^{\prime}$. Thus to prove Theorem 2.1.9, it just remains to prove the integral kernel of a pseudodifferential operator is smooth away from the diagonal. To do this, we relate the kernel and the symbol of $P$

$$
\begin{aligned}
\langle k, u \otimes v\rangle & =\langle P u, v\rangle \\
& =\int(P u)(x) v(x) d x \\
& =\iiint e^{i\langle x-y, \xi\rangle} p(x, \xi) u(y) d y d \xi v(x) d x \\
& =\iint\left(\int e^{i\langle x-y, \xi\rangle} p(x, \xi) d \xi\right) u(y) v(x) d x d y
\end{aligned}
$$

so that $k(x, y)=\int e^{i\langle x-y, \xi\rangle} p(x, \xi) d \xi$. Then

$$
\begin{aligned}
(x-y)^{\alpha} k(x, y) & =\int(x-y)^{\alpha} e^{i\langle x-y, \xi\rangle} p(x, \xi) d \xi \\
& =\int D_{\xi}^{\alpha} e^{i\langle x-y, \xi\rangle} p(x, \xi) d \xi \\
& =\int e^{i\langle x-y, \xi\rangle} D_{\xi}^{\alpha} p(x, \xi) d \xi
\end{aligned}
$$


which is absolutely convergent for $\alpha$ large enough (if $P$ is of order $m$, then $m-|\alpha|<-n$ is sufficient). Further, taking $j$ derivatives (and increasing the order of $\alpha$ so that $m-|\alpha|+j<$ $-n)$ still gives an absolutely convergent integral, showing $(x-y)^{\alpha} k \in C^{j}(X \times X)$. Thus, $k$ is smooth off the diagonal as claimed, and we have proved the theorem.

While the pseudolocal property is often highly useful, in some cases it fails to yield much information. For instance, if $P$ is a wave operator $\partial_{t}^{2}-\Delta$ and $u$ is a solution $P u=0$, the pseudolocal property only states

$$
\emptyset=\operatorname{sing} \operatorname{supp}(0)=\operatorname{sing} \operatorname{supp}(P u) \subset \operatorname{sing} \operatorname{supp}(u)
$$

which tells us nothing about the singularities of $u$ (or anything else, for that matter!). To obtain a suitable description of $\operatorname{sing} \operatorname{supp}(u)$, we develop some geometric ideas. We first briefly recall the notion of the tangent bundle and cotangent bundle of $X$. Again, we restrict our attention to open sets of $\mathbb{R}^{n}$, but in general, $X$ can be a manifold.

If $x_{0}$ is a point in $X$, and $T_{x_{0}} X$ is the collection of tangent vectors to $X$ at $x_{0}$, we denote the tangent bundle of $X$ by $T X=\cup_{x \in X} T_{x} X \simeq X \times \mathbb{R}^{n}$. Next, if $T_{x_{0}}^{*} X$ is the dual space of $T_{x_{0}} X$, we call $T^{*} X=\cup_{x \in X} T_{x}^{*} X \simeq X \times \mathbb{R}^{n}$ the cotangent bundle of $X$. If $\pi: T X \rightarrow X$ is the natural projection, we call a smooth map $V: X \rightarrow T X$ such that $\pi \circ V$ is the identity (i.e. a smooth section of $T X$ ) a vector field, and define a covector field similarly. We call the collection of all such maps $\Gamma(T X)$ and $\Gamma\left(T^{*} X\right)$ respectively. Finally, note that, since $T X$ and $T^{*} X$ are both open sets in $\mathbb{R}^{2 n}$, we can repeat these constructions to define $T\left(T^{*} X\right), T^{*}\left(T^{*} X\right)$, etc.

If $\left(x_{1}, \ldots, x_{n}\right)$ are coordinates for $X$, then $\frac{\partial}{\partial x_{1}}, \ldots, \frac{\partial}{\partial x_{n}}$ is a basis for $T X$, in the sense that, for any smooth vector field $V: X \rightarrow T X, V$ can be written

$$
V=\sum_{j=1}^{n} v_{j}(x) \frac{\partial}{\partial x_{j}}
$$

for some smooth functions $v_{j}$. Similarly, $d x_{1}, \ldots, d x_{n}$ form a basis for $T^{*} X$. We also use the notation $(x, \xi)=\left(x_{1}, \ldots, x_{n}, \xi_{1}, \ldots, \xi_{n}\right)$ to describe a general point in $T^{*} X$.

If $p(x, \xi)$ is a smooth function on $T^{*} X$, we can define a vector field $H_{p} \in \Gamma\left(T\left(T^{*} X\right)\right)$ by

$$
H_{p}=\sum_{j=1}^{n} \frac{\partial p}{\partial \xi_{j}} \frac{\partial}{\partial x_{j}}-\frac{\partial p}{\partial x_{j}} \frac{\partial}{\partial \xi_{j}} .
$$


This vector field is called the Hamiltonian vector field of $p$.

Recall that an integral curve of a vector field $V \in \Gamma(T X)$ is a path $\gamma:[a, b] \rightarrow X$ such that $\frac{d}{d s} \gamma(s)=V(\gamma(s))$ for all $s \in[a, b]$. With this in mind, we define a bicharacteristic of $p$ as an integral curve of $H_{p}$; i.e. it is a solution $(x(s), \xi(s))$ of

$$
\begin{aligned}
& \dot{x}_{j}=\frac{\partial p}{\partial \xi_{j}} \\
& \dot{\xi}_{j}=-\frac{\partial p}{\partial x_{j}}
\end{aligned}
$$

(where $\dot{x}$ is shorthand for $\frac{d}{d s} x$, etc.). Additionally, if $p$ vanishes along such an integral curve, (i.e. $p(x(s), \xi(s))=0$ for all $s$ ), then we call the curve a null bicharacteristic. The projection of a null bicharacteristic to $X$ (i.e. $x(s)$ ) is called a ray.

We note here that, by construction $H_{p} p=0$, and so $p$ is constant along the integral curves of $H_{p}$. That is, to check whether or not a bicharacteristic is null, it is sufficient to check $p(x(0), \xi(0))=0$.

As an example, for the function $p=c^{2}|\xi|^{2}-\tau^{2}$ (which is the principal symbol of the differential operator $\left.\partial_{t}^{2}-c^{2} \Delta\right)$, the Hamiltonian is

$$
\frac{1}{2} H_{p}=c^{2} \sum_{j=1}^{n} \xi_{j} \frac{\partial}{\partial x_{j}}-\tau \frac{\partial}{\partial t} .
$$

Moving forward, we omit the factor of 2 (as it yields the same solution, up to a reparameterization) in $H_{p}$ and note that its integral curves $\gamma: s \mapsto(x(s), t(s), \xi(s), \tau(s))$ will satisfy

$$
\begin{aligned}
\dot{x}_{j} & =c^{2} \xi_{j}, \\
\dot{t} & =-\tau, \\
\dot{\xi}_{j} & =0, \\
\dot{\tau} & =0 .
\end{aligned}
$$

Suppose $0 \in[a, b]$, and $\gamma(0)=\left(x_{0}, 0, \xi_{0}, \tau_{0}\right)$. Then a solution is given by

$$
\gamma(s)=\left(x_{0}+c^{2} \xi_{0} s,-\tau_{0} s, \xi_{0}, \tau_{0}\right) .
$$


If $p(\gamma(0))=0$, then we have $\tau_{0}= \pm c\left|\xi_{0}\right|$, and if we reparamaterize $\gamma$ in terms of $t$, we have bicharacteristics

$$
\gamma(t)=\left(x_{0} \pm c \frac{\xi_{0}}{\left|\xi_{0}\right|} t, t, \xi_{0}, \tau_{0}\right)
$$

The corresponding ray is then given by $\left(x_{0} \pm c \frac{\xi_{0}}{\left|\xi_{0}\right|} t, t\right)$ (or, in view of our reparameterization, simply $\left.x_{0} \pm c \frac{\xi_{0}}{\left|\xi_{0}\right|} t\right)$.

Now we seek an exact description of how the singularities of a wave's initial data influence the singularities of the solution. A proof of this theorem can be found in any of the references given for this section.

Theorem 2.1.11. Let $P$ be a pseudodifferential operator of order $m$, with real-valued principal symbol $p(x, \xi)$ positively homogeneous of degree $m$ in $\xi$. If $\gamma:[a, b] \rightarrow T^{*} X$ is a null bicharacteristic of $p$ and $u$ satisfies $P u=0$, then either $\gamma([a, b]) \subset W F(u)$ or $\gamma([a, b]) \cap W F(u)=\emptyset$.

Thus, if $u$ is a solution of a wave equation, with initial displacement $f$ (and zero initial velocity), we can describe precisely where $u$ is singular: $u$ is singular at $(t, x)$ if and only if there is $\left(x_{0}, \xi_{0}\right) \in W F(f)$ such that the projection of a null bicharacteristic with $\gamma(0)=$ $\left(x_{0}, 0, \xi_{0}, \tau_{0}\right)$ to $\mathbb{R} \times X$ passes through $(t, x)$. Or, in briefer terms, singularities will propagate along the null bicharacteristics/rays of $p$.

\subsubsection{The non-trapping hypothesis}

Now we are in a position to better discuss the non-trapping hypothesis introduced in the previous chapter. Let $X=\mathbb{R} \times \mathbb{R}^{n}, P=\partial_{t}^{2}-c^{2}(x) \Delta$ be a wave operator with principal symbol $p$, and let $\Omega$ be a bounded open set in $\mathbb{R}^{n}$. As in our earlier example, we can reparameterize each null bicharacteristic of $p$ in terms of $t$, and so we think of them as maps $\gamma: t \mapsto(x(t), \xi(t))$.

Now, we consider the set of all null bicharacteristics $\gamma$ with $\gamma(0)=\left(x_{0}, \xi_{0}\right)$ such that $x_{0} \in \Omega$ (and of course $\xi_{0} \neq 0$ ). We say that $(\Omega, c)$ is non-trapping if each of the correpsonding rays leaves $\Omega$ in finite time, and the supremum of these times is finite as well. For a nontrapping $(\Omega, c)$, we will call the supremum $T(\Omega)$. 


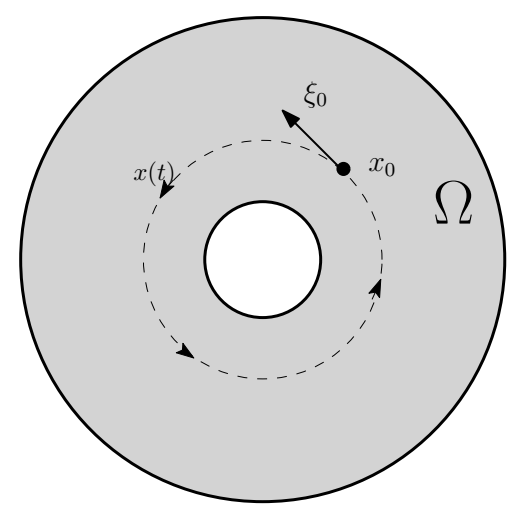

Figure 2.1: An example of a trapped geodesic.

One may gain additional understanding of the nature of this definition by considering an example of a trapping metric: consider $c(x)=|x|$, with $\Omega$ an annulus $\left\{x: r_{1}<|x|<r_{2}\right\}$. Then, for $x_{0} \in \Omega$, if $\xi_{0}$ is perpendicular to $x_{0}$, it is straightforward to check that the resulting ray is $\left\{x:|x|=\left|x_{0}\right|\right\}$ (see Figure 2.1), which will remain inside $\Omega$ for all time.

Because singularities propagate along bicharacteristics, as we discussed in the previous section, the non-trapping hypothesis also ensures that any singularities of $f$ will have left $\Omega$ by $T(\Omega)$, or put another way, that $u(t, x)$ is a smooth function on $\Omega$ for $t>T(\Omega)$.

\section{$2.2 \quad$ Functional Analysis}

In the proof of our main result, it will be important to determine when, for a bounded, linear operator $K,\|K f\|<\|f\|$ for all $f$ implies $\|K\|<1$. To describe the correct class of operators for which this statement is true, we will need some preliminaries from functional analysis; in particular, we will discuss the spectrum of a linear operator, compact operators, and the spectral properties of compact operators. The results stated in this section can be found in any textbook on functional analysis; see e.g. [28] or [29], etc.

\subsubsection{The spectrum of a bounded linear operator}

Let $X$ be a Banach space, $T: X \rightarrow X$ a bounded, linear operator, and $\lambda$ a complex number. If $T-\lambda I$ has a bounded, linear inverse, we say $\lambda$ is in the resolvent set of $T$. Otherwise, 
we say $\lambda$ is in the spectrum of $T$, or $\lambda \in \sigma(T)$.

Determining whether or not $T-\lambda I$ has an inverse is made easier by the following key result from functional analysis.

Theorem 2.2.1 (Open Mapping Theorem). Let $X$ and $Y$ be Banach spaces, and $T: X \rightarrow Y$ be a bounded linear operator. If $T$ is surjective, then $T(E)$ is open in $Y$ for every open set $E \subset X$.

As a consequence of the open mapping theorem, $T-\lambda I$ will have a bounded linear inverse precisely when it is bijective - because it is injective, the map $(T-\lambda I)^{-1}$ is well defined, and because it is surjective, the inverse is continuous. Thus, when determining whether or not a value $\lambda$ is in the spectrum of $T$, it is sufficient to check the bijectivity of $T-\lambda I$.

Of course, bijectivity can fail in a number of ways. First, if there is a non-zero vector $x \in X$ so that $(T-\lambda I) x=0$, then clearly $T$ is not injective, and so not invertible. In this case, we call $\lambda$ an eigenvalue, $x$ an eigenvector, and the collection of all eigenvalues the point spectrum of $T$. In the case of a finite dimensional vector space $V$, because of the rank-nullity theorem, an operator $T: V \rightarrow V$ fails to be invertible precisely when it fails to be injective, and so the spectrum consists entirely of eigenvalues.

For infinite dimensional Banach spaces, the situation is not so straightforward, and there are other ways in which $T-\lambda I$ can fail to be bijective. In particular, if $T-\lambda I$ is not bounded below, or does not have dense range, then it is not bijective.

In the first case, exhibiting a sequence $\left\{x_{n}\right\} \subset X$ with $\left\|x_{n}\right\|=1$ and $\left\|T x_{n}-\lambda x_{n}\right\| \rightarrow 0$ is equivalent to showing $T-\lambda I$ fails to be bounded below. We call such values of $\lambda$ the approximate point spectrum. Of course, if $\lambda$ is an eigenvalue, and $x$ is a corresponding eigenvector, then the constant sequence $x_{n}=x /\|x\|$ is such a sequence. Thus, the point spectrum is always a subset of the approximate point spectrum.

There can be values of $\lambda$ which lie in the approximate point spectrum, but not the point spectrum. Let $X=\ell^{2}(\mathbb{Z})$, the space of bi-infinite sequences $x(k)$ which are squaresummable, i.e. $\sum_{k=-\infty}^{\infty}|x(k)|^{2}<\infty$. Let $T$ be the left-shift operator on this space; explic- 
itly,

$$
T x(k)=x(k+1) .
$$

We will show that $\lambda=1$ is in the approximate point spectrum, but is not an eigenvalue. To do this, we define a sequence $x_{n}$ by

$$
x_{n}(k)= \begin{cases}0 & : k \leq 0, \\ \frac{1}{\sqrt{n}} & : 1 \leq k \leq n, \\ 0 & : k>n .\end{cases}
$$

Clearly, $\left\|x_{n}\right\|=1$, and further

$$
T x_{n}(k)-x_{n}(k)= \begin{cases}\frac{1}{\sqrt{n}} & : k=0 \\ \frac{-1}{\sqrt{n}} & : k=n \\ 0 & : \text { otherwise }\end{cases}
$$

so that $\left\|T x_{n}-x_{n}\right\|=\frac{\sqrt{2}}{\sqrt{n}}$ which tends to zero as $n \rightarrow \infty$. Thus, $\lambda=1$ is in the approximate spectrum. On the other hand, if $\lambda=1$ is an eigenvalue, then there is a non-zero sequence $x(k)$ so that $T x=x$. This would imply that $x(k)=x(k+1)$ for all $k$, and so $x$ is constant. But such a sequence is either zero, or it is not square-summable, and so 1 is not an eigenvalue.

Finally, if $\lambda$ is such that $T-\lambda I$ is injective, but fails to have dense range, we say that $\lambda$ is in the residual spectrum of $T$. To demonstrate this, we let $X=\ell^{2}(\mathbb{N})$ and $T$ be the right shift operator

$$
T x(k)= \begin{cases}0 & : k=0, \\ x(k-1) & : k>0,\end{cases}
$$

and show that $\lambda=0$ is in the residual spectrum (i.e. that $T$ is injective, but does not have dense range). It is easy to see that $\|T x\|=\|x\|$ for every $x$, so $T$ is injective and bounded below, and so $\lambda=0$ is not part of the approximate spectrum. But $x=(1,0,0, \ldots, 0, \ldots)$ is clearly not in the range of $T$, and so $T$ fails to be bijective. Thus, the claim is proven. 
At this point we note that it is possible for a value $\lambda \in \sigma(T)$ to lie in both the approximate and residual spectrum, if $T-\lambda I$ is injective, not bounded below, and does not have dense range. In view of this, we call the continuous spectrum of $T$ the values of $\lambda$ which are in the approximate spectrum, but not the residual spectrum or the point spectrum (i.e., those values of $\lambda$ for which $T-\lambda I$ is injective, has dense range, but is not bounded below).

\subsubsection{Compact operators}

In this section, we seek to answer our question: "If $X$ and $Y$ are Banach spaces, $T: X \rightarrow Y$ is a bounded linear operator, and $\|T x\|_{Y}<\|x\|_{X}$ for all $x \in X$, is it true that $\|T\|<1$ ?". Here, $\|T\|$ indicates the standard operator norm

$$
\|T\|=\sup _{x \in X:\|x\|=1}\|T x\| .
$$

Of course, if $X$ is finite dimensional, this is true; if $\|T\|=1$, there is an $x$ so that this supremum is attained. In general, however, this does not hold, as the unit ball in an infinite dimensional vector space is not compact. For example, take $X$ to be $L^{2}([0,1])$ and let $T: X \rightarrow X$ be defined by

$$
T: f(t) \mapsto t f(t)
$$

It is not hard to see $\|T f\|<\|f\|$ for all $f$. However, if we consider the sequence of functions $f_{n}=\sqrt{n} \chi_{[1-1 / n, 1]}$, then $\left\|f_{n}\right\|=1$ for all $n$, but

$$
\left\|t f_{n}\right\|=\left(n \int_{1-1 / n}^{1} t^{2} d t\right)^{1 / 2}=\sqrt{1-\frac{1}{n}+\frac{1}{3 n^{2}}} \rightarrow 1
$$

as $n \rightarrow \infty$. Thus, $\|T\|=1$.

Thus, we seek to characterize operators that behave in some way like operators over finite dimensional spaces. As it turns out, the correct description is that of compactness: we say a bounded linear operator $K: X \rightarrow Y$ is compact if the image of the unit ball $\{x \in X:\|x\| \leq 1\}$ is pre-compact in $Y$.

Equivalently, we can say $K$ is compact if and only if the image of any bounded set is pre-compact, and if and only if, for every bounded sequence $\left\{x_{n}\right\} \subset X,\left\{K x_{n}\right\} \subset Y$ has a convergent subsequence. 
It is clear that, if $X$ and $Y$ are finite dimensional, then every linear operator mapping $X$ to $Y$ is compact. Further, if $K: X \rightarrow Y$ has finite dimensional range, then it is compact. To see this, we suppose $T x=\sum_{k=1}^{K} a_{k} y_{k}$ for some $K$ vectors $y_{k} \in Y$. Then, if $x_{n}$ is a bounded sequence in $X$, each of the $K$ sequences (in $n$ ) of coefficients $a_{k, n}$ is bounded, and so has a convergent subsequence. By selecting futher subsequences, we can find a convergent subsequence of $T x_{n}$.

Further, compositions of bounded operators with compact operators remain compact:

Proposition 2.2.2. If $K$ is compact, and $T$ is a bounded linear operator, then $T K$ and KT are compact.

Proof. First, let $T: X \rightarrow Y$ and $K: Y \rightarrow Z$, and let $x_{n}$ be a bounded sequence in $X$; we will show $K T x_{n}$ has a convergent subsequence. Because $T$ is bounded, $T x_{n}$ is a bounded sequence in $Y$. Then, because $K$ is compact, $K\left(T x_{n}\right)$ will have a convergent subsequence, showing $K T$ is compact.

Next, suppose $K: X \rightarrow Y$ and $T: Y \rightarrow Z$. We will show that the image of the unit ball $U_{1}$ in $X$ under $T K$ is pre-compact. Indeed, because $K$ is compact, $K\left(U_{1}\right)$ is compact, and so $T K\left(U_{1}\right)$ is compact as the continuous image of a compact set.

Another important class of examples are smoothing operators; recall that a bounded linear operator $K$ is called smoothing if it can be continuously extended to a map $K$ : $\mathcal{E}^{\prime}(Y) \rightarrow C^{\infty}(X)$. By the Schwartz kernel theorem, $K$ has a smooth Schwartz kernel $k$, i.e. there exists $k \in C^{\infty}(X \times Y)$ such that

$$
K f(x)=\int k(x, y) f(y) d y
$$

for all $f$. With this integral representation, we can prove that some smoothing operators are compact, provided the spaces $X$ and $Y$ are compact.

Proposition 2.2.3. Let $\Omega$ be a bounded domain in $\mathbb{R}^{n}$, and suppose $K: H_{0}^{1}(\bar{\Omega}) \rightarrow H_{0}^{1}(\bar{\Omega})$ is smoothing. Then $K$ is compact.

Proof. As a map continuously extended to $\mathcal{E}^{\prime}(\bar{\Omega}) \rightarrow C^{\infty}(\bar{\Omega}), K$ has a smooth integral kernel $k$. Further, $k$ is uniformly continuous (as it is smooth on the compact set $\bar{\Omega} \times \bar{\Omega}$ ). 
Now we use the Arzelà-Ascoli theorem; we need to show that if $\left\{f_{n}\right\} \subset H_{0}^{1}(\bar{\Omega})$ with $\left\|f_{n}\right\| \leq 1$ (here, $\|\cdot\|$ indicates the $H_{0}^{1}$ norm), then $\left\{K f_{n}\right\}$ is uniformly bounded and equicontinuous.

Indeed, the uniform boundedness follows from the boundedness of $K$ :

$$
\left\|K f_{n}\right\| \leq\|K\|\left\|f_{n}\right\| \leq\|K\|
$$

and so the functions are uniformly bounded by $\|K\|$.

Next, to demonstrate equicontinuity, we use the uniform continuity of the integral kernel $k$. If $\epsilon>0$, then there is $\delta>0$ so that $\left|k(x, y)-k\left(x_{0}, y\right)\right| \sqrt{\operatorname{vol}(\Omega)}<\epsilon$ whenever $\left|x-x_{0}\right|<$ $\delta$. Thus, for any $n$,

$$
\left|K f_{n}(x)-K f_{n}\left(x_{0}\right)\right| \leq \int\left|k(x, y)-k\left(x_{0}, y\right)\right|\left|f_{n}(y)\right| d y<\epsilon\left\|f_{n}\right\|_{L^{2}(\bar{\Omega})} \leq \epsilon\left\|f_{n}\right\| \leq \epsilon
$$

whenever $\left|x-x_{0}\right|<\delta$, and thus all functions $K f_{n}$ are equicontinuous.

Compact operators have many nice properties, and in some ways generalize the idea of finite dimensional operators. In particular, there is a very nice characterization of their spectrum.

Theorem 2.2.4 (Fredholm Alternative). Let $H$ be a separable Hilbert space, and $K: H \rightarrow$ $H$ a compact operator. Then the spectrum of $K$ is a set of discrete points whose only possible limit point is 0 . Furthermore, for every non-zero $\lambda \in \sigma(K), \lambda$ is an eigenvalue, and $\operatorname{ker}(K-\lambda I)$ is finite-dimensional.

Further, for compact operators that are also self-adjoint, the eigenfunctions form an orthogonal basis of the space, much like the finite dimensional case.

Theorem 2.2.5 (Hilbert-Schmidt). Let A be a compact, self-adjoint operator on a separable Hilbert space $H$. Then there is a complete, orthonormal basis $\left\{\phi_{n}\right\}$ such that $A \phi_{n}=\lambda_{n}$ with $\lambda_{n} \rightarrow 0$ as $n \rightarrow \infty$.

This allows us to prove the following infinite dimensional version of the Rayleigh quotient. 
Theorem 2.2.6 (Rayleigh Quotient). Let $H$ be a separable Hilbert space, A a compact, self-adjoint operator on $H$, and let $\lambda_{1}$ be the largest positive eigenvalue of $A$. Then

$$
\sup _{\|f\|=1}\langle A f, f\rangle=\lambda_{1}
$$

Proof. Let $\left\{\phi_{n}\right\}$ be a basis of $H$ consisting of eigenvectors of $A$, as in the previous theorem. Choose any $f \in H$, with $\|f\|=1$. We can write $f=\sum_{n=1}^{\infty}\left\langle f, \phi_{n}\right\rangle \phi_{n}$ so that $\|f\|^{2}=$ $\sum_{n=1}^{\infty}\left\langle f, \phi_{n}\right\rangle^{2}=1$. Next, observe

$$
\langle A f, f\rangle=\sum_{n=1}^{\infty} \lambda_{n}\left\langle f, \phi_{n}\right\rangle^{2} \leq \lambda_{1} .
$$

Thus, $\langle A f, f\rangle \leq \lambda_{1}$ for all $f$ with norm 1 . Finally, if $f=\phi_{1}$, equality is attained, and the result is proved.

Finally, we are in a position to prove the main result of this section, with which we conclude this chapter.

Proposition 2.2.7. If $H$ is a separable Hilbert space, and $K: H \rightarrow H$ is a compact operator, and $\|K f\|<\|f\|$ for all $f \in H$, then $\|K\|<1$.

Proof. Suppose $\|K\|=1$, i.e. $\sup _{\|f\|=1}\|K f\|=1$. Then

$$
\sup _{\|f\|=1}\left\langle K^{*} K f, f\right\rangle=\sup _{\|f\|=1}\langle K f, K f\rangle=\sup _{\|f\|=1}\|K f\|^{2}=1 .
$$

Then, by the preceding theorem, since $K^{*} K$ is a compact, self-adjoint operator, $\lambda=1$ is an eigenvalue of $K^{*} K$, and so there is $g \in H$ so that $K^{*} K g=g$. But $\|K g\|^{2}=\langle K g, K g\rangle=$ $\left\langle K^{*} K g, g\right\rangle=\langle g, g\rangle=\|g\|^{2}$, which is a contradiction. 
Chapter 3

\section{THERMOACOUSTIC TOMOGRAPHY IN ELASTIC MEDIA}

Having now covered the necessary preliminary mathematics, we seek to prove the main result of this work. We begin by discussing the basic properties of elastic waves, including especially unique continuation and energy of solutions, and then move to the reconstruction process.

\subsection{The Elastic Wave Equation}

The elastic wave equation, in its most general form is given by

$$
\rho \frac{\partial^{2}}{\partial t^{2}} u_{i}=\sum_{j, k, l=1}^{n} \frac{\partial}{\partial x_{j}} c_{i j k l} \frac{\partial}{\partial x_{k}} u_{l}
$$

where $\rho$ is the density, and $c_{i j k l}$ is the elastic tensor. We will find it convenient to take $\rho=1$ from this point on, as it simplifies the analysis, and does not otherwise adversely affect our results.

While a priori, the elastic tensor has $n^{4}$ entries, there are many symmetries that can be exploited. In particular, $c_{i j k l}=c_{j i k l}=c_{i j l k}=c_{i k j l}$ in the general case, and for particular types of media (e.g. isotropic, orthotropic, etc.), further simplifications are possible.

We will focus primarily on isotropic media. Physically, an isotropic medium responds the same in every direction, and the wave equation is given by

$$
\frac{\partial^{2}}{\partial t^{2}} u=\nabla \cdot\left(\mu(x)\left((\nabla u)+(\nabla u)^{T}\right)\right)+\nabla(\lambda(x) \nabla \cdot u) .
$$

The right-hand-side has principal part

$$
\begin{aligned}
& \mu(x) \nabla \cdot\left((\nabla u)+(\nabla u)^{T}\right)+\lambda(x) \nabla \nabla \cdot u \\
& =\mu \Delta u+\mu \nabla \nabla \cdot u+\lambda \nabla \nabla \cdot u \\
& =(\lambda+2 \mu) \nabla \nabla \cdot u+\mu(\Delta-\nabla \nabla \cdot) u,
\end{aligned}
$$


with principal symbol (ignoring the sign change)

$$
p(x, \xi)=(\lambda+2 \mu) \xi \xi^{T}+\mu\left(|\xi|^{2} I-\xi \xi^{T}\right),
$$

a symmetric matrix-valued symbol with (for fixed $x, \xi$ ) eigenvector $\xi$ corresponding to eigenvalue $(\lambda+2 \mu)|\xi|^{2}$ (of multiplicity 1) and eigenvectors $\xi_{2}, \ldots, \xi_{n} \in \xi^{\perp}$ corresponding to eigenvalue $\mu|\xi|^{2}$ (of multiplicity $n-1$ ). Because $p$ is symmetric, it is always possible to choose these eigenvectors so that they are orthogonal. We note here that, for the general anisotropic elastic system, the eigenvalues are more complicated functions of $x$ and $\xi$, and can even change multiplicity, though we will not discuss this further here.

To study the microlocal behavior of this system, we will seek to diagonalize this system into blocks corresponding to these eigenvalues, effectively decoupling the wave into its component modes, and then analyze each mode individually. We call the modes corresponding to the eigenvalue $\lambda+2 \mu$ the $\mathrm{P}$-modes, and the modes for $\mu$ the $\mathrm{S}$-modes.

In the case of homogeneous media (constant $\lambda$ and $\mu$ ), this decoupling is complete. Here, we demonstrate this by constructing projection operators $\Pi_{P}$ and $\Pi_{S}$

$$
\begin{aligned}
& \Pi_{P}=\Delta^{-1} \nabla \nabla \cdot \\
& \Pi_{S}=I-\Pi_{S}=\Delta^{-1}(\Delta-\nabla \nabla \cdot)=\Delta^{-1}(-\nabla \times \nabla \times)
\end{aligned}
$$

so that

$$
P=(\lambda+2 \mu) \Delta \Pi_{P}+\mu \Delta \Pi_{S}
$$

and so that if we then write $u=\Pi_{P} u+\Pi_{S} u=u_{P}+u_{S}$ then

$$
\begin{aligned}
& P u_{P}=(\lambda+2 \mu) \Delta u_{P} \\
& P u_{S}=\mu \Delta u_{S} .
\end{aligned}
$$

In the case of non-homogeneous isotropic media, we can carry out the same construction, though the identity above only holds modulo lower order terms, i.e.

$$
P=(\lambda+2 \mu) \Delta \Pi_{P}+\mu \Delta \Pi_{S}+\text { l.o.t. }
$$


Despite this minor setback, diagonalization on the level of the principal symbol still enables us to more easily study the operator, and deduce information about microlocal properties, such as propagation of singularities. In fact, in the work of Taylor (see [38], [39], see also Stolk [33]), it is shown that there is a matrix-valued pseudo-differential operator $Q$ of order 0 such that $Q^{-1} P Q$ is block-diagonal modulo a smoothing term, each block corresponding to an eigenvalue (i.e. to the wave modes). In practical terms, we can take the principal symbol of $Q$ to be a matrix-valued symbol $q(x, \xi)$ whose columns are the normalized eigenvectors of $p(x, \xi)$ (and so we can choose $q$ so that it is orthogonal).

We write our block-diagonal decomposition

$$
Q^{-1} P Q=\operatorname{diag}\left(P_{P}, P_{S}\right)
$$

and

$$
u_{M}=\sum_{i} Q_{M i}^{-1} u_{i}
$$

where $M=\mathrm{P}$ or $\mathrm{S}$ (here, the indices within the blocks have been suppressed) so that, for each mode (up to smoothing terms), we have

$$
\frac{\partial^{2}}{\partial t^{2}} u_{M}=P_{M} u_{M}
$$

Further, $P_{P}$ is a $1 \times 1$ block, and since each component of $u_{S}$ satisfies the same partial differential equation $\frac{\partial^{2}}{\partial t^{2}} u_{S, j}=\mu(x) \Delta u_{S, j}$ (albeit with different initial data), we study both

$$
\begin{aligned}
\frac{\partial^{2}}{\partial t^{2}} u_{P} & =(\lambda+2 \mu)(x) \Delta u_{P} \\
\frac{\partial^{2}}{\partial t^{2}} u_{S} & =\mu(x) \Delta u_{S}
\end{aligned}
$$

as scalar-valued wave equations.

From this, and in view of the pseudo-local property (Theorem 2.1.9), we can study the propagation of singularities for the original system by using the Hamilton-Jacobi equations for the scalar equations above. In particular, we can define and assess non-trapping in essentially the same way. We need to choose $T(\Omega)$ large enough that all rays corresponding to both $\mathrm{P}$ and S-modes will have time to exit $\Omega$, but other than this, we still have the same results. In particular $u(t, \cdot)$ is smooth on $\bar{\Omega}$ for $t>T(\Omega)$. 


\subsection{Unique Continuation and Conditions on the Lamé Parameters}

As we saw in the previous section, elastic wave equations can be decoupled into various modes of propagation, which is one of the key attributes distinguishing them from scalar wave equations. In fact, these modes correspond to two different speeds of propagation, the so-called P- and S-waves or modes (for 'pressure' and 'shear'). In particular, these two speeds $c_{1}(x)=\sqrt{2 \mu+\lambda}$ and $c_{2}(x)=\sqrt{\mu}$ correspond, as we have seen, to the eigenvalues of the principal symbol of $P$ :

$$
p(x, \xi)=(\lambda+2 \mu)(x) \xi \xi^{T}+\mu(x)\left(|\xi|^{2} I-\xi \xi^{T}\right) .
$$

Moving forward, it will be useful to define scalar wave operators corresponding with these speeds, and so with $a_{1}=1 /(2 \mu+\lambda)$ and $a_{2}=1 / \mu$, we define $\square_{a_{j}}, j=1,2$ by

$$
\square_{a_{j}}:=a_{j} \partial_{t}^{2}-\Delta
$$

The property of finite speed of propagation for the elasticity system is essentially the same as the scalar case, as demonstrated by the following definition and theorem. We will say that $u$ has finite speed of propagation in $(0, T) \times B\left(x_{0}, \epsilon\right)$, with maximum speed $c>0$, if for any $t_{0} \in[0, T), u\left(t_{0}, \cdot\right)=u_{t}\left(t_{0}, \cdot\right)=0$ in $B\left(x_{0}, \epsilon\right)$ implies $u=0$ a.e. in the cone $\cup_{0<s<\epsilon / c} C_{s}$, where $C_{s}=\left\{t=t_{0}+s\right\} \times B\left(x_{0}, \epsilon-c s\right)$.

Theorem 3.2.1. Assume that $\mu, \lambda \in C^{2}\left(\mathbb{R}^{3}\right)$, and suppose $u \in H^{2}$ solves (1.1). Then, for any open ball $B\left(x_{0}, \epsilon\right) \subset \mathbb{R}^{3}$, u has finite propagation speed in $(0, T) \times B\left(x_{0}, \epsilon\right)$, with maximum speed $c=\sup _{x \in B\left(x_{0}, \epsilon\right)} \sqrt{2 \mu+\lambda}$.

For a proof, we direct the reader to [24]. With this result in mind, we will hereafter assume that there exist constants $c^{-}, c^{+}$so that

$$
\begin{array}{r}
c^{+}=\sup _{x \in \mathbb{R}^{3}} \sqrt{2 \mu+\lambda}<\infty, \\
c^{-}=\inf _{x \in \mathbb{R}^{3}} \sqrt{\mu}>0 .
\end{array}
$$

We will also assume that $(\Omega, P)$ is non-trapping, meaning (recalling our discussion in the last section, as well as that given on page 19) that, for both the P- and S-modes, every ray 
(i.e. the projection of every bicharacteristic starting in $\Omega$ ) leaves $\Omega$ in finite time, and that the supremum of these times, which we will again call $T(\Omega)$, is also finite.

The presence of two speeds of propagation causes some difficulty regarding questions of unique continuation. As we have seen, for a homogeneous medium (i.e., the Lamé parameters are constant), the system is diagonalizable, and the $\mathrm{P}$ - and S-modes are preserved throughout the wave's evolution, effectively reducing this problem to the scalar case. In the more general setting however, $\mathrm{P}$-waves may transmit or reflect as $\mathrm{S}$-waves (and vice-versa) at an interface, making the question of unique continuation more subtle. For instance, if the P-wave vanishes on the boundary of a set for all time, one cannot necessarily conclude it vanishes on the interior as well; it may simply be transmitting as an S-wave instead. The extent to which the two modes can be decoupled is useful for understanding the reflection and transmission of singularities, and has been studied using the pseudodifferential calculus (see [39] and [38] for smooth $\mu$ and $\lambda$, and most recently, [3] for $\mu, \lambda \in C^{1,1}$ ).

For unique continuation in the classical case (a scalar wave equation with smooth coefficients), this problem essentially reduces to Holmgren's theorem (see, for instance, [38]). In the case of non-smooth coefficients for a scalar wave equation, there are good results due to Tataru (see [35],[36]). For the static Lamé system (i.e. the elliptic system $P u=0$ ), Lin, Nakamura, Uhlmann and Wang have recently shown (in [23]) that there is a strong unique continuation principle; specifically, if $\lambda \in L^{\infty}$ and $\mu \in C^{0,1}$, in $\mathbb{R}^{n}$ with $n \geq 2$, if a solution is zero in a neighborhood of any point, it is identically zero.

For the isotropic elastic system, many satisfactory results have been proven via Carleman estimates, and a thorough discussion can be found in the work of Eller, Isakov, Nakamura and Tataru, as well as that of Cheng, Isakov, Yamamoto and Zhou (see [7] and [5], respectively) and other authors. In the following two theorems, $u$ will be a solution of (1.1), $\Omega^{\prime}$ is an open domain in $\mathbb{R}^{3}$, and $T^{\prime}$ will be some positive real number (in practice, we will have $\Omega^{\prime}$ containing $\Omega$, and $T^{\prime}$ larger than $T(\Omega)$ ). Both of these results are from [7]; the former is a slightly simplified statement of Corollary 3.5, and the latter is Theorem 5.5. 
Theorem 3.2.2. (Eller, Isakov, Nakamura, Tataru)

Let $a_{1}=\frac{1}{\mu}, a_{2}=\frac{1}{\lambda+2 \mu}$ and assume, for some $\theta>0$, they both satisfy

$$
\theta^{2} a_{j}\left(a_{j}+a_{j}^{-1 / 2}\left|t \nabla a_{j}\right|\right)<a_{j}+1 / 2 x \cdot \nabla a_{j}
$$

and

$$
\theta^{2} a_{j} \leq 1
$$

on $\left[-T^{\prime}, T^{\prime}\right] \times \overline{\Omega^{\prime}}$, that $a_{j} \in C^{1}\left(\left[-T^{\prime}, T^{\prime}\right] \times \overline{\Omega^{\prime}}\right)$, and that $\Omega^{\prime} \subset B\left(0, \theta T^{\prime}\right)$. Then, if $u=0$ and $\partial_{\nu} u=0$ on $\left(-T^{\prime}, T^{\prime}\right) \times \partial \Omega^{\prime}$, then $u(t, x)=0$ when $|x|^{2}>\theta^{2} t^{2}$.

Theorem 3.2.3. Assume that the coefficients $\mu, \lambda \in C^{3}$ are time independent. Let $S$ be a noncharacteristic surface with respect to both $\square_{a_{1}}$ and $\square_{a_{2}}$. Then we have unique continuation across $S$ for $H_{l o c}^{1}$ solutions $u$ to (1.1).

With the assistance of these theorems, we conclude this section by proving a unique continuation result needed later for the reconstruction process. Essentially, we seek to answer the following question:

Suppose a solution $u$ of (1.1), with $u(0, x)=f(x)=0$ outside $\Omega$, also vanishes outside $\Omega$ at some later time $T$. From this, can we determine if $f(x)=0$ inside $\Omega$ as well? Because of finite propagation speed, this is certainly not the case for all times $T>0$ (let $T$ be small, and let the support of $f$ be, for instance, some small ball contained in $\Omega$ ), and so we seek to describe sufficient conditions for making such a determination.

Theorem 3.2.4 (Sufficient conditions for the Lamé Parameters). Suppose that $\mu, \lambda \in C^{3}$ are time independent, and that the maximum and minimum speeds of propagation satisfy the inequality

$$
c^{+}<3 c^{-}
$$

that there exist $\theta, T$, and $\epsilon>0$ so that

$$
\begin{aligned}
& \frac{1}{3} c^{+}<\theta<c^{-} \\
& T>\frac{2(R+\epsilon)}{3 \theta-c^{+}}
\end{aligned}
$$


and that $\left(a_{1}, a_{2}, T, \theta\right)$ satisfy the gradient condition (3.3) on

$$
\left[-\frac{3 T}{2}, \frac{3 T}{2}\right] \times \overline{B\left(0, R+\frac{T}{2} c^{+}+\epsilon\right)} .
$$

Assume also that the surface $S=\left\{(t, x):|x|^{2}=\theta^{2} t^{2}\right\}$ is non-characteristic for $\square_{a_{1}}, \square_{a_{2}}$. Then, for solutions $u$ of (1.1) (with $f$ compactly supported in $\Omega=B(0, R)$ ), if $u(T, x)=0$ for $x \notin \Omega$, we have $f(x)=0$.

Proof. By assumption, we know

$$
u(T, x)=0, \quad \text { for } x \notin \Omega,
$$

and since $f$ is compactly supported in $\Omega$, we know

$$
u(0, x)=0, \quad \text { for } x \notin \Omega
$$

as well. Thus, by finite speed of propagation, we then have both

$$
u(t, x)=0 \quad \text { when }|x|-R>c^{+}|T-t|
$$

and

$$
u(t, x)=0 \quad \text { when }|x|-R>c^{+}|t| .
$$

Combining these observations shows (see Figure 3.1)

$$
u(t, x)=0 \quad \text { when }|x|-R>\frac{T}{2} c^{+},-T / 2 \leq t \leq 3 T / 2 .
$$

Next, by time-reversal, $u$ extends to an even function of $t$, so, in fact, we have

$$
u(t, x)=0 \quad \text { when }|x|-R>\frac{T}{2} c^{+},-3 T / 2 \leq t \leq 3 T / 2 .
$$

Now, because $\theta<c^{-}$, we have $\theta^{2} a_{j} \leq 1$ for $j=1,2$, and by hypothesis, the gradient condition is satisfied on $\overline{B\left(0, R+\frac{T}{2} c^{+}+\epsilon\right)} \times\left[-\frac{3 T}{2}, \frac{3 T}{2}\right]$. Because $\theta>c^{+} / 3$ and $T>2(R+$ $\epsilon) /\left(3 \theta-c^{+}\right)$, we check that

$$
R+\frac{T}{2} c^{+}+\epsilon<\frac{T}{2}\left(3 \theta-c^{+}\right)+\frac{T}{2} c^{+}<\frac{3 T}{2} \theta
$$




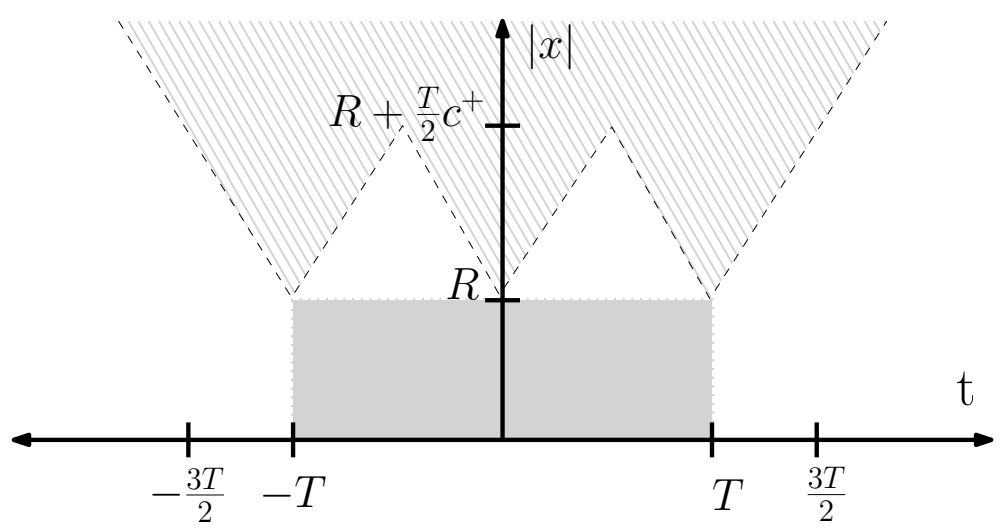

Figure 3.1: The solid gray region is $B(0, R) \times(-T, T)$; the dashed region where $u=0$ by finite speed of propagation and time-reversal.

so that we can apply Theorem 3.2.2, with $\Omega^{\prime}=B\left(0, R+\frac{T}{2} c^{+}+\epsilon\right)$ and $T^{\prime}=\frac{3 T}{2}$. Thus, $u(t, x)=0$ whenever $|x|^{2}>\theta^{2} t^{2}$, showing that $u(0, x)=0$, except possibly at the origin. Theorem 3.2.3 allows us to extend this solution uniquely, and thus $u(0,0)=0$ as well, showing $f \equiv 0$.

Before we continue, we make a few remarks about the conditions placed on the Lamé parameters in this theorem. First, the condition $c^{+}<3 c^{-}$is needed to guarantee we can choose $\theta$ so that $\frac{1}{3} c^{+}<\theta<c^{-}$; we need $\theta<c^{-}$to ensure condition (3.3) of Theorem 3.2.2 is met, and $\frac{1}{3} c^{+}<\theta$ is needed to ensure that the first inequality in (3.5) is valid. This condition essentially reads, "the speed of the S-wave must be more than a third that of the P-wave." For many materials, this is a realistic assumption (for instance, this is true of most earth materials), though as McLaughlin and Yoon note in [24], in biological tissue, this assumption may not be reasonable. In spite of this, because the observation surface is not a true discontinuity in the medium, it may be reasonable to expect that there is no exchange between the P- and S-modes, allowing us to work with a stronger form of unique continuation and drop the assumptions on the wave speeds. We will not pursue this issue any further in the present work, however.

The gradient condition is somewhat more subtle, though making some additional hy- 
potheses could help to simplify the situation. For instance, if we were able to assume that the medium is homogeneous outside some neighborhood of $\Omega$ (i.e., $\lambda$ and $\mu$ are constant, so that $\left.\nabla a_{j}=0\right)$, then the gradient condition simply reads $\theta<c^{-}$outside that neighborhood. Alternately, if we were to make assumptions on the size of $\left|\nabla a_{j}\right|$, say $\left|\nabla a_{j}\right|<\delta a_{j}$ for some $\delta>0$, then the condition could be reduced to

$$
\theta^{2}\left(a_{j}+a_{j}^{1 / 2} \delta|t|\right)+\frac{\delta}{2}|x|<1 .
$$

Again, at this point in time, we will leave such additional assumptions for future work, and continue with the hypotheses in the form stated in the theorem.

\subsection{Energy of Initial Data and Solutions}

As we have previously discussed, the success of the reconstruction process depends on good local energy decay, ensured by the non-trapping condition. Before we move on to the reconstruction process, we will find it useful to specifically define two kinds of energy spaces associated with (1.1); first for the initial data, and then for solutions. In what follows, $U$ is a domain in $\mathbb{R}^{3}$ (in practice, we will have $U=\Omega$ or $U=\mathbb{R}^{3}$ ). We will begin by defining an inner product for the first space.

For functions $f, g$, and a set $U$, define the $H_{D}$ inner product by

$$
(f, g)_{H_{D}(U)}:=\int_{U} \lambda(\nabla \cdot f)(\nabla \cdot g)+\mu \operatorname{tr}\left((\nabla f)(\nabla g)+(\nabla f)^{T}(\nabla g)\right) d x
$$

where tr indicates the trace. Specifically, we have

$$
\operatorname{tr}(\nabla f)(\nabla g)=\sum_{i, j=1}^{3} \frac{\partial f_{j}}{\partial x_{i}} \frac{\partial g_{i}}{\partial x_{j}} ; \quad \operatorname{tr}(\nabla f)^{T}(\nabla g)=\sum_{i, j=1}^{3} \frac{\partial f_{i}}{\partial x_{j}} \frac{\partial g_{i}}{\partial x_{j}} .
$$

It is easy to see that $(\cdot, \cdot)_{H_{D}(U)}$ is symmetric and bilinear. Furthermore,

Lemma 3.3.1. For functions $f, g \in C_{0}^{\infty}(U)$, we have

$$
(f, g)_{H_{D}(U)}=\langle P f, g\rangle_{L^{2}(U)}=\langle f, P g\rangle_{L^{2}(U)}
$$

Proof. Using the identities

$$
(\nabla \cdot(\mu(\nabla f))) \cdot g=\nabla \cdot((\mu(\nabla f)) g)-\mu \operatorname{tr}(\nabla f)(\nabla g)
$$




$$
\left(\nabla \cdot\left(\mu(\nabla f)^{T}\right)\right) \cdot g=\nabla \cdot\left(\left(\mu(\nabla f)^{T}\right) g\right)-\mu \operatorname{tr}(\nabla f)^{T}(\nabla g)
$$

and

$$
(\nabla(\lambda(\nabla \cdot f))) \cdot g=\nabla \cdot(\lambda(\nabla \cdot f) g)-\lambda(\nabla \cdot f)(\nabla \cdot g)
$$

we have

$$
\begin{aligned}
\langle P f, g\rangle_{L^{2}(U)}= & \int_{U} \lambda(\nabla \cdot f)(\nabla \cdot g)+\mu \operatorname{tr}\left((\nabla f)(\nabla g)+(\nabla f)^{T}(\nabla g)\right) d x \\
& -\int_{U} \nabla \cdot\left(\left(\mu\left((\nabla f)+(\nabla f)^{T}\right) g\right)\right)+\nabla \cdot(\lambda(\nabla \cdot f) g) d x
\end{aligned}
$$

Since $g \in C_{0}^{\infty}(U)$, the second integral is zero by the divergence theorem, and we have $\langle P f, g\rangle_{L^{2}(U)}=(f, g)_{H_{D}(U)}$. Similarly, using $f \in C_{0}^{\infty}(U)$ will show $(f, g)_{H_{D}(U)}=\langle f, P g\rangle_{L^{2}(U)}$.

Next, we define $H_{D}(U)$ to be the completion of $C_{0}^{\infty}(U)$ under the norm

$$
\|f\|_{H_{D}(U)}^{2}=(f, f)_{H_{D}(U)}
$$

(this quantity is essentially the total elastic energy of $f$ in $U$, with a factor of $1 / 2$ omitted for convenience), and then define the energy space

$$
\mathcal{H}(U)=H_{D}(U) \oplus L^{2}(U)
$$

with the norm

$$
\left\|\left(f_{1}, f_{2}\right)\right\|_{\mathcal{H}(U)}=\left\|f_{1}\right\|_{H_{D}(U)}+\left\|f_{2}\right\|_{L^{2}(U)} .
$$

Finally, we define the total energy for a function $u(t, x)$ at time $t$ as

$$
E_{U}(u, t)=\|u(t, \cdot)\|_{H_{D}(U)}^{2}+\left\|u_{t}(t, \cdot)\right\|_{L^{2}(U)}^{2},
$$

i.e., the total energy is the sum of the elastic and kinetic energies of $u$ at time $t$. Before moving on, we note that, as a consequence of these definitions, if $u$ solves (1.1) with $f \in$ $H_{D}(\Omega)$, then we have

$$
E_{\mathbb{R}^{3}}(u, 0)=\|f\|_{H_{D}\left(\mathbb{R}^{3}\right)}^{2}=\|f\|_{H_{D}(\Omega)}^{2}=E_{\Omega}(u, 0) .
$$




\subsection{Reconstruction}

Now that we have all the necessary preliminaries in place, we are in the position to investigate the reconstruction process. As suggested by our earlier discussion of techniques in TAT, one possible approach to reconstruction would be the use of time-reversal for solutions $v_{0}$ of

$$
\begin{cases}\left(\partial_{t}^{2}+P\right) v_{0} & =0 \quad \text { in }(0, T) \times \Omega, \\ \left.v_{0}\right|_{[0, T] \times \partial \Omega} & =h \\ \left.v_{0}\right|_{t=T} & =0 \\ \left.\partial_{t} v_{0}\right|_{t=T} & =0\end{cases}
$$

where $h=\Lambda f$, and then attempt to define an inverse $A_{0}$ by

$$
A_{0} h:=v_{0}(0, \cdot) \text { in } \bar{\Omega},
$$

with the hope that $A_{0} h=A_{0} \Lambda f$ approximates $f$. The problem with this method is that $h$ may not vanish on $\{t=T\} \times \partial \Omega$, causing the boundary conditions to be incompatible.

To correct this, we will need to introduce an error term, and modify our approach accordingly. Given $h$ (again, eventually $h=\Lambda f$ ), define $\phi$ and $v$ by

$$
\begin{gathered}
P \phi=0,\left.\quad \phi\right|_{\partial \Omega}=h(T, \cdot), \\
\begin{cases}\left(\partial_{t}^{2}+P\right) v=0 & \text { in }(0, T) \times \Omega, \\
\left.v\right|_{[0, T] \times \partial \Omega} & =h, \\
\left.v\right|_{t=T} & =\phi, \\
\left.\partial_{t} v\right|_{t=T} & =0,\end{cases}
\end{gathered}
$$

and note that the boundary data is now compatible to first order, in that $\phi=h$ on $\{t=T\} \times$ $\partial \Omega$. Now, we define the pseudo-inverse $A$ by

$$
A h:=v(0, \cdot) \quad \text { in } \bar{\Omega} .
$$


Our goal now is to show that $A$ maps the range of $\Lambda$ to $H_{D}(\Omega)$, and that the error $\|f-A \Lambda f\|_{H_{D}(\Omega)}$ is small compared to $\|f\|_{H_{D}(\Omega)}$; in particular, we will show that the operator $K=(I-A \Lambda)$ is a contraction on $H_{D}(\Omega)$. The following theorem is our main result. Recall that we say $(\Omega, P)$ is non-trapping if every ray starting in $\Omega$ exits in finite time, and the supremum of these times (which we denote by $T(\Omega)$ ) is finite as well (see page 19 for more discussion).

Theorem 3.4.1. Suppose that $f$ is compactly supported in $\Omega$, that $(\Omega, P)$ is non-trapping and satisfies the hypotheses of Theorem 3.2.4, with $T>T(\Omega)$. Then $A \Lambda=I-K$, where $K$ is compact in $H_{D}(\Omega)$ and $\|K\|_{H_{D}(\Omega)}<1$. In particular, $I-K$ is invertible on $H_{D}(\Omega)$, and we have the following Neumann expansion for $f$ :

$$
f=\sum_{m=0}^{\infty} K^{m} A h, \quad h:=\Lambda f .
$$

Proof. Let $w$ solve

$$
\begin{cases}\left(\partial_{t}^{2}+P\right) w & =0 \quad \text { in }(0, T) \times \Omega \\ \left.w\right|_{[0, T] \times \partial \Omega} & =0 \\ \left.w\right|_{t=T} & =\left.u\right|_{t=T}-\phi \\ \left.\partial_{t} w\right|_{t=T} & =\left.\partial_{t} u\right|_{t=T} .\end{cases}
$$

Let $v$ be the solution of (3.7) with $h=\Lambda f$. Then $v+w$ will solve same initial boundary value problem as $u$ (with initial conditions given at $t=T$ ), and thus $u=v+w$. For $t=0$, we then have

$$
f=A \Lambda f+w(0, \cdot)
$$

and so,

$$
K f=w(0, \cdot) .
$$

For convenience of notation, let $u^{T}=u(T, \cdot)$ and $u_{t}^{T}=u_{t}(T, \cdot)$. Because $u^{T}=\phi$ on $\partial \Omega$, and because $P \phi=0$,

$$
\left(u^{T}-\phi, \phi\right)_{H_{D}(\Omega)}=\left\langle u^{T}-\phi, P \phi\right\rangle_{L^{2}(\Omega)}=0 .
$$


Thus,

$$
\left\|u^{T}-\phi\right\|_{H_{D}(\Omega)}^{2}=\left\|u^{T}\right\|_{H_{D}(\Omega)}^{2}-\|\phi\|_{H_{D}(\Omega)}^{2} \leq\left\|u^{T}\right\|_{H_{D}(\Omega)}^{2}
$$

showing that

$$
E_{\Omega}(w, T)=\left\|u^{T}-\phi\right\|_{H_{D}(\Omega)}^{2}+\left\|u_{t}^{T}\right\|_{L^{2}(\Omega)}^{2} \leq\left\|u^{T}\right\|_{H_{D}(\Omega)}^{2}+\left\|u_{t}^{T}\right\|_{L^{2}(\Omega)}^{2}=E_{\Omega}(u, T) .
$$

Because $w=0$ on $\partial \Omega$, we have $(w, w)_{H_{D}(\Omega)}=\langle P w, w\rangle_{L^{2}(\Omega)}$ so that

$$
\begin{aligned}
\frac{d}{d t} E_{\Omega}(w, t) & =\frac{d}{d t}\langle P w, w\rangle_{L^{2}(\Omega)}+\frac{d}{d t}\left\langle w^{\prime}, w^{\prime}\right\rangle_{L^{2}(\Omega)} \\
& =\left\langle P w^{\prime}, w\right\rangle_{L^{2}(\Omega)}+\left\langle P w, w^{\prime}\right\rangle_{L^{2}(\Omega)}+\left\langle w^{\prime \prime}, w^{\prime}\right\rangle_{L^{2}(\Omega)}+\left\langle w^{\prime}, w^{\prime \prime}\right\rangle_{L^{2}(\Omega)} \\
& =\left\langle P w^{\prime}, w\right\rangle_{L^{2}(\Omega)}+\left\langle w^{\prime}, w^{\prime \prime}\right\rangle_{L^{2}(\Omega)} \\
& =\left\langle w^{\prime}, P w\right\rangle_{L^{2}(\Omega)}+\left\langle w^{\prime}, w^{\prime \prime}\right\rangle_{L^{2}(\Omega)}=0
\end{aligned}
$$

(note that this is just a reflection of the fact that the Dirichlet boundary conditions imposed on $w$ are energy-conserving). Therefore,

$$
E_{\Omega}(w, 0)=E_{\Omega}(w, T) \leq E_{\Omega}(u, T) \leq E_{\mathbb{R}^{3}}(u, T)=E_{\Omega}(u, 0)=\|f\|_{H_{D}(\Omega)}^{2},
$$

showing

$$
\|K f\|_{H_{D}(\Omega)}^{2}=\|w(0, \cdot)\|_{H_{D}(\Omega)}^{2} \leq E_{\Omega}(w, 0) \leq\|f\|_{H_{D}(\Omega)}^{2} .
$$

We next seek to show that this inequality is, in fact, strict. So, suppose that there is some $f$ so that $\|K f\|_{H_{D}(\Omega)}=\|f\|_{H_{D}(\Omega)}$. This implies that all the above inequalities are actually equalities as well, and in particular, $E_{\Omega}(u, T)=E_{\mathbb{R}^{3}}(u, T)$, so that

$$
u(T, x)=0, \quad \text { for } x \notin \Omega .
$$

By Theorem 3.2.4, we then have $f=0$, and thus $\|K f\|_{H_{D}(\Omega)}<\|f\|_{H_{D}(\Omega)}$ for all non-zero $f \in H_{D}(\Omega)$.

Now, we will show that $K$ is compact. We have that $u(T, \cdot)$ and $u_{t}(T, \cdot)$ are smooth as functions on $\bar{\Omega}$ because $T>T(\Omega)$ (all singularities beginning in $\Omega$ will have left), and so, 
as linear operators acting on $f$, they have smooth Schwartz kernels. This will also imply $\phi$ is smooth, by elliptic regularity. From this, we can see that the map

$$
H_{D}(\Omega) \rightarrow \mathcal{H}(U): f \mapsto\left(u^{T}-\phi, u_{t}^{T}\right)
$$

has a smooth kernel, and is therefore compact (see Proposition 2.2.3). Next, we note that the solution operator of (3.7) from $t=T$ to $t=0$ (i.e., the map $\left(u^{T}-\phi, u_{t}^{T}\right) \mapsto\left(w(0, \cdot), w_{t}(0, \cdot)\right)$ is bounded in $\mathcal{H}(U)$ (unitary actually, because $E_{\Omega}(w, T)=E_{\Omega}(w, 0)$ ). Thus, we conclude that the map $K: H_{D}(\Omega) \rightarrow H_{D}(\Omega), f \mapsto w(0, \cdot)$ is compact, as the composition of a compact and a bounded map, finally allowing us to conclude $\|K\|_{H_{D}(\Omega)}<1$ by Proposition 2.2.7, and the proof is complete.

It is additionally worth noting that, in addition to uniqueness, the proof of this theorem also gives insight into the stability of this problem. In particular, we have

$$
\|K f\|_{H_{D}(\Omega)} \leq\left(\frac{E_{\Omega}(u, T)}{E_{\Omega}(u, 0)}\right)^{1 / 2}\|f\|_{H_{D}(\Omega)}, \quad \forall f \in H_{D}(\Omega), f \neq 0,
$$

providing a bound on $\|K\|_{H_{D}(\Omega)}$ in terms of the local energy decay. Furthermore, because $f-A \Lambda f=K f$, this also describes the error in the reconstruction if we only use the first term $K^{0} A h=A \Lambda f$ of the Neumann series. Additionally, if $T$ is chosen so that the hypotheses of Theorem 3.2.4 are satisfied, but $T \leq T(\Omega)$, then we can still conclude that $\|K f\|_{H_{D}(\Omega)}<\|f\|_{H_{D}(\Omega)}$ for all non-zero $f$, but we can no longer be sure $K$ is compact, and thus have no reason to expect $\|K\|_{H_{D}(\Omega)}$ is strictly less than 1 . Finally, if $T<\frac{R}{c^{+}}$, then one can find a non-zero $f$ so that $\|K f\|_{H_{D}(\Omega)}=\|f\|_{H_{D}(\Omega)}$ (by choosing an $f$ supported in a small enough ball, finite speed of propagation will imply $u(T, x)=0$ outside of $\Omega$ ). Actually, we can refine this a bit: if $\operatorname{dist}(x, \partial \Omega)$ is the length of the shortest geodesic from $x$ to the boundary, we set

$$
T_{0}:=\max _{x \in \bar{\Omega}} \operatorname{dist}(x, \partial \Omega) .
$$

Then, if $T<T_{0}$, the set $\{x \in \Omega: \operatorname{dist}(x, \partial \Omega)>T\}$ is non-empty, and for any $f$ supported in that set, we will have $\Lambda f=0$. Thus, uniqueness fails for $T<T_{0}$. Unlike the scalar case however, $T \geq T_{0}$ is not enough to ensure uniqueness; one could take non-zero initial data 
with zero P-mode, supported in a set such that the distance from that set to the boundary at the $\mathrm{S}$-wave speed is greater than $T_{0}$, and have $\Lambda f=0$. Thus it will also be important to consider an analogous time $T_{1}$, related to the slower $\mathrm{S}$-wave speed. This idea will be further discussed and developed in Chapter 5 when we conduct our numerical experimentation. 
Chapter 4

\section{BACKGROUND ON NUMERICAL WORK}

In the previous chapter it was shown that, if the Lamé parameters satisfy a condition on their relative size (briefly, that $\sup \sqrt{\mu}<3 \inf \sqrt{\lambda+2 \mu}$ ) and one regarding their gradients, then $f$ is recoverable via a Neumann series. Our goal in this and the following chapter is to numerically implement the time-reversal method just outlined. We devote this chapter to discussing the numerical methods involved, and in the following chapter, we present the results of our numerical experimentation.

\subsection{Finite Difference Methods}

When computing solutions to partial differential equations, it is, of course, usually not possible to write closed-form, exact answers. To overcome this, many techniques have been developed that produce good approximations to solutions, such as finite difference methods, finite element methods, and finite volume methods. In our work, we will implement finite difference methods to construct approximate solutions; we describe some of their basic workings and features in this section.

\subsubsection{Finite differences}

Suppose we want to approximate $f^{\prime}$. From the Taylor series, we have

$$
f\left(t_{0}+k\right)=f\left(t_{0}\right)+k f^{\prime}\left(t_{0}\right)+O\left(k^{2}\right)
$$

and so, for small $k$, we can approximate $f^{\prime}$ by

$$
f^{\prime}(t)=\frac{f(t+k)-f(t)}{k}+O(k) .
$$

This is called the forward difference approximation for $f^{\prime}$. We can also approximate $f^{\prime}$ using either the backward difference approximation

$$
f^{\prime}(t)=\frac{f(t)-f(t-k)}{k}+O(k)
$$


or the central difference approximation

$$
f^{\prime}(t)=\frac{f\left(t+\frac{1}{2} k\right)-f\left(t-\frac{1}{2} k\right)}{k}+O\left(k^{2}\right) .
$$

To see why the central difference gives us better accuracy, write

$$
f\left(t_{0}+\frac{1}{2} k\right)=f\left(t_{0}\right)+\frac{1}{2} k f^{\prime}\left(t_{0}\right)+\frac{1}{8} k^{2} f^{\prime \prime}\left(t_{0}\right)+O\left(k^{3}\right)
$$

and

$$
f\left(t_{0}-\frac{1}{2} k\right)=f\left(t_{0}\right)-\frac{1}{2} k f^{\prime}\left(t_{0}\right)+\frac{1}{8} k^{2} f^{\prime \prime}\left(t_{0}\right)+O\left(k^{3}\right)
$$

and note that $f\left(t_{0}+\frac{1}{2} k\right)-f\left(t_{0}-\frac{1}{2} k\right)=k f^{\prime}\left(t_{0}\right)+O\left(k^{3}\right)$.

If we want to be more accurate, then we can take additional terms of the Taylor series. For example, if we want to improve the accuracy of our forward finite difference approximation to $O\left(k^{2}\right)$, we consider the Taylor expansions

$$
\begin{gathered}
f\left(t_{0}+k\right)=f\left(t_{0}\right)+k f^{\prime}\left(t_{0}\right)+\frac{1}{2} k^{2} f^{\prime \prime}\left(t_{0}\right)+O\left(k^{3}\right), \\
f\left(t_{0}+2 k\right)=f\left(t_{0}\right)+2 k f^{\prime}\left(t_{0}\right)+2 k^{2} f^{\prime \prime}\left(t_{0}\right)+O\left(k^{3}\right) .
\end{gathered}
$$

Then, we take a combination of these such that the $f^{\prime \prime}$ terms cancel:

$$
2 f\left(t_{0}+k\right)-\frac{1}{2} f\left(t_{0}+2 k\right)=\frac{3}{2} f\left(t_{0}\right)+k f^{\prime}\left(t_{0}\right)+O\left(k^{3}\right),
$$

so that

$$
f^{\prime}(t)=\frac{-\frac{3}{2} f(t)+2 f(t+k)-\frac{1}{2} f(t+2 k)}{k}+O\left(k^{2}\right),
$$

and we have the second-order accurate forward difference approximation of $f^{\prime}$.

If we wish to approximate higher-order derivatives, we can use finite differences of lowerorder derivatives. For example, if we wish to find a second-order accurate central difference approximation of $f^{\prime \prime}$, we compute

$$
\begin{aligned}
f^{\prime \prime}(t) & =\frac{f^{\prime}\left(t+\frac{1}{2} k\right)-f^{\prime}\left(t-\frac{1}{2} k\right)}{k}+O\left(k^{2}\right) \\
& =\frac{\frac{f(t+k)-f(t)}{k}-\frac{f(t)-f(t-k)}{k}}{k}+O\left(k^{2}\right) \\
& =\frac{f(t+k)-2 f(t)+f(t-k)}{k^{2}}+O\left(k^{2}\right) .
\end{aligned}
$$

By adapting these two methods appropriately, we can construct forward, backward, or central difference approximations of any order, and any accuracy. 


\subsubsection{Finite differences on a grid}

Given a fixed $h$, we define a grid of spacing $h$ to be the set of points $\left\{x_{l}\right\}_{l=-\infty}^{\infty}$ given by $x_{l}=l h$. We may also wish to definite variations of this, such as finite grids $\left\{x_{l}\right\}_{l=0}^{L}$, $x_{l}=x_{0}+l h\left(\right.$ for a fixed $\left.x_{0}, L\right)$ or semi-infinite grids (of spacing $k$ ) $\left\{t_{n}\right\}_{n=0}^{\infty}, t_{n}=n k$, and will make the distinction clear when necessary. We will also wish to consider products of grids, and will refer to $\left\{t_{n}\right\}_{n=0}^{\infty} \times\left\{x_{l}\right\}_{l=-\infty}^{\infty}$, for instance, as a grid of spacing $k, h$. Finally, if $u$ is a function on a grid, we will abbreviate $u\left(t_{n}, x_{l}\right)$ as $u_{l}^{n}$, and $u\left(t_{n}, \cdot\right)$ as $u^{n}$.

Finite difference schemes and grids offer a natural (and easily programmable) means of approximating solutions to partial differential equations. To illustrate this by way of example, we seek to construct approximate solutions of the heat equation

$$
u_{t}=u_{x x}
$$

on $\{t \geq 0\} \times\{0 \leq x \leq 1\}$ with initial condition $u(0, x)=f(x)$ and boundary conditions $u(t, 0)=u(t, 1)=0$. We fix a grid of spacing $k, h\left\{t_{n}\right\}_{n=0}^{\infty} \times\left\{x_{l}\right\}_{l=0}^{L}$ (here, we assume $h$ and $L$ are chosen so that $\left.x_{L}=1\right)$.

If we approximate $u_{t}$ using a first-order accurate forward difference method, and $u_{x x}$ using a second-order accurate centered difference method, we have

$$
\frac{u_{l}^{n+1}-u_{l}^{n}}{k}=\frac{u_{l+1}^{n}-2 u_{l}^{n}+u_{l-1}^{n}}{h^{2}}
$$

so that

$$
u_{l}^{n+1}=u_{l}^{n}+\frac{k}{h^{2}}\left(u_{l+1}^{n}-2 u_{l}^{n}+u_{l-1}^{n}\right) \quad: 0<l<L,
$$

and we can implement our boundary condition as

$$
u_{l}^{n+1}=0: l=0, L \text {. }
$$

Thus, we have an explicit formula for $u_{l}^{n+1}$ in terms of the values of $u$ at the previous time step. To implement the initial condition, we sample $f$ on our grid to determine $u^{0}$, and now we can use this scheme to compute $u_{l}^{n}$ for any $n, l$. This is known as a one-step scheme: computing $u^{n+1}$ only requires the values of $u^{n}$, and thus for one-step schemes, knowing $u^{0}$ allows one to calculate $u^{n}$ for all $n$. We will discuss two-step methods in a later section. 
Whether or not this scheme will yield accurate or useful approximations to actual solutions, however, is a different question. To quantify this, we will first need to define what it means to say a scheme is convergent.

\subsubsection{Convergence, consistency and stability}

Let us begin by making precise the idea of convergence for one-step finite difference schemes. We say such a scheme approximating a partial differential equation is convergent if, for every solution $u(t, x)$ to the partial differential equation, with initial data $u_{0}(x)$, if $v$ is a

solution of the finite difference scheme such that $v_{l}^{0}$ converges to $u_{0}(x)$ as $x_{l} \rightarrow x$, then $v_{l}^{n}$ converges to $u(t, x)$ as $\left(t_{n}, x_{l}\right) \rightarrow(t, x)$ as $h, k \rightarrow 0$.

Unfortunately, it can be difficult to prove convergence of a scheme directly, and so alternative methods must be found. There are two related notions, consistency and stability, that are not only naturally desirable properties for a scheme to have, but are also easier to work with, and as we will eventually see, can be used to assess convergence of a scheme.

We begin by defining the local truncation error as the difference of the original differential equation and the finite difference approximation when applied to a smooth function $\phi$. For instance, the local truncation error of the first-order accurate forward difference approximation of $f^{\prime}$ is

$$
\begin{aligned}
\phi^{\prime}(t) & -\frac{\phi(t+k)-\phi(t)}{k} \\
& =\frac{1}{2} k \phi^{\prime \prime}(t)+O\left(k^{2}\right) \\
& =O(k) .
\end{aligned}
$$

In the case of our scheme for the heat equation, the truncation error is

$$
\begin{aligned}
\phi_{t}(t, x) & -\phi_{x x}(t, x)-\frac{\phi(t+k, x)-\phi(t, x)}{k}+\frac{\phi(t, x+h)-2 \phi(t, x)+\phi(t, x-h)}{h^{2}} \\
& =\phi_{t}(t, x)-\phi_{x x}(t, x)-\phi_{t}(t, x)+O(k)+\phi_{x x}(t, x)+O\left(h^{2}\right) \\
& =O(k)+O\left(h^{2}\right),
\end{aligned}
$$

and so overall is $O\left(k, h^{2}\right)$. Alternatively, if $\phi=u$ is an exact solution of the differential equation, we can view the local truncation error as the error in applying the finite difference scheme to $u$. 
For a particular finite difference scheme, if the local truncation error goes to zero as $k, h \rightarrow 0$, we say the scheme is consistent. In some sense, consistency ensures that exact solutions to the differential equation will also be solutions of the finite difference scheme. For both of the examples just discussed, it is clear that the schemes are consistent.

Next, we want to get a sense of how error resulting from an early step in the finite difference scheme will manifest later; ideally, we would like this later error to be bounded. To analyze this, we introduce the notion of stability, following the presentation given in [34].

Given a finite difference scheme for a first-order (in $t$ ) equation and a fixed $k, h$, we say the scheme is stable for $k, h$ if there is an integer $J$ such that, for any positive $N$, there exists a constant $C_{N}$ such that

$$
h \sum_{l=-\infty}^{\infty}\left|u_{l}^{n}\right|^{2} \leq C_{N} h \sum_{j=0}^{J} \sum_{l=-\infty}^{\infty}\left|u_{l}^{j}\right|^{2}
$$

for all $n \leq N$. We can also adapt this definition in an obvious way for finite grids.

For many schemes, we will be able to take $J=0$ in the above definition so that the stability condition reads

$$
h \sum_{l=-\infty}^{\infty}\left|u_{l}^{n}\right|^{2} \leq C_{N} h \sum_{l=-\infty}^{\infty}\left|u_{l}^{0}\right|^{2}
$$

or

$$
h \sum_{l=0}^{L}\left|u_{l}^{n}\right|^{2} \leq C_{N} h \sum_{l=0}^{L}\left|u_{l}^{0}\right|^{2}
$$

for finite grids.

If we think temporarily of $u^{n}$ as a function that is piecewise constant on the intervals $\left[x_{l}, x_{l+1}\right)$, then its $L^{2}$ norm is

$$
\begin{aligned}
\left(\int\left|u^{n}(x)\right|^{2} d x\right)^{1 / 2} & =\left(\sum_{l=-\infty}^{\infty} \int_{x_{l}}^{x_{l+1}}\left|u_{l}^{n}\right|^{2} d x\right)^{1 / 2} \\
& =\left(\sum_{l=-\infty}^{\infty} h\left|u_{l}^{n}\right|^{2}\right)^{1 / 2} \\
& =\left(h \sum_{l=-\infty}^{\infty}\left|u_{l}^{n}\right|^{2}\right)^{1 / 2}
\end{aligned}
$$


With this in mind, for a function $v$ defined on a grid $\left\{x_{l}\right\}$ we denote

$$
\|v\|_{h}=\left(h \sum_{l}\left|v_{l}\right|^{2} d x\right)^{1 / 2}
$$

and now the stability condition (with $J=0$, and $C_{N}$ replacing $\sqrt{C_{N}}$ ) reads

$$
\left\|u^{n}\right\|_{h} \leq C_{N}\left\|u^{0}\right\|_{h}
$$

We conclude this subsection by stating the following theorem, which describes precisely the relation between consistency, stability, and convergence.

Theorem 4.1.1 (Lax equivalence theorem). For a consistent finite difference method for a well-posed, linear initial value problem, the scheme is convergent if and only if it is stable.

Thus, consistency and stability are exactly the conditions we seek in selecting a useful finite difference scheme: if a consistent scheme is stable, then it is convergent, and further, there are no non-stable schemes worth considering, as they will necessarily fail to be convergent.

\subsubsection{Von Neumann analysis}

Now that we have established a relationship between stable schemes and convergent ones, we present a powerful method of testing the stability of a particular finite difference scheme, known as von Neumann analysis. We illustrate this with an example, the one-way, 1dimensional wave equation

$$
u_{t}=c u_{x}
$$

on $\mathbb{R}^{+} \times \mathbb{R}$ where $c$ is the (constant) wave speed. We develop a forward-time, forward-space finite difference scheme

$$
\begin{aligned}
u_{l}^{n+1} & =u_{l}^{n}+c \frac{k}{h}\left(u_{l+1}^{n}-u_{l}^{n}\right) \\
& =(1-\lambda) u_{l}^{n}+\lambda u_{l+1}^{n}
\end{aligned}
$$

where $\lambda=c \frac{k}{h}$. 
Before we proceed, it will be necessary to recall some facts and defnitions pertaining to the Fourier transform. If $v$ is a function defined on the integers (i.e. a grid of spacing 1), then its Fourier transform (sometimes called its discrete-time Fourier transform) is given by

$$
\widehat{v}(\xi)=\frac{1}{\sqrt{2 \pi}} \sum_{l=-\infty}^{\infty} e^{-i l \xi} v_{l},
$$

and an inversion formula is given by

$$
v_{l}=\frac{1}{\sqrt{2 \pi}} \int_{-\pi}^{\pi} e^{i l \xi} \widehat{v}(\xi) d \xi
$$

If we switch our attention to infinite grids of spacing $h$, we can redefine the transform and inverse transform by

$$
\begin{aligned}
\widehat{v}(\xi) & =\frac{1}{\sqrt{2 \pi}} \sum_{l=-\infty}^{\infty} h e^{-i l h \xi} v_{l}, \\
v_{l} & =\frac{1}{\sqrt{2 \pi}} \int_{-\pi / h}^{\pi / h} e^{i l h \xi} \widehat{v}(\xi) d \xi
\end{aligned}
$$

We note that, for finite grids, we have the discrete Fourier transform at our disposal, though we omit further discussion.

Next, we recall Parseval's identity, which states that the $L^{2}$ norm of a function is equal to the $L^{2}$ norm of its Fourier transform. Specifically,

$$
\|v\|_{h}^{2}=h \sum_{l}\left|v_{l}\right|^{2}=\int_{-\pi / h}^{\pi / h}|\widehat{v}(\xi)|^{2} d \xi=\|\widehat{v}\|_{h}^{2} .
$$

Now, returning to our finite difference scheme, for each $n$, we can compute $\widehat{u^{n}}$, and we further observe

$$
\begin{aligned}
u_{l+1}^{n} & =\frac{1}{\sqrt{2 \pi}} \int_{-\pi / h}^{\pi / h} e^{i(l+1) h \xi \widehat{u^{n}}}(\xi) d \xi \\
& =\frac{1}{\sqrt{2 \pi}} \int_{-\pi / h}^{\pi / h} e^{i l h \xi} e^{i h \xi \widehat{u^{n}}}(\xi) d \xi
\end{aligned}
$$

and similarly for $u_{l-1}^{n}$. 
Now, if we rewrite our scheme in terms of the Fourier transforms of $u_{l}^{n}$ and $u_{l+1}^{n}$, we have

$$
u_{l}^{n+1}=\frac{1}{\sqrt{2 \pi}} \int_{-\pi / h}^{\pi / h} e^{i l h \xi}\left((1-\lambda)+\lambda e^{i h \xi}\right) \widehat{u^{n}}(\xi) d \xi .
$$

We also know that

$$
u_{l}^{n+1}=\frac{1}{\sqrt{2 \pi}} \int_{-\pi / h}^{\pi / h} e^{i l h \xi} \widehat{u^{n+1}}(\xi) d \xi,
$$

and so by uniqueness of Fourier transforms, we know

$$
\widehat{u^{n+1}}(\xi)=\left((1-\lambda)+\lambda e^{i h \xi}\right) \widehat{u^{n}}(\xi) .
$$

We call $g=(1-\lambda)+\lambda e^{i h \xi}$ the amplification factor, as advancing from one time-step to the next will amplify the Fourier transform of the solution by $g$. In particular, given initial data $u_{l}^{0}$, we will have

$$
\widehat{u^{n}}(\xi)=g^{n} \widehat{u^{0}}(\xi)
$$

so that

$$
\left\|u^{n}\right\|_{h}^{2}=\left\|\widehat{u^{n}}\right\|_{h}^{2}=\left\|g^{n} \widehat{u^{0}}\right\|_{h}^{2}=\int_{-\pi / h}^{\pi / h}|g|^{2 n}\left|\widehat{u^{0}}(\xi)\right|^{2} d \xi .
$$

Thus, in determining stability of a system, it will be useful to estimate the size of $|g|$. In particular, if $|g| \leq 1$, then

$$
\left\|u^{n}\right\|_{h}^{2} \leq \int_{-\pi / h}^{\pi / h}\left|\widehat{u^{0}}(\xi)\right|^{2} d \xi=\left\|\widehat{u^{0}}\right\|_{h}^{2}=\left\|u^{0}\right\|_{h}^{2}
$$

and the scheme will be stable.

So, we compute

$$
\begin{aligned}
|g|^{2} & =\left((1-\lambda)+\lambda e^{i h \xi}\right)\left((1-\lambda)+\lambda e^{-i h \xi}\right) \\
& =(1-\lambda)^{2}+\lambda^{2}+\lambda(1-\lambda)\left(e^{i h \xi}+e^{-i h \xi}\right) \\
& =(1-\lambda)^{2}+\lambda^{2}+2 \lambda(1-\lambda) \cos (h \xi) \\
& =1-2 \lambda(1-\lambda)+2 \lambda(1-\lambda) \cos (h \xi) \\
& =1-\lambda(1-\lambda) \sin ^{2}\left(\frac{1}{2} h \xi\right) .
\end{aligned}
$$


If we want $|g| \leq 1$ for all $\xi$, we then need $0 \leq \lambda \leq 1$. Therefore, we must have $c>0$, and must have $k<\frac{1}{c} h$ for the scheme to be stable.

\subsubsection{Two-step methods and stability}

Suppose now, that we wish to solve a typical wave equation

$$
u_{t t}=c^{2} u_{x x}
$$

with initial data $u(0, x)=u_{0}(x), u_{t}(0, x)=0$ on $\mathbb{R}^{+} \times \mathbb{R}$.

We can use the centered-space and centered-time finite differences to get the second-order accurate scheme

$$
u_{l}^{n+1}=2 u_{l}^{n}-u_{l}^{n-1}+c^{2} \frac{k^{2}}{h^{2}}\left(u_{l+1}^{n}-2 u_{l}^{n}+u_{l-1}^{n}\right) .
$$

Because $u^{n+1}$ depends explicitly on the values of $u^{n}$ and $u^{n-1}$, we call this a two-step method. In this case, simply knowing $u^{0}$ will not be enough to calculate all future values of $u^{n}$; we will have to either specify or otherwise approximate $u^{1}$ in order to proceed.

One way of doing this is via the Taylor series. At time $t=k($ i.e. $n=1$ ), we have

$$
\begin{aligned}
u(k, x) & =u(0, x)+k u_{t}(0, x)+\frac{1}{2} k^{2} u_{t t}(0, x)+O\left(k^{3}\right) \\
& =u_{0}(x)+\frac{1}{2} k^{2} c^{2} u_{x x}(0, x)
\end{aligned}
$$

because $u_{t}(0, x)=0$, and because $u_{t t}=c^{2} u_{x x}$. Then, if we use a centered finite difference for $u_{x x}$, we have

$$
u^{1}=u^{0}+c^{2} \frac{1}{2} \frac{k^{2}}{h^{2}}\left(u_{l+1}^{0}-2 u_{l}^{0}+u_{l-1}^{0}\right),
$$

and we can compute subsequent values of $u^{n}$ using our scheme.

We modify our definition of stability slightly to allow linear growth in $t$ for second-order equations; we say a two-step scheme is stable if there is an integer $J$ such that for any positive $N$, there exists a constant $C_{N}$ such that

$$
\left\|u^{n}\right\|_{h}^{2} \leq\left(1+n^{2}\right) C_{N} \sum_{j=0}^{J}\left\|u^{j}\right\|_{h}^{2}
$$


for all $n \leq N$.

In this case, we will always need $J$ at least 1 , because we have to specify (or estimate) our data at two time steps for these types of equations.

We consider the von Neumann analysis of our scheme; as before, there will be some amplification factor $g$ so that $\widehat{u^{n+1}}=g \widehat{u^{n}}$, and additionally, that $\widehat{u^{n-1}}=g^{-1} \widehat{u^{n}}$. Thus, in taking the Fourier transform of our scheme, we will have

$$
\left(g-2+g^{-1}\right) \widehat{u^{n}}=\lambda^{2}\left(e^{i h \xi}-2+e^{-i h \xi}\right) \widehat{u^{n}}
$$

where $\lambda^{2}=c^{2} \frac{k^{2}}{h^{2}}$. Then, using basic trigonometric identities,

$$
g-2+g^{-1}=-4 \lambda^{2} \sin ^{2}\left(\frac{1}{2} h \xi\right)
$$

so that

$$
\begin{aligned}
\left(g^{1 / 2}-g^{-1 / 2}\right)^{2} & =-4 \lambda^{2} \sin ^{2}\left(\frac{1}{2} h \xi\right) \\
g^{1 / 2}-g^{-1 / 2} & = \pm i 2 \lambda \sin \left(\frac{1}{2} h \xi\right) \\
g \pm i 2 \lambda \sin \left(\frac{1}{2} h \xi\right) g^{1 / 2}-1 & =0
\end{aligned}
$$

which is a quadratic for $g^{1 / 2}$, leading to solutions

$$
g=\left(\sqrt{1-\lambda^{2} \sin ^{2}\left(\frac{1}{2} h \xi\right)} \pm i \lambda \sin \left(\frac{1}{2} h \xi\right)\right)^{2} .
$$

From this, we can see that if $\lambda \leq 1$ (i.e. if $c k \leq h$ ), then $|g| \leq 1$, and this scheme will be stable.

These schemes extend to higher dimensions in a straightforward manner. If we wish to solve, for instance

$$
u_{t t}=c^{2}\left(u_{x x}+u_{y y}\right)
$$

writing $u\left(t_{n}, x_{l}, y_{m}\right)=u_{l, m}^{n}$, we can write the scheme

$$
u_{l, m}^{n+1}=2 u_{l, m}^{n}-u_{l, m}^{n-1}+c^{2} \frac{k^{2}}{h^{2}}\left(u_{l+1, m}^{n}-2 u_{l, m}^{n}+u_{l-1, m}^{n}+u_{l, m+1}^{n}-2 u_{l, m}^{n}+u_{l, m-1}^{n}\right)
$$


(there are, of course, other schemes one could choose). If one fixes a spatial grid of spacing $h$ (in both $x$ and $y$ ), the stability condition for this system is $c k \leq \frac{1}{\sqrt{2}} h$.

The stability analysis for systems of partial differential equations and for variable coefficients is more complicated, and we will state results for the specific schemes we wish to use in the next section, rather than discussing them in general.

\subsection{Our Methods}

Now we introduce the specific finite difference schemes we will use to approximate solutions to $1.1,3.7$ and 3.6 .

We wish to develop a scheme for the two-dimensional isotropic elastic wave equation

$$
\begin{gathered}
\left(u_{t t}\right)_{1}=\partial_{1}\left((\lambda+2 \mu) \partial_{1} u_{1}\right)+\partial_{2}\left(\mu \partial_{2} u_{1}\right)+\partial_{1}\left(\lambda \partial_{2} u_{2}\right)+\partial_{2}\left(\mu \partial_{1} u_{2}\right) \\
\left(u_{t t}\right)_{2}=\partial_{2}\left(\lambda \partial_{1} u_{1}\right)+\partial_{1}\left(\mu \partial_{2} u_{1}\right)+\partial_{2}\left((\lambda+2 \mu) \partial_{2} u_{2}\right)+\partial_{1}\left(\mu \partial_{1} u_{2}\right) .
\end{gathered}
$$

To simplify the notation somewhat, we write $u=u_{1}, v=u_{2}$ so that the system of partial differential equations becomes

$$
\begin{gathered}
u_{t t}=\partial_{1}\left((\lambda+2 \mu) \partial_{1} u\right)+\partial_{2}\left(\mu \partial_{2} u\right)+\partial_{1}\left(\lambda \partial_{2} v\right)+\partial_{2}\left(\mu \partial_{1} v\right) \\
v_{t t}=\partial_{2}\left(\lambda \partial_{1} u\right)+\partial_{1}\left(\mu \partial_{2} u\right)+\partial_{2}\left((\lambda+2 \mu) \partial_{2} v\right)+\partial_{1}\left(\mu \partial_{1} v\right),
\end{gathered}
$$

and use the convention $u\left(t_{n}, x_{l}, y_{m}\right)=u_{l, m}^{n}$ and similarly for $v$. To develop a scheme, we follow the method propopsed by Kelly et al. in [22] and further explored by Nilsson et al. in [25], and approximate spatial derivatives in the following way: for those terms where both derivatives are with respect to the same variable, we use

$$
\partial_{1}\left(\alpha \partial_{1} u\right) \simeq D_{1}^{-}\left(\left(E_{1}^{1 / 2} \alpha\right)\left(D_{1}^{+} u\right)\right)
$$

where $D_{1}^{+}$is the forward difference operator in the $x_{1}$ direction, $D_{1}^{-}$is the backward difference operator, and $E_{1}^{1 / 2}$ is the averaging operator

$$
\left(E_{1}^{1 / 2} \alpha\right)_{l, m}=\alpha_{l+1 / 2, m}=\frac{1}{2}\left(\alpha_{l+1, m}+\alpha_{l, m}\right) .
$$


Explicitly,

$$
\begin{aligned}
D_{1}^{-}\left(\left(E_{1}^{1 / 2} \alpha\right)\left(D_{1}^{+} u\right)\right) & =\frac{1}{h} D_{1}^{-}\left(\alpha_{l+1 / 2, m}\left(u_{l+1, m}-u_{l, m}\right)\right) \\
& =\frac{1}{h^{2}}\left(\alpha_{l+1 / 2, m}\left(u_{l+1, m}-u_{l, m}\right)-\alpha_{l-1 / 2, m}\left(u_{l, m}-u_{l-1, m}\right)\right) .
\end{aligned}
$$

Terms of the form $\partial_{2}\left(\alpha \partial_{2} u\right)$ are defined similarly:

$$
\partial_{2}\left(\alpha \partial_{2} u\right) \simeq D_{2}^{-}\left(\left(E_{2}^{1 / 2} \alpha\right)\left(D_{2}^{+} u\right)\right)
$$

For derivatives of the form $\partial_{2}\left(\beta \partial_{1} u\right)$, we use centered first-order differences $D_{1}^{0}$ and $D_{2}^{0}$,

$$
\begin{aligned}
\partial_{2}\left(\beta \partial_{1} u\right) & \simeq D_{2}^{0}\left(\beta\left(D_{1}^{0} u\right)\right) \\
& =\frac{1}{4 h^{2}}\left(\beta_{l, m+1}\left(u_{l+1, m+1}-u_{l-1, m+1}\right)-\beta_{l, m-1}\left(\left(u_{l+1, m-1}-u_{l-1, m-1}\right)\right)\right.
\end{aligned}
$$

and $\partial_{1}\left(\beta \partial_{2} u\right)$ is approximated in the same manner. When we wish to be brief, we will use the notation $P_{k, h}$ to denote the discretized spatial partial differential operator just defined.

For derivatives in time, we use the second-order accuate, centered difference, and we now have the scheme

$$
\begin{aligned}
u^{n+1}=2 u^{n}-u^{n-1}+k^{2} & {\left[D_{1}^{-}\left(\left(E_{1}^{1 / 2}(\lambda+2 \mu)\right)\left(D_{1}^{+} u^{n}\right)\right)\right.} \\
& \left.+D_{2}^{-}\left(\left(E_{2}^{1 / 2} \mu\right)\left(D_{2}^{+} u^{n}\right)\right)+D_{1}^{0}\left(\lambda\left(D_{2}^{0} v^{n}\right)\right)+D_{2}^{0}\left(\mu\left(D_{1}^{0} v^{n}\right)\right)\right] \\
v^{n+1}=2 v^{n}-v^{n-1}+k^{2} & {\left[D_{1}^{-}\left(\left(E_{1}^{1 / 2} \mu\right)\left(D_{1}^{+} v^{n}\right)\right)\right.} \\
& \left.+D_{2}^{-}\left(\left(E_{2}^{1 / 2}(\lambda+2 \mu)\right)\left(D_{2}^{+} v^{n}\right)\right)+D_{1}^{0}\left(\mu\left(D_{2}^{0} u^{n}\right)\right)+D_{2}^{0}\left(\lambda\left(D_{1}^{0} u^{n}\right)\right)\right] .
\end{aligned}
$$

Stability of this scheme is discussed in [25]; we omit further discussion here, but note that for the case of constant $\lambda, \mu$, the stability condition is

$$
k \leq \frac{h}{\sqrt{c_{P}^{2}+c_{S}^{2}}}=\frac{h}{\sqrt{\lambda+3 \mu}} .
$$

\subsubsection{Forward problem}

To compute $\Lambda_{f}$, we find the solution to the forward problem (1.1), using the scheme given above. Because computing $u^{n+1}$ only depends on $u^{n}$ and $u^{n-1}$, we only store values of $u$ for three time steps, updating as necessary. We will store these values in arrays $u[1], u[2], u[3]$, 
and we will store the computed boundary data in an array $\Lambda_{f}[n]$ where $n=1, \ldots, T / k-1$. For computing the first time step, we use the Taylor series expansion

$$
\begin{aligned}
u(k) & =u(0)+k u^{\prime}(0)+\frac{1}{2} k^{2} u^{\prime \prime}(0)+O\left(k^{3}\right) \\
& =u(0)+k u^{\prime}(0)+\frac{1}{2} k^{2} P u(0)+O\left(k^{3}\right) .
\end{aligned}
$$

Since we have assumed zero initial velocity, we then have the scheme

$$
\begin{aligned}
u^{1} & =u^{0}+\frac{1}{2} k^{2} P_{k, h} u^{0} \\
u^{n+1} & =2 u^{n}-u^{n-1}+k^{2} P_{k, h} u^{n} ; n \geq 2 .
\end{aligned}
$$

The program is summarized below in Algorithm (1).

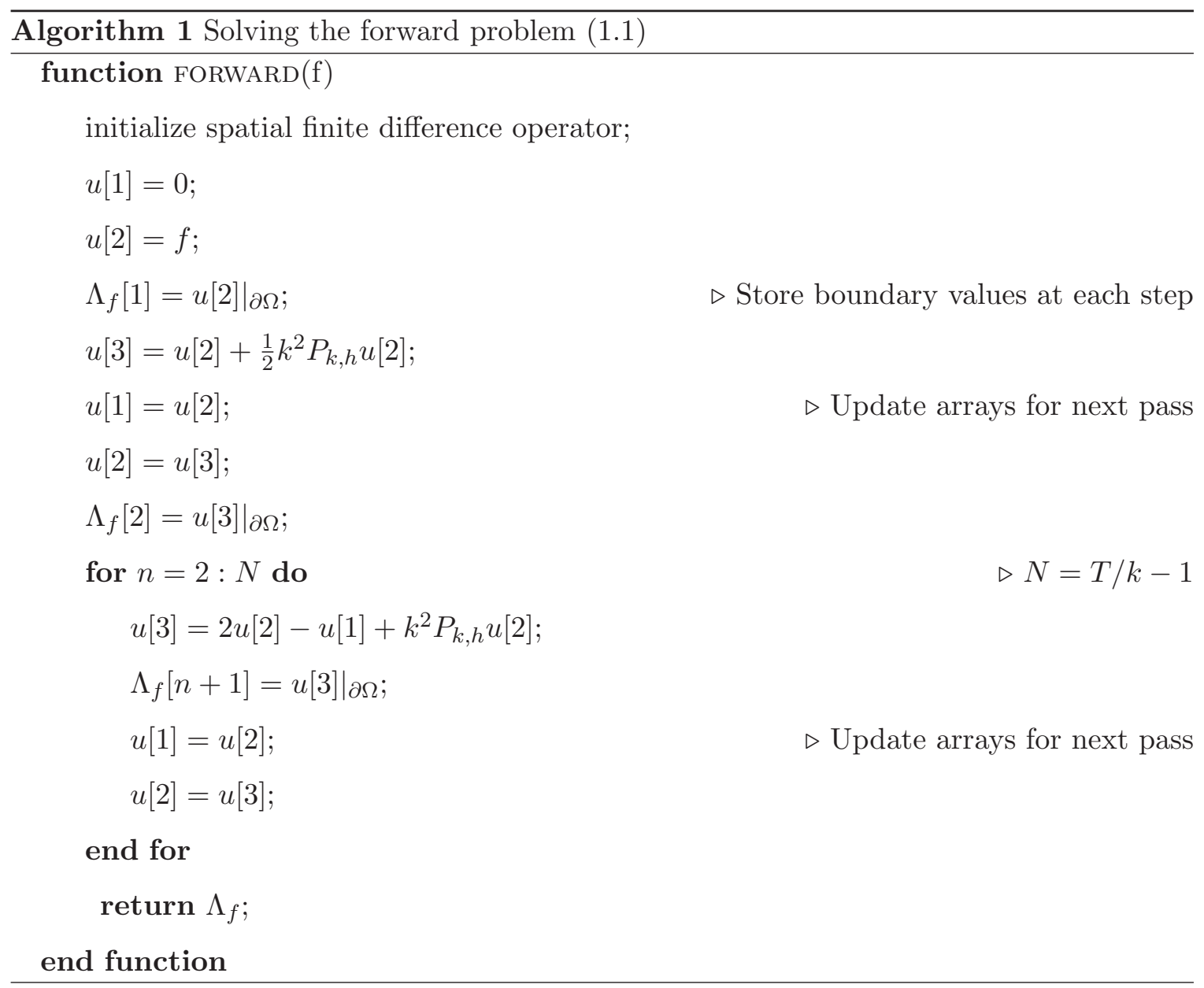




\subsubsection{Elliptic problem}

We will write $\phi=\phi_{1}, \psi=\phi_{2}$ to simplify notation here. To solve the elliptic PDE, we use Gauss-Seidel iteration. To do this, we start by writing $P_{k, h} \phi=0$ as a finite difference equation, and then solve for $\phi_{i}(l, m)$ when it appears. Explicitly, our method calls for us to expand terms like $\partial_{1}\left(\alpha \partial_{1} \phi\right)$ (where $\alpha=\lambda+2 \mu$, e.g.) as

$$
\begin{aligned}
\partial_{1}\left(\alpha \partial_{1} \phi\right) & \simeq \frac{1}{h^{2}}\left(\alpha_{l+1 / 2, m}\left(\phi_{l+1, m}-\phi_{l, m}\right)+\alpha_{l-1 / 2, m}\left(\phi_{l, m}-\phi_{l-1, m}\right)\right) \\
& =\frac{1}{h^{2}}\left(\alpha_{l-1 / 2, m}-\alpha_{l+1 / 2, m}\right) \phi_{l, m}+\frac{1}{h^{2}} \alpha_{l+1 / 2, m} \phi_{l+1, m}-\frac{1}{h^{2}} \alpha_{l-1 / 2, m} \phi_{l-1, m} .
\end{aligned}
$$

Terms of the form $\partial_{2}\left(\beta \partial_{1} \psi\right)$ are expanded as

$$
\partial_{2}\left(\beta \partial_{1} \psi\right) \simeq \frac{1}{4 h^{2}}\left(\beta_{l, m+1}\left(\psi_{l+1, m+1}-\psi_{l-1, m+1}\right)-\beta_{l, m-1}\left(\left(\psi_{l+1, m-1}-\psi_{l-1, m-1}\right)\right),\right.
$$

and then for the other terms in the equation

$$
\partial_{1}\left(\alpha_{1} \partial_{1} \phi\right)+\partial_{2}\left(\alpha_{2} \partial_{2} \phi\right)+\partial_{1}\left(\beta_{1} \partial_{2} \psi\right)+\partial_{2}\left(\beta_{2} \partial_{1} \psi\right)=0
$$

Then, we solve for $\phi_{l, m}$ in (4.1) to get an expression of the form

$$
\phi_{l, m}=F_{\phi}(\lambda, \mu, \phi, \psi, l, m)
$$

where

$$
\begin{gathered}
F_{\phi}:=\frac{1}{\gamma}\left[(\lambda+2 \mu)_{l+1 / 2, m} \phi_{l+1, m}-(\lambda+2 \mu)_{l-1 / 2, m} \phi_{l-1, m}+\mu_{l, m+1 / 2} \phi_{l, m+1}-\mu_{l, m-1 / 2} \phi_{l, m-1}\right. \\
+\frac{1}{4}\left(\lambda_{l+1, m}\left(\psi_{l+1, m+1}-\psi_{l+1, m-1}\right)-\lambda_{l-1, m}\left(\left(\psi_{l-1, m+1}-\psi_{l-1, m-1}\right)\right)\right. \\
+\frac{1}{4}\left(\mu_{l, m+1}\left(\psi_{l+1, m+1}-\psi_{l-1, m+1}\right)-\mu_{l, m-1}\left(\left(\psi_{l+1, m-1}-\psi_{l-1, m-1}\right)\right)\right] \\
\gamma=(\lambda+2 \mu)_{l+1 / 2, m}-(\lambda+2 \mu)_{l-1 / 2, m}+\mu_{l, m+1 / 2}-\mu_{l, m-1 / 2} .
\end{gathered}
$$

We proceed similarly with our second equation to get an expression for $\psi_{l, m}=F_{\psi}$, and then solve iteratively. Specifically, we use the method outlined in Algorithm 2, setting $\phi$ and $\psi$ equal to $\Lambda_{f}$ on $\partial \Omega$, and then iterating over our grid, updating the values of $\phi$ and $\lambda$ in terms of the expressions $F_{\phi}$ and $F_{\psi}$ derived above. Of course, since in each pass, the newly-computed value of a particular $\phi_{l, m}$ depends only on its directly adjacent neighbors, we will have to repeat the iteration over the grid many times, but eventually, this converges to the solution of (3.6). 


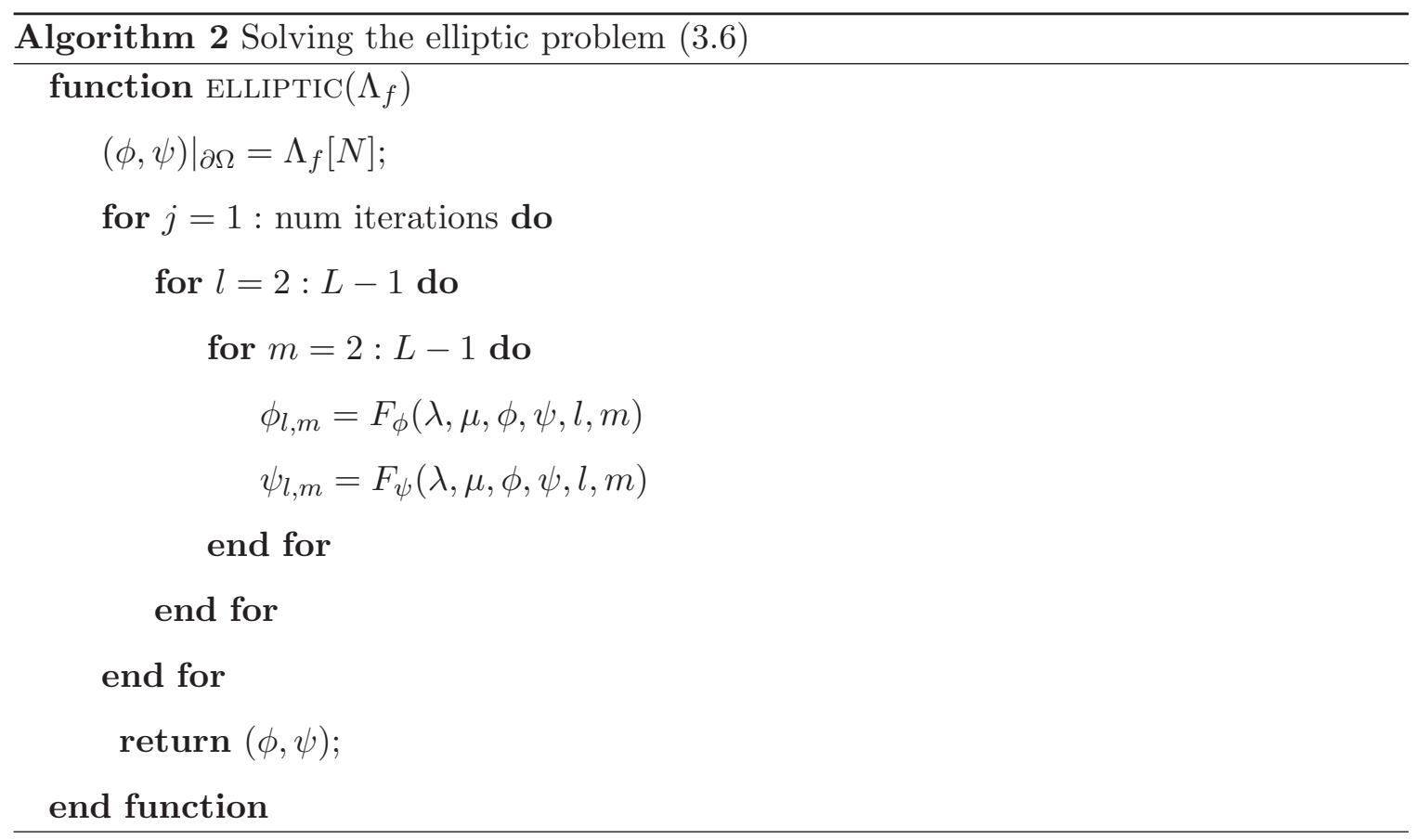

\subsubsection{Time-reversed problem}

We conclude this chapter by discussing our algorithm for the reverse-time problem (3.7). We use the same finite difference scheme as we did to solve (1.1), but we do this on a smaller grid, and instead of storing boundary values at each step, we use the previously computed boundary values to set $\left.v(\cdot, t)\right|_{\partial \Omega}$ at each step. Further, since our stencil is only 3 points wide (i.e., $v_{l, m}^{n}$ is computed in terms of values of $u$ and $v$ at directly adjacent grid points), we do not need to further modify our scheme to accomodate the boundary. This is outlined in Algorithm 3. 


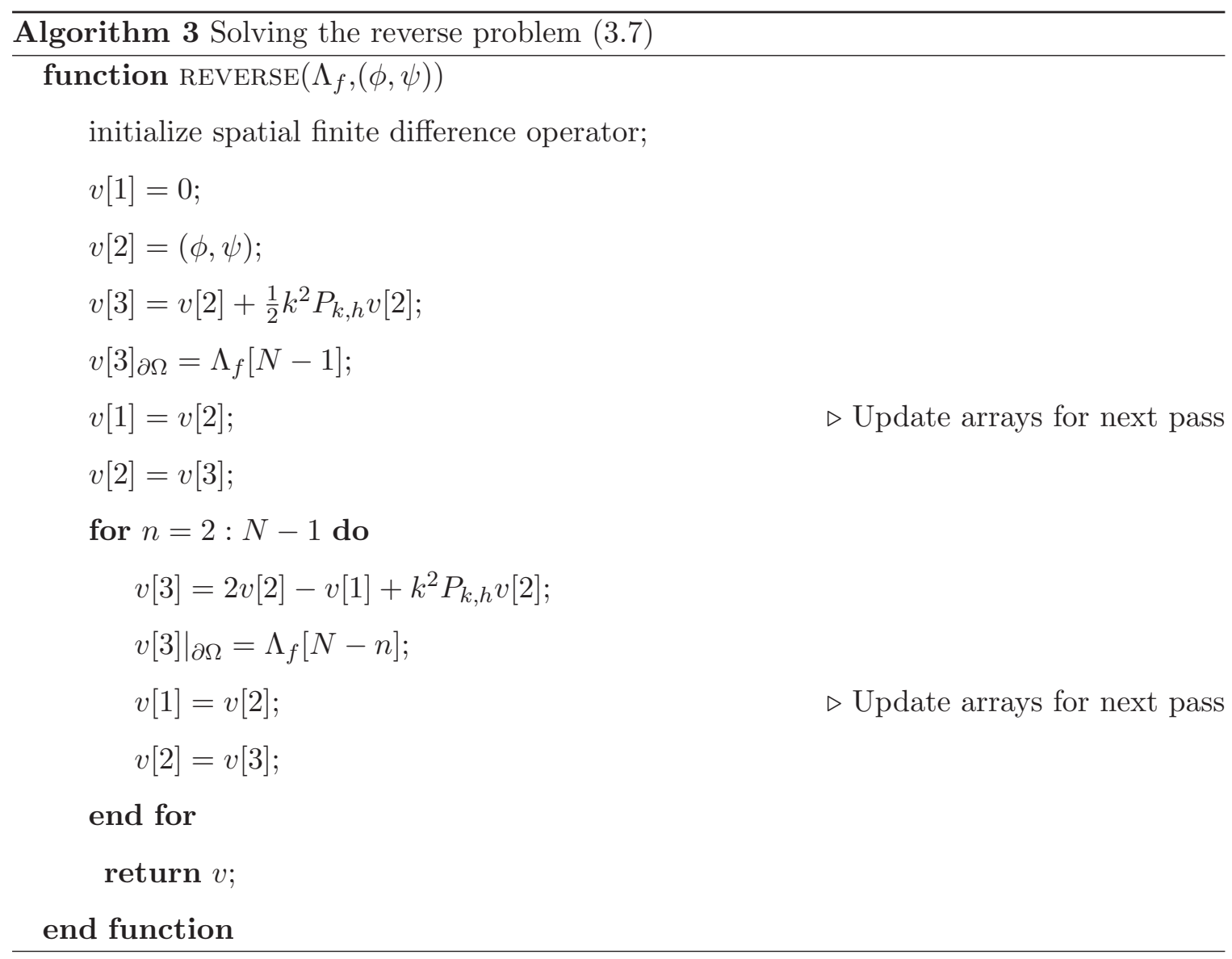


Chapter 5

\section{NUMERICAL WORK}

Having surveyed finite difference methods and developed schemes to solve (1.1), (3.7) and (3.6), we begin our numerical experimentation and implement the Neumann series method outlined in earlier chapters. We wish to explore the isotropic case, examining first the case where the two speeds satisfy the Lamé hypothesis outlined in earlier chapters, followed by the case where this condition is violated. We will see that, in both cases the algorithm performs reasonably well.

We additionally wish to explore the anisotropic case; this has not yet been explored theoretically, but, as it is no more computationally difficult than the isotropic case, we treat it here. We will see that the problem is tractable as well.

\subsection{Methodology}

Throughout the remainder of this work, we will pose our problem in two spatial dimensions, in which case, the operator $P$ is given by

$$
\begin{gathered}
(P u)_{1}=\partial_{1}\left((\lambda+2 \mu) \partial_{1} u_{1}\right)+\partial_{2}\left(\mu \partial_{2} u_{1}\right)+\partial_{1}\left(\lambda \partial_{2} u_{2}\right)+\partial_{2}\left(\mu \partial_{1} u_{2}\right) \\
(P u)_{2}=\partial_{2}\left(\lambda \partial_{1} u_{1}\right)+\partial_{1}\left(\mu \partial_{2} u_{1}\right)+\partial_{2}\left((\lambda+2 \mu) \partial_{2} u_{2}\right)+\partial_{1}\left(\mu \partial_{1} u_{2}\right)
\end{gathered}
$$

where $\lambda$ and $\mu$ will vary according to the needs of our numerical experimentation. In particular, we will start by considering the sets of parameters

$$
\begin{aligned}
& \lambda_{1}=0.25, \mu_{1}=1 ; \\
& \lambda_{2}=0.25\left(1+0.2 \sin \left(2 \pi x_{1}\right)+0.1 \cos \left(2 \pi x_{2}\right)\right)^{2}, \\
& \mu_{2}=0.25\left(2+0.1 \cos \left(2 \pi x_{1}\right)+0.2 \sin \left(2 \pi x_{2}\right)\right)^{2} ; \\
& \lambda_{3}=3.5, \mu_{3}=0.25\left(1+0.2 \sin \left(2 \pi x_{1}\right)+0.1 \cos \left(2 \pi x_{2}\right)\right)^{2} ; \\
& \lambda_{4}=0.25\left(2+0.2 \sin \left(2 \pi x_{1}\right)+0.1 \cos \left(2 \pi x_{2}\right)\right)^{2}, \mu_{4}=0.25(0.001)^{2} ;
\end{aligned}
$$




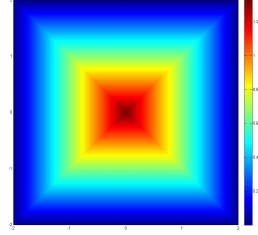

(a)

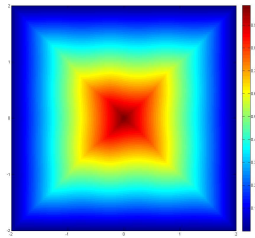

(c)

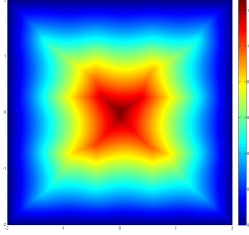

(b)

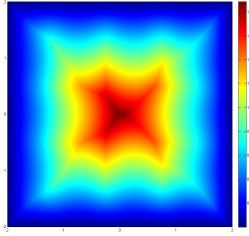

(d)

Figure 5.1: P-wave boundary distance maps for (a) $c_{1, P}: T_{0}=1.330$; (b) $c_{2, P}: T_{0}=1.261$; (c) $c_{3, P}: T_{0}=0.986 ;$ (d) $c_{4, P}: T_{0}=1.888$.

inside $\Omega$, and smoothly cutoff to be constant outside $\Omega$. We will refer to the corresponding $\mathrm{P}$-wave maximums and $\mathrm{S}$-wave minimums as $c_{i}^{+}, c_{i}^{-}$respectively. At this point, we note

$$
\begin{aligned}
& c_{1}^{+}=1.5, \quad c_{1}^{-}=1 \\
& c_{2}^{+}=1.751, \quad c_{2}^{-}=0.85 ; \\
& c_{3}^{+}=2.084, \quad c_{3}^{-}=0.35 ; \\
& c_{4}^{+}=1.150, \quad c_{4}^{-}=0.0005
\end{aligned}
$$

so that $\left(\lambda_{i}, \mu_{i}\right)$ satisfy the hypotheses of Theorem 3.2.4 for $i=1,2$, but for $i=3,4$, they do not. The homogeneous parameters $\left(\lambda_{1}, \mu_{1}\right)$ are included as a benchmark, and for $\left(\lambda_{4}, \mu_{4}\right)$, the $\mathrm{S}$-wave speed is on the order of $\frac{1}{1000}$ of the $\mathrm{P}$-wave speed. In this final case, we only run the simulation long enough for the P-wave to reach the boundary. 


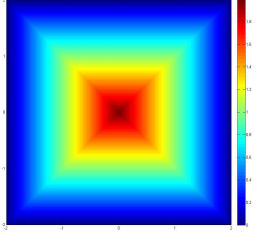

(a)

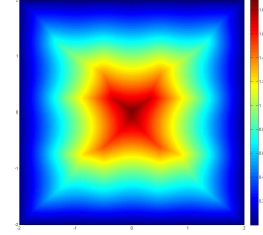

(b)

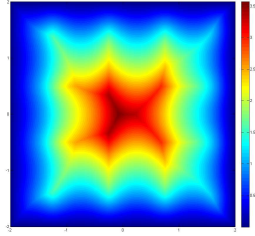

(c)

Figure 5.2: S-wave boundary distance maps for (a) $c_{1, S}: T_{1}=1.996$; (b) $c_{2, S}: T_{1}=1.888$; (c) $c_{3, S}: T_{1}=3.572$.

\subsubsection{Boundary Distance Map}

In order to estimate an appropriate simulation runtime $T$, we begin by computing the critical time $T_{0}$ by solving the eikonal equation

$$
\begin{aligned}
& |\nabla \widetilde{T}|=1 / c, \\
& \left.\widetilde{T}\right|_{\partial \Omega}=0,
\end{aligned}
$$

where $c=c_{i, P}=\sqrt{\lambda_{i}+2 \mu_{i}}$ is the $\mathrm{P}$-wave speed for the parameters in question. The solution $\widetilde{T}(x)$ represents the length of the shortest geodesic from $x$ to $\partial \Omega$. We then have

$T_{0}=\max _{\{x \in \Omega\}} \widetilde{T}$. We solve this equation using the fast-sweeping Lax-Friedrichs scheme outlined in [21] (see also [27], [46]).

In Figure 5.1, we show the boundary distance maps we have computed for these sets of parameters. The critical times are, respectively, 1.330,1.261, 0.986, 1.888, giving us minimum times the simulation must run in order to guarantee uniqueness.

We will also find it useful to compute the boundary distance functions for the S-wave speed $c=c_{i, S}=\sqrt{\mu_{i}}$. Intuitively, this is the minimum time needed to ensure that an 
S-wave signal from each point reaches the boundary. These boundary distance maps are shown in Figure 5.2, and the critical times (which we will refer to as $T_{1}$ ) are, respectively, $1.996,1.888,3.572$ for the first three sets of parameters. In the case of the fourth set, we do not need to determine $T_{1}$, as we will not attempt to run the simulation long enough for the S-wave to arrive (we would have $T_{1} \simeq 4000$ in this case!).

\subsubsection{Algorithmic Implementation}

The algorithm essentially consists of three steps: starting with initial data $f=\left(f_{1}, f_{2}\right)$, first simulate the forward solution of (1.1) and collect the boundary data $\Lambda_{f}$. Next, using $\Lambda_{f}(T, \cdot)$, solve the elliptic boundary value problem (3.6) to compute $\phi=\left(\phi_{1}, \phi_{2}\right)$. Finally, compute the solution of the reverse-time boundary value problem (3.7) with the elliptic solution $\phi$ as initial data (at $t=T$ ), and boundary data prescribed by $\Lambda_{f}$. When $t=0$, the solution of this reverse-time problem is the first term of the Neumann series. To compute successive terms, we can repeat the process. The program is outlined in algorithm 4, and specifics on the finite difference schemes used are found in the previous chapter.

Throughout, we take $\Omega=[-2,2]^{2}$, with initial data $f$ supported in $[-1.5,1.5]^{2}$. For solving the forward problem (1.1) (using forward), we use a 2nd order method, as outlined in the previous chapter (see also [22], [25]). In choosing to implement the forward problem this way, we introduce the problem of the computational boundary possibly causing reflection, and so we will take our computational domain to be $[-5,5]^{2}$ to begin, so that it is large enough to mitigate this issue. An alternate means of addressing this is the method of perfectly matched layers (used in [26], see also [2]), but we found that results and performance were not as good.

To address a potential inverse crime (where results could be overly optimistic if the simulation too closely matches the reconstruction) we use a finer grid for the initial simulation, and a coarser one for the remainder of the process. In particular, we will first take our grid spacing to be $h=0.005$, with the initial data sampled on a $600 \times 600$ grid, and time step $k=0.001$, and then $h=0.01, k=0.002$, so that the result is $300 \times 300$. Of course, these considerations necessarily increase the error in the process; error due to the finite 


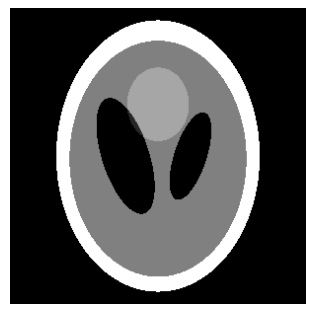

(a)

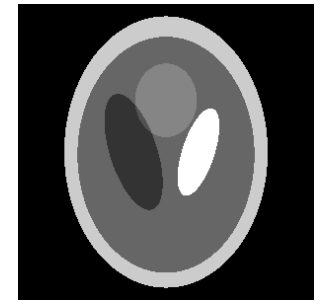

(b)

Figure 5.3: Initial Data

difference method (especially at the boundary) will not undo itself, and additional error will be introduced by downsampling $\Lambda_{f}$ when the grid is resized.

Here, we also note that, in each case $\sqrt{\left(c_{P}^{+}\right)^{2}+\left(c_{S}^{+}\right)^{2}} \leq 3$, so that we have

$$
k=\frac{1}{5} h \leq \frac{h}{\sqrt{\left(c_{P}^{+}\right)^{2}+\left(c_{S}^{+}\right)^{2}}}
$$

for each set of parameters, and so we can expect stability.

As discussed in the previous chapter, to solve (3.6), we use a Gauss-Seidel method (elliptic), and solving (3.7) is done using the same 2nd order method as forward, with the imposed Dirichlet boundary data (reverse).

\subsection{Results of Numerical Experimentation}

\subsubsection{Homogeneous parameters $\left(\lambda_{1}, \mu_{1}\right)$}

We first examine the case where the medium is homogeneous; i.e., the Lamé parameters are constant. Here, and throughout, we will use simplified versions of the Shepp-Logan phantom as our initial data, as seen in Figure 5.3. We will use one of the phantoms as $f_{1}$, the initial displacement in the $x_{1}$ direction, and the other as $f_{2}$, the $x_{2}$ displacement.

In Figure 5.4, we see the results of reconstruction with $T=4\left(\simeq 2 T_{1}\right)$. The error for our approximations to $f_{1}$ and $f_{2}$ are respectively $20.70 \%$ and $19.34 \%$ for the $m=0$ term, and $20.53 \%$ and $19.10 \%$ for the $m=1$ term (i.e. after 2 iterations), with error not decreasing substantially after this point (this data is compiled in Table 5.1). 


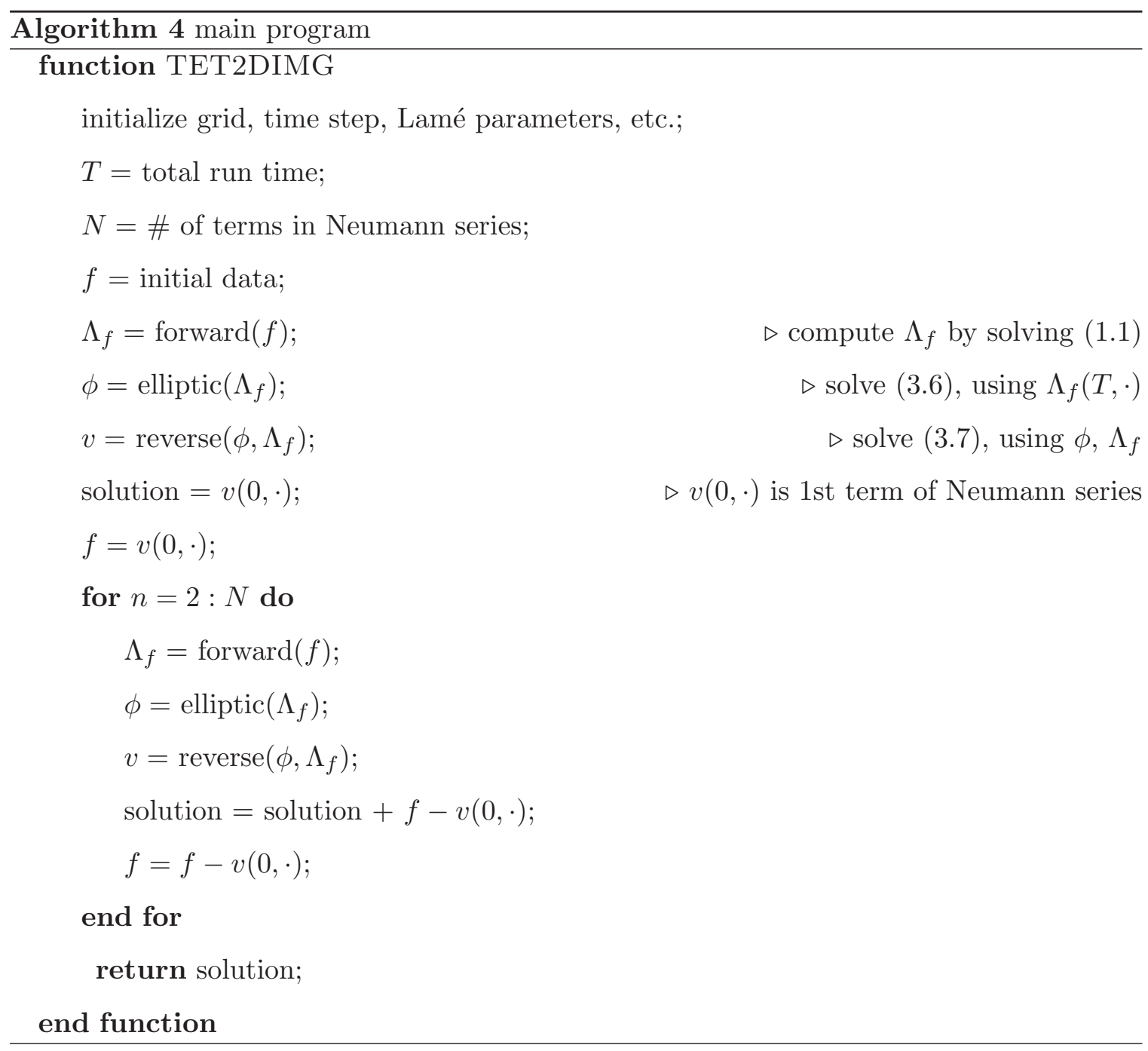




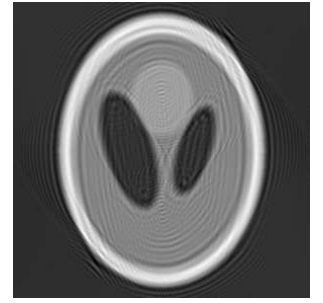

(a) $u_{1}, m=0$

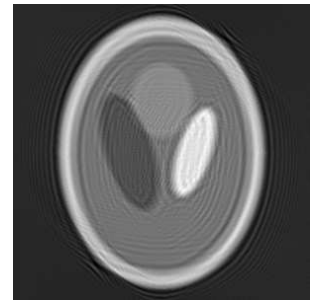

(d) $u_{2}, m=0$

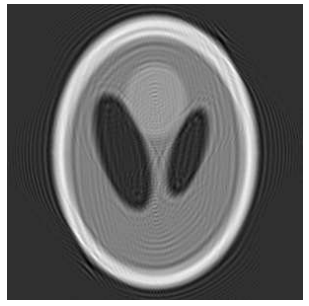

(b) $u_{1}, m=1$

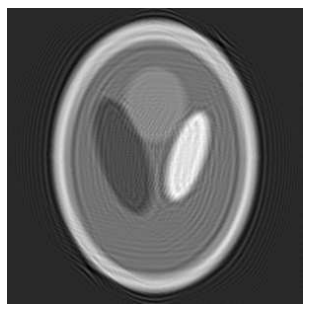

(e) $u_{2}, m=1$

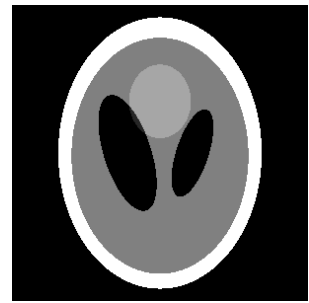

(c) Initial Data $f_{1}$

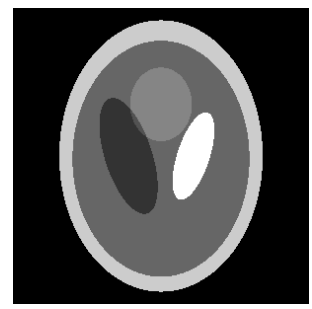

(f) Initial Data $f_{2}$

Figure 5.4: $\left(\lambda_{1}, \mu_{1}\right)$

Next, we add $10 \%$ noise to our data; the results can be seen in Figure 5.5. Error is described in Table 5.1; we notice that error is actually not increased by noising $\Lambda_{f}$; this is possibly because the total error due to finite difference approximations and using different meshes is of the same order as the introduced noise.

\subsubsection{Heterogeneous parameters $\left(\lambda_{2}, \mu_{2}\right)$}

We now examine the results when $\lambda$ and $\mu$ are allowed to vary. Again, we take $T=4 \simeq 2 T_{1}$. Reconstruction is still reasonable, though the error is higher, as expected. Results are shown below in Figure 5.6, with corresponding errors in Table 5.2. In Figure 5.7, we see the effect of adding $10 \%$ noise. It is interesting to note that error only decreases after the second iteration, and can actually become slightly worse, or at least does not show substantial improvement, in successive approximations. Again, this is possibly an effect of the finite difference approximation and differing meshes limiting the efficacy of the Neumann series. We next try adding $20 \%$ noise (not shown), and can see in Table 5.2 that, once again, overall 


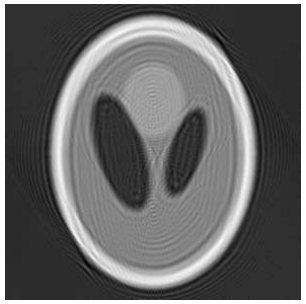

(a) $u_{1}, m=0$

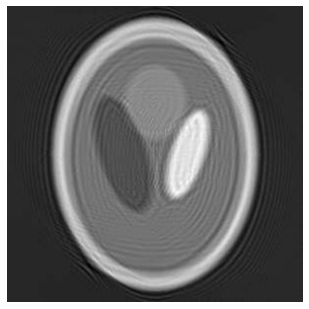

(d) $u_{2}, m=0$

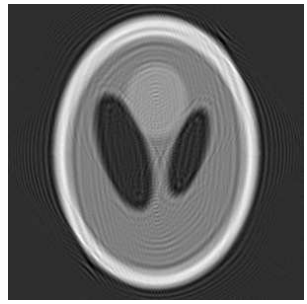

(b) $u_{1}, m=1$

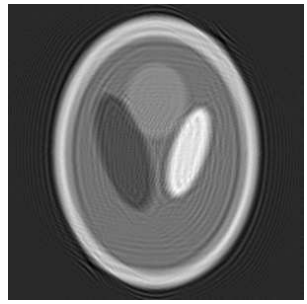

(e) $u_{2}, m=1$

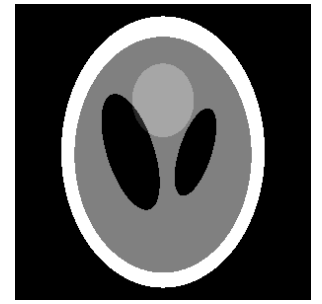

(c) Initial Data $f_{1}$

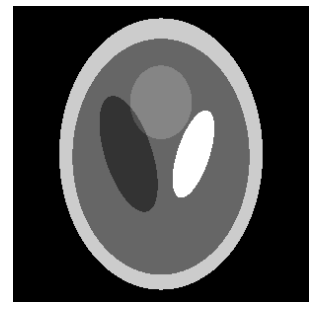

(f) Initial Data $f_{2}$

Figure 5.5: $\left(\lambda_{1}, \mu_{1}\right), 10 \%$ noise

\begin{tabular}{|c|c|c|c|}
\cline { 3 - 4 } \multicolumn{2}{c|}{} & \multicolumn{2}{c|}{$\mathrm{m}$} \\
\cline { 3 - 4 } \multicolumn{2}{c|}{} & 0 & 1 \\
\hline \multirow{2}{*}{ No noise } & $u_{1}$ & $20.70 \%$ & $20.53 \%$ \\
\cline { 2 - 4 } & $u_{2}$ & $19.34 \%$ & $19.10 \%$ \\
\hline \multirow{2}{*}{$10 \%$ noise } & $u_{1}$ & $20.78 \%$ & $20.63 \%$ \\
\cline { 2 - 4 } & $u_{2}$ & $19.43 \%$ & $19.20 \%$ \\
\hline
\end{tabular}

Table 5.1: error for $\left(\lambda_{1}, \mu_{1}\right)$ 


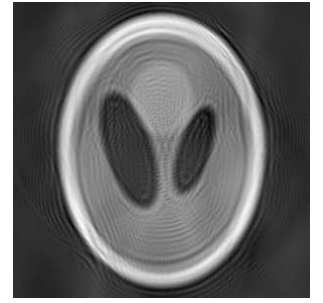

(a) $u_{1}, m=0$

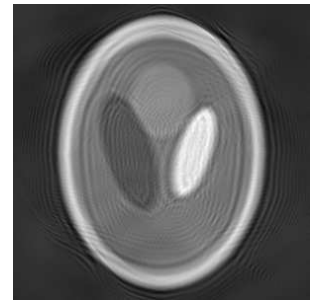

(d) $u_{2}, m=0$

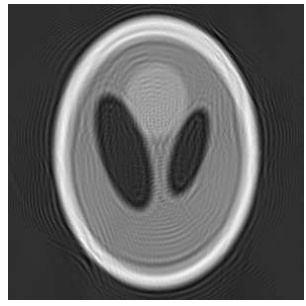

(b) $u_{1}, m=1$

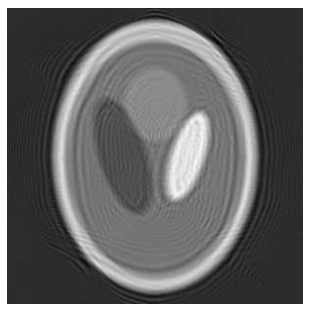

(e) $u_{2}, m=1$

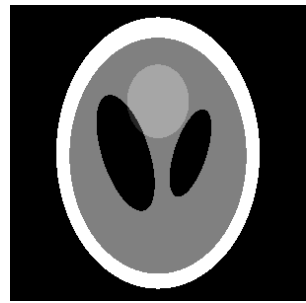

(c) Initial Data $f_{1}$

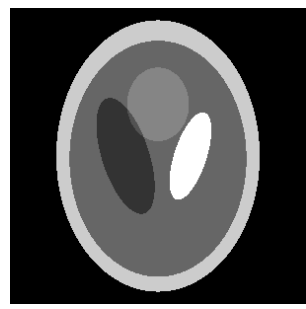

(f) Initial Data $f_{2}$

Figure 5.6: $\left(\lambda_{2}, \mu_{2}\right)$

error is not substantially increased.

\subsubsection{Heterogeneous parameters $\left(\lambda_{3}, \mu_{3}\right)$, Lamé hypothesis violated}

Next, we wish to explore the case where the conditions of Theorem 3.2.4 are not satisfied, in part to gain intuition whether or not these are necessary conditions. Because the value of $T_{1}$ for this set of parameters is roughly double the values for the previous sets, we will double the runtime of our simulation to $T=8$ (the results of running the simulation with only $T=4$ can be seen in Figure 5.8). In this case, it is also necessary to increase the size of the computational domain (to $[-12,12]^{2}$ ) to prevent problems with our computational boundary. That is, we want to ensure that a signal originating in $\Omega$, and traveling at $c_{3}^{+}$ will not be able to reflect off the artificial boundary and reenter $\Omega$.

In Figure 5.9, we see the results of the reconstruction, with error in Table 5.3. Results are not as good as in the previous two cases, but identification of the phantom's main features is still possible. Once again, error does not improve past the second term, probably 


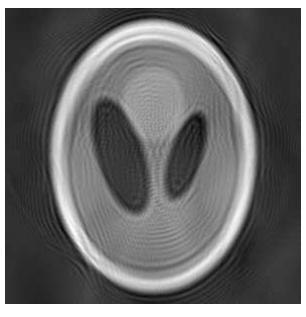

(a) $u_{1}, m=0$

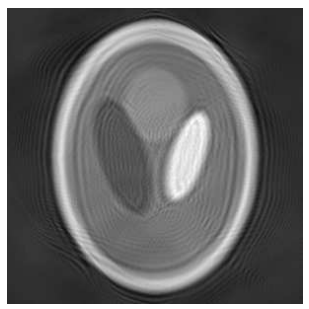

(d) $u_{2}, m=0$

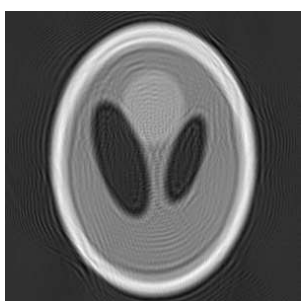

(b) $u_{1}, m=1$

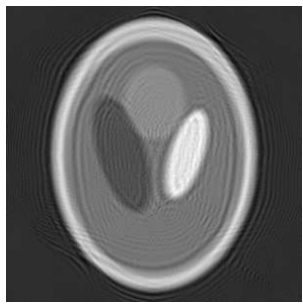

(e) $u_{2}, m=1$

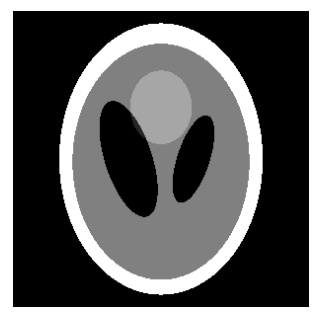

(c) Initial Data $f_{1}$

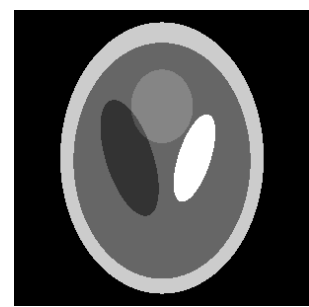

(f) Initial Data $f_{2}$

Figure 5.7: $\left(\lambda_{2}, \mu_{2}\right), 10 \%$ noise

\begin{tabular}{|c|c|c|c|c|c|c|}
\cline { 3 - 7 } \multicolumn{2}{c|}{} & \multicolumn{5}{c|}{$\mathrm{m}$} \\
\cline { 3 - 7 } \multicolumn{2}{c|}{} & 0 & 1 & 2 & 3 & 4 \\
\hline \multirow{2}{*}{ No noise } & $u_{1}$ & $23.62 \%$ & $20.83 \%$ & $20.85 \%$ & $20.92 \%$ & $20.96 \%$ \\
\cline { 2 - 7 } & $u_{2}$ & $20.87 \%$ & $19.46 \%$ & $19.50 \%$ & $19.55 \%$ & $19.58 \%$ \\
\hline \multirow{2}{*}{$10 \%$ noise } & $u_{1}$ & $23.69 \%$ & $20.93 \%$ & $20.96 \%$ & $21.04 \%$ & $21.08 \%$ \\
\cline { 2 - 7 } & $u_{2}$ & $20.95 \%$ & $19.56 \%$ & $19.61 \%$ & $19.67 \%$ & $19.71 \%$ \\
\hline \multirow{2}{*}{$20 \%$ noise } & $u_{1}$ & $23.92 \%$ & $21.27 \%$ & $21.34 \%$ & $21.44 \%$ & $21.49 \%$ \\
\cline { 2 - 7 } & $u_{2}$ & $21.19 \%$ & $19.87 \%$ & $19.97 \%$ & $20.05 \%$ & $20.09 \%$ \\
\hline
\end{tabular}

Table 5.2: error for $\left(\lambda_{2}, \mu_{2}\right)$ 


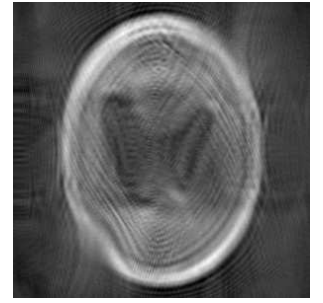

(a) $u_{1}, m=0$

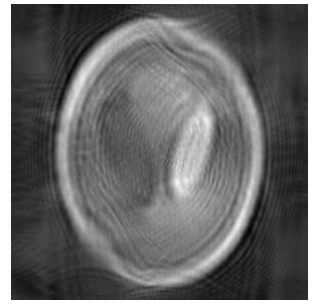

(d) $u_{2}, m=0$

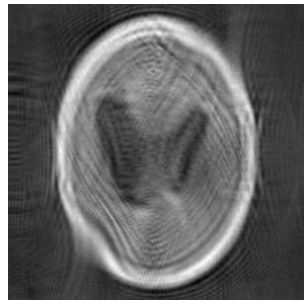

(b) $u_{1}, m=1$

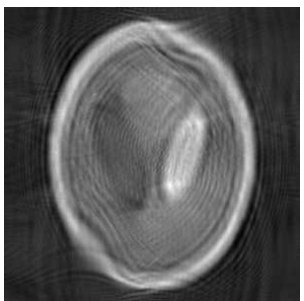

(e) $u_{2}, m=1$

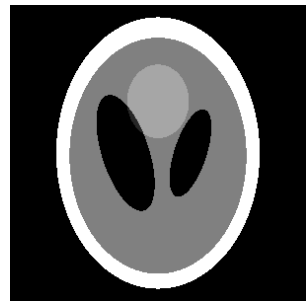

(c) Initial Data $f_{1}$

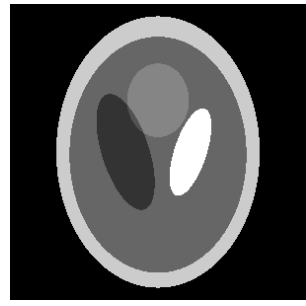

(f) Initial Data $f_{2}$

Figure 5.8: $\left(\lambda_{3}, \mu_{3}\right), T=4$

\begin{tabular}{|c|c|c|c|c|c|c|}
\cline { 3 - 7 } \multicolumn{2}{c|}{} & \multicolumn{5}{c|}{$\mathrm{m}$} \\
\cline { 2 - 7 } \multicolumn{2}{c|}{} & 0 & 1 & 2 & 3 & 4 \\
\hline \multirow{3}{*}{ No noise } & $u_{1}$ & $30.45 \%$ & $27.99 \%$ & $27.91 \%$ & $27.91 \%$ & $27.91 \%$ \\
\cline { 2 - 7 } & $u_{2}$ & $26.26 \%$ & $24.28 \%$ & $24.16 \%$ & $24.15 \%$ & $24.15 \%$ \\
\hline
\end{tabular}

Table 5.3: error for $\left(\lambda_{3}, \mu_{3}\right), T=8$

limited by our finite difference scheme and different meshes. If we are willing to potentially commit inverse crime, we can run the forward simulation on the same mesh as the successive reconstructions, and view the results (Figure 5.10 and Table 5.4 ) without the downsampling error and with less finite difference error. Here, the reconstruction is much better, and error continues to decrease with additional passes. If we add noise, the error increases, but in keeping with the type of stability we expect. 


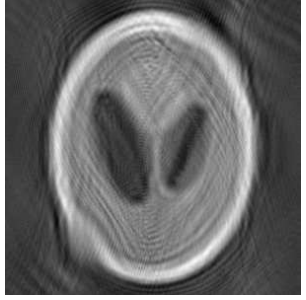

(a) $u_{1}, m=0$

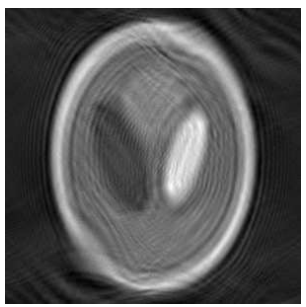

(d) $u_{2}, m=0$

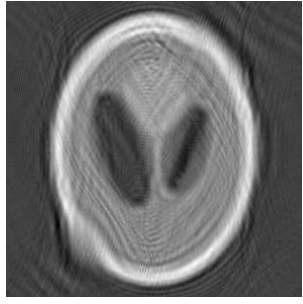

(b) $u_{1}, m=1$

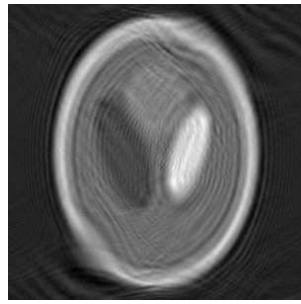

(e) $u_{2}, m=1$

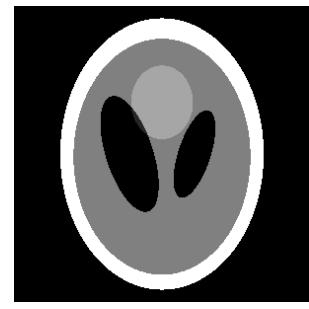

(c) Initial Data $f_{1}$

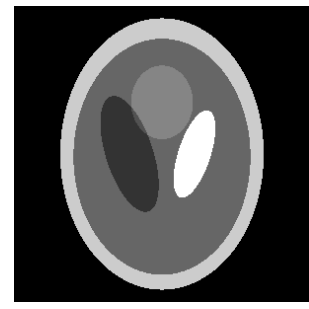

(f) Initial Data $f_{2}$

Figure 5.9: $\left(\lambda_{3}, \mu_{3}\right), T=8$

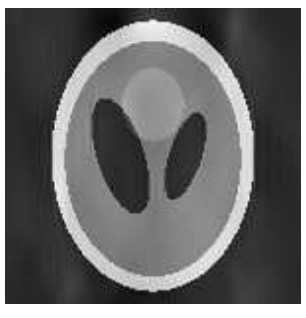

(a) $u_{1}, m=0$

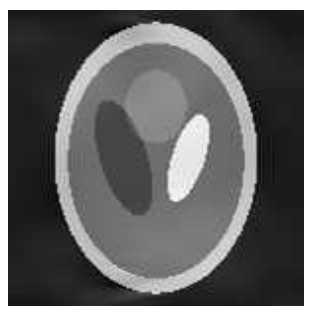

(d) $u_{2}, m=0$

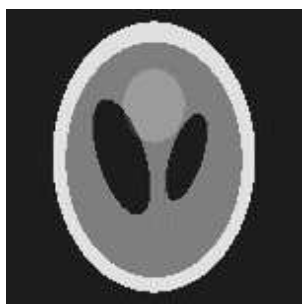

(b) $u_{1}, m=4$

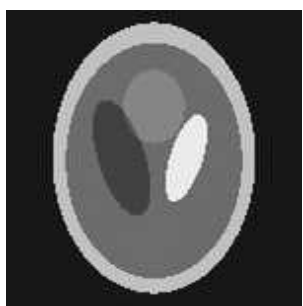

(e) $u_{2}, m=4$

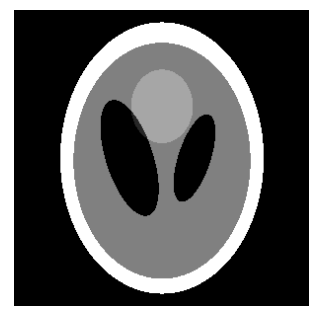

(c) Initial Data $f_{1}$

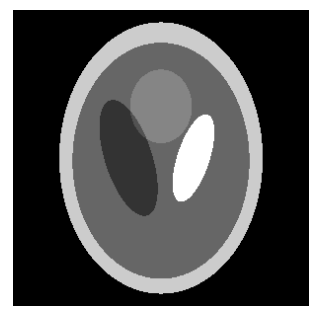

(f) Initial Data $f_{2}$

Figure 5.10: $\left(\lambda_{3}, \mu_{3}\right), T=8$, same mesh 

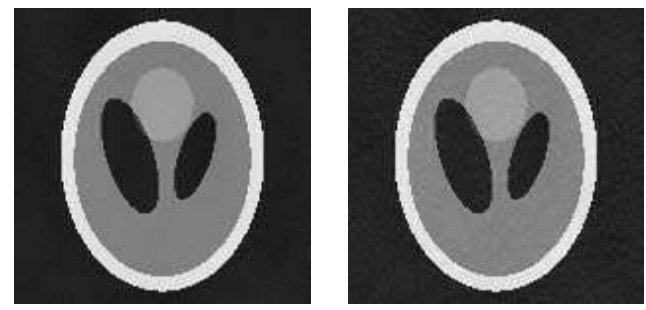

(a) $u_{1}, m=1,10 \%$

(b) $u_{1}, m=1,20 \%$

noise

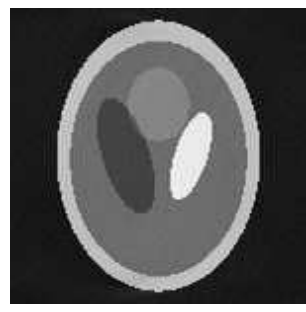

noise

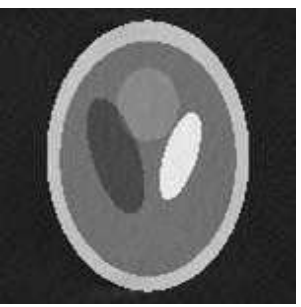

(d) $u_{2}, m=1,10 \%$

noise (e) $u_{2}, m=1,20 \%$

noise

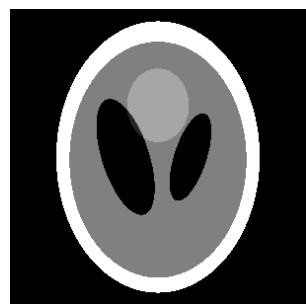

(c) Initial Data $f_{1}$

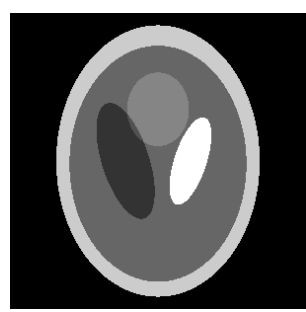

(f) Initial Data $f_{2}$

Figure 5.11: $\left(\lambda_{3}, \mu_{3}\right), T=8$, added noise, same mesh

\begin{tabular}{|c|c|c|c|c|c|c|}
\cline { 3 - 7 } \multicolumn{2}{c|}{} & \multicolumn{5}{|c|}{$\mathrm{m}$} \\
\cline { 2 - 7 } \multicolumn{2}{c|}{} & 0 & 1 & 2 & 3 & 4 \\
\hline \multirow{2}{*}{ No noise } & $u_{1}$ & $12.52 \%$ & $5.88 \%$ & $5.25 \%$ & $5.08 \%$ & $4.99 \%$ \\
\cline { 2 - 7 } & $u_{2}$ & $10.61 \%$ & $6.02 \%$ & $5.40 \%$ & $5.25 \%$ & $5.20 \%$ \\
\hline \multirow{2}{*}{$10 \%$ noise } & $u_{1}$ & $12.99 \%$ & $6.86 \%$ & $6.35 \%$ & $6.21 \%$ & $6.15 \%$ \\
\cline { 2 - 7 } & $u_{2}$ & $11.12 \%$ & $6.88 \%$ & $6.33 \%$ & $6.21 \%$ & $6.17 \%$ \\
\hline \multirow{2}{*}{$20 \%$ noise } & $u_{1}$ & $14.37 \%$ & $9.18 \%$ & $8.83 \%$ & $8.75 \%$ & $8.71 \%$ \\
\cline { 2 - 7 } & $u_{2}$ & $12.49 \%$ & $8.92 \%$ & $8.53 \%$ & $8.45 \%$ & $8.43 \%$ \\
\hline
\end{tabular}

Table 5.4: error for $\left(\lambda_{3}, \mu_{3}\right), T=8$, same mesh 


\subsubsection{Heterogeneous parameters $\left(\lambda_{4}, \mu_{4}\right)$, Lamé hypothesis violated with $c_{S} \ll c_{P}$}

We conclude this section by exploring the situation where the $\mathrm{P}$-wave speed is much greater than that of the $\mathrm{S}$-wave, and we choose $\lambda$ and $\mu$ so that $c_{P}$ is roughly 1000 times $c_{S}$. In this situation, we will only run the simulation long enough to measure the P-wave at the boundary; indeed, even if we wanted to measure the S-wave, we would have to run the simulation so long that the P-wave would reflect off any reasonable artificial boundary (i.e., the grid would have to be so large, the program's runtime would be impractically long, and memory requirements would exceed the computer's resources). Because of this, we cannot expect to recover the full initial data, but hopefully we can still recover the singularities of the data; that is, we attempt to image an "outline" of the phantom's main features.

The results of this experiment can be seen in 5.12), and we omit an error table (as we really only expect our results to give information on a set of measure 0 ). We see that the boundaries of the main regions within the phantom can be identified, though the amplitudes are not well-recovered. Results improve slightly after one iteration $(m=1)$, but actually start to become much worse with successive passes. With noise, the results are about the same (not shown).

\subsection{Anisotropic Elasticity}

Finally, we briefly test an anisotropic elastic system. For the general case in 2 dimensions, there are 6 independent coefficients $c_{i j}$ in the system of PDEs, with $1 \leq i, j \leq 3$ and $c_{i j}=c_{j i}$. Here, we treat the special orthotropic case, where $c_{13}=c_{23}=0\left(=c_{31}=c_{32}\right)$, and the system can be written

$$
\begin{aligned}
& (P u)_{1}=\partial_{1}\left(c_{11} \partial_{1} u_{1}\right)+\partial_{2}\left(c_{33} \partial_{2} u_{1}\right)+\partial_{1}\left(c_{12} \partial_{2} u_{2}\right)+\partial_{2}\left(c_{33} \partial_{1} u_{2}\right) \\
& (P u)_{2}=\partial_{2}\left(c_{12} \partial_{1} u_{1}\right)+\partial_{1}\left(c_{33} \partial_{2} u_{1}\right)+\partial_{2}\left(c_{22} \partial_{2} u_{2}\right)+\partial_{1}\left(c_{33} \partial_{1} u_{2}\right)
\end{aligned}
$$

(note that isotropic systems are the special case where $c_{11}=c_{22}=c_{12}+2 c_{33}$ ). Physically, this is a material with two orthogonal axes of symmetry, sometimes called transversally 


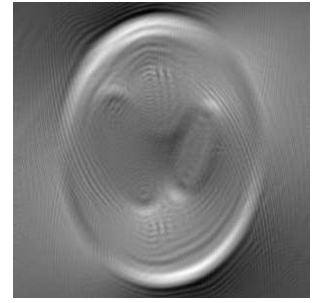

(a) $u_{1}, m=0$

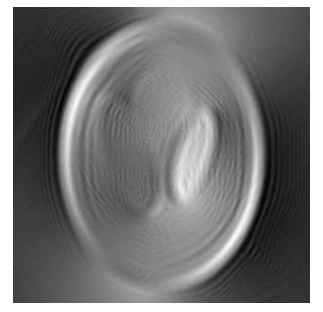

(d) $u_{2}, m=0$

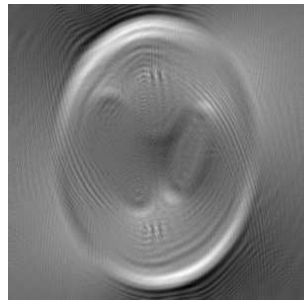

(b) $u_{1}, m=1$

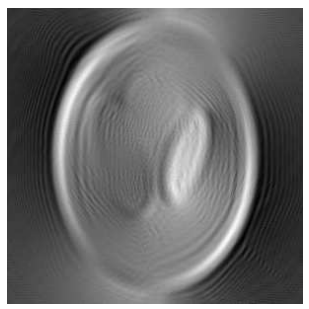

(e) $u_{2}, m=1$

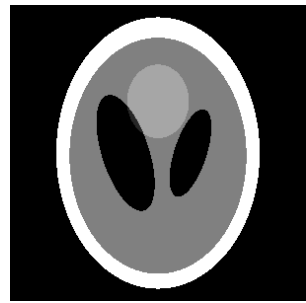

(c) Initial Data $f_{1}$

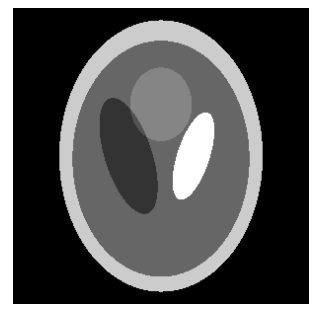

(f) Initial Data $f_{2}$

Figure 5.12: $\left(\lambda_{4}, \mu_{4}\right), T=4$

isotropic. We choose our parameters so that

$$
\begin{aligned}
& c_{12}=0.25\left(1+0.2 \sin \left(2 \pi x_{1}\right)+0.1 \cos \left(2 \pi x_{2}\right)\right)^{2}, \\
& c_{33}=0.25\left(2+0.1 \cos \left(2 \pi x_{1}\right)+0.2 \sin \left(2 \pi x_{2}\right)\right)^{2}, \\
& c_{11}=c_{12}+2 c_{33}+0.25, \\
& c_{22}=c_{12}+2 c_{33}-0.25 .
\end{aligned}
$$

It is not yet known whether one can show that a Neumann-series approach works, but from the standpoint of our program, it is no harder to implement.

Results are seen Figures 5.13 and 5.14, with error for both recorded in Table 5.5. The outcome and error of these experiments is comparable to that of $\left(\lambda_{2}, \mu_{2}\right)$, which is not entirely surprising, as this is an anisotropic perturbation of those parameters. The experiment responds similarly to noise in this case as well. Of course, this is only a "mild" anisotropy, and there remain plenty of testing to be done, but this seems to be a preliminary indication that some kind of reconstruction process can work for anisotropic systems. 


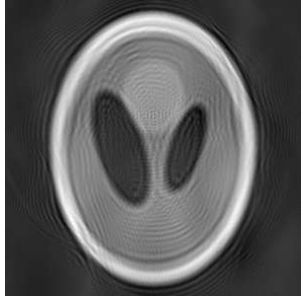

(a) $u_{1}, m=0$

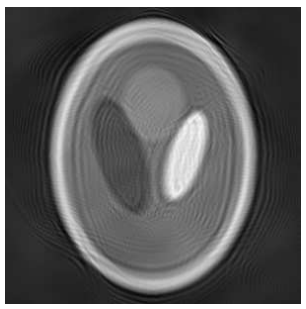

(d) $u_{2}, m=0$

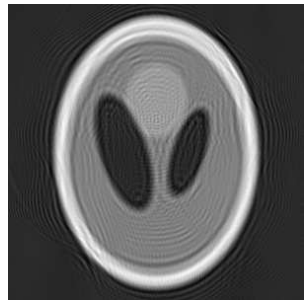

(b) $u_{1}, m=1$

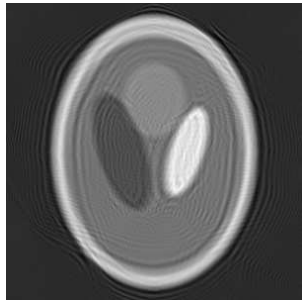

(e) $u_{2}, m=1$

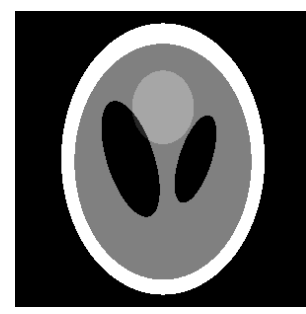

(c) Initial Data $f_{1}$

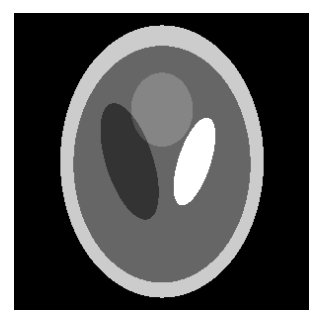

(f) Initial Data $f_{2}$

Figure 5.13: Anisotropic system

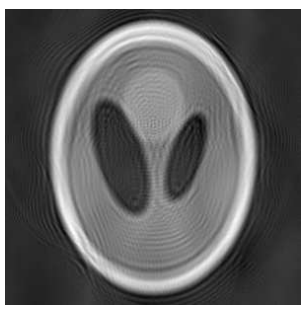

(a) $u_{1}, m=0$

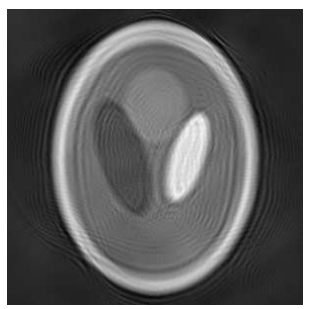

(d) $u_{2}, m=0$

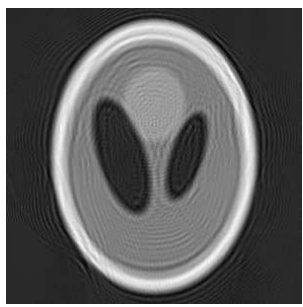

(b) $u_{1}, m=1$

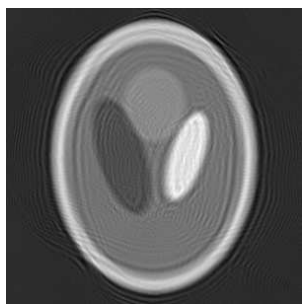

(e) $u_{2}, m=1$

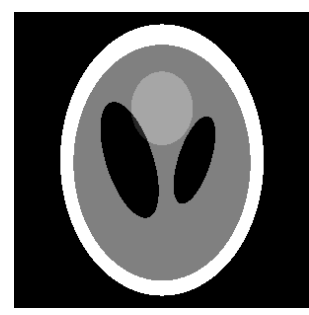

(c) Initial Data $f_{1}$

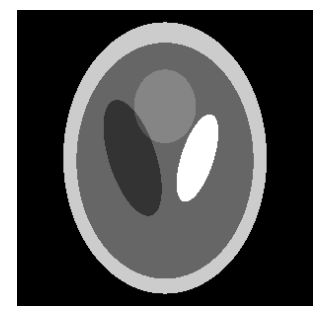

(f) Initial Data $f_{2}$

Figure 5.14: Anisotropic system with $10 \%$ noise 


\begin{tabular}{|c|c|c|c|c|c|c|}
\cline { 3 - 7 } \multicolumn{2}{c|}{} & \multicolumn{5}{c|}{$\mathrm{m}$} \\
\cline { 2 - 7 } \multicolumn{2}{c|}{} & 0 & 1 & 2 & 3 & 4 \\
\hline \multirow{3}{*}{ No noise } & $u_{1}$ & $23.28 \%$ & $20.83 \%$ & $20.86 \%$ & $20.92 \%$ & $20.96 \%$ \\
\cline { 2 - 7 } & $u_{2}$ & $20.96 \%$ & $19.43 \%$ & $19.49 \%$ & $19.55 \%$ & $19.59 \%$ \\
\hline \multirow{3}{*}{$10 \%$ noise } & $u_{1}$ & $23.37 \%$ & $20.94 \%$ & $20.99 \%$ & $21.06 \%$ & $21.10 \%$ \\
\cline { 2 - 7 } & $u_{2}$ & $21.06 \%$ & $19.56 \%$ & $19.63 \%$ & $19.69 \%$ & $19.73 \%$ \\
\hline
\end{tabular}

Table 5.5: error for anisotropic system

\subsection{Conclusion}

While we have suggested and implemented a method of reconstruction for thermoacoustic tomography in elastic media, and demonstrated the uniqueness and stability of this method, there is still much work to be done.

Indeed, we have only explored the case of complete data, and in practical applications, such as breast imaging and geophysical imaging, one can only expect to be able to measure data on a portion of the boundary. Thus, examining the problem of incomplete data will be critical to the practical relevance of the elastic approach, and should be explored in future work. As previously mentioned, various authors (again, see [31], [44], [45]) have investigated this problem for the acoustic wave equation, and produced satisfactory results; perhaps by adapting these methods, good results can be obtained for setting of elastic media as well.

Furthermore, we have worked with the restriction that $c^{+} / 3<c^{-}$, which, as mentioned, is a reasonable hypothesis for earth materials, but not necessarily for biological tissue. This assumption may be stricter than actually needed, and so it would be nice to find an alternative to using [7] in the uniqueness step. At this point, it is not clear how to accomplish this, but perhaps by adapting the methods (rather than the results) of Eller, Isakov, Nakamura and Tataru some progress can be made. Indeed, in their work the coefficients $a_{j}$ are generally allowed to vary in time and be of relatively limited smoothness (e.g., $C^{1}$ ); possibly by relaxing these assumptions, a better unique continuation result can be obtained.

Regarding our numerical experimentation, in comparison to the homogeneous bench- 
mark, our program performs about as well on heterogeneous parameters that satisfy the Lamé parameter hypothesis given in Chapter 3. While results are somewhat worse if the condition is violated, the main features of the phantom are still easily recognizable. Even in the case where the $\mathrm{S}$-wave is several orders of magnitude slower than the $\mathrm{P}$-wave (and so is not measured), the outlines of these features remain visible.

At this time, results seem limited by accuracy of finite difference method (especially at the boundary), and error in downsampling $\Lambda_{f}$ when the grid is resized. If we want to avoid a potential inverse crime, the second type of error is unavoidable, though the first issue could be mitigated by either increasing the accuracy of the finite difference method (to, say, fourth-order), or increasing the resolution of the grid. While a finer grid is easy to implement, running time and memory requirements would suffer greatly, as these grow like $n^{3}$ with respect to the resolution of the grid. Thus, a more accurate scheme may prove the most useful in future work. One consequence of this seemingly inherent error is that the program's results generally did not improve after the second pass (i.e., after computing the $m=0$ and $m=1$ terms).

In all cases, the program responded to noise with the kind of stability expected; that is, perturbing the measured data by $10 \%$ did not have significant consequences with regard to the reconstruction. In fact, in most cases, the noise did not seem to make a significant contribution to the overall error, and was most likely hidden by the error inherent to our process. In the cases we ran without inverse crime considerations, we saw a greater contribution to the error from noising, though it still behaved in a stable way.

Finally, the program seems to do a reasonable job of reconstruction for the anisotropic case, though only a single case has been treated here. It is likely that there are types of anisotropy that do not respond as well to this program's approach, and it would be useful to know what criteria distinguish these cases. 


\section{BIBLIOGRAPHY}

[1] M. Agranovsky, C. Berenstein and P. Kuchment. Approximation by spherical waves in $L^{p}$-spaces. J. Geom. Anal. 6, (3):365-383. 1996.

[2] D. Appelö and G. Kreiss. A new absorbing layer for elastic waves. Journal of Computational Physics. 215, (2):642-660. 2006.

[3] V. Brytik, M. V. de Hoop, H. F. Smith and G. Uhlmann. Decoupling of modes for the elastic wave equation in media of limited smoothness. Commun. Partial Differ. Eqns. 36 1683-93. 2011.

[4] P. Burgholzer, G. J. Matt, M. Haltmeier and G. Paltauf. Exact and approximate imaging methods for photoacoustic tomography using an arbitrary detection surface. Physical Reviews E $\mathbf{7 5}$ 046706. 2007.

[5] J. Cheng, V. Isakov, M. Yamamoto and Q. Zhou. Lipschitz stability in the lateral Cauchy problem for elasticity system. J. Math. Kyoto Univ. 43-3, 475 - 501. 2003.

[6] J.J. Duistermaat. Fourier integral operators, (Progress in Mathematics). Birkhäuser. 1995.

[7] M. Eller, V. Isakov, G. Nakamura and D. Tataru. Uniqueness and stability in the Cauchy problem for Maxwell's and elasticity systems. College de France Seminar, 14, 'Studies in Math. Appl.', Vol.31, North-Holland, Elsevier Science 329 - 349. 2002.

[8] D. Finch, M. Haltmeier and Rakesh. Inversion of spherical means and the wave equation in even dimensions. SIAM J. Appl. Math. 68, Issue 2, 392-412. 2007.

[9] D. Finch, S. Patch and Rakesh. Determining a function from its mean values over a family of spheres. SIAM J. Math. Anal. 351213 - 1240. 2004.

[10] G. Friedlander and M. Joshi. Introduction to the Theory of Distributions. Cambridge University Press. 1982.

[11] A. Grigis and J. Sjöstrand. Microlocal analysis for differential operators: An introduction, (London Mathematical Society Lecture Note Series). Cambridge University Press. 1994.

[12] L. Hörmander. The Analysis of Linear Partial Differential Operators I. Springer-Verlag, Berlin. 1985. 
[13] L. Hörmander.The Analysis of Linear Partial Differential Operators II. SpringerVerlag, Berlin. 1985.

[14] L. Hörmander.The Analysis of Linear Partial Differential Operators III. SpringerVerlag, Berlin. 1985.

[15] Y. Hristova. Time reversal in thermoacoustic tomography - an error estimate. Inverse Problems 25 055008. 2009.

[16] Y. Hristova, P. Kuchment and L. Nguyen. On reconstruction and time reversal in thermoacoustic tomography in homogeneous and non-homogeneous acoustic media. Inverse Problems 24 055006. 2008.

[17] P. Kuchment and L. Kunyansky. Mathematics of thermoacoustic tomography. European J. Appl. Math. 19, Issue 02, 191-224. 2008.

[18] P. Kuchment and L. Kunyansky. Mathematics of thermoacoustic and photoacoustic tomography. Chapter 19 in Vol. 2 of Handbook of Mathematical Methods in Imaging. Springer Verlag. pp.817 - 866. 2010.

[19] L. Kunyansky. A series solution and a fast algorithm for the inversion of the spherical mean Radon transform. Inverse Problems 23. 2007.

[20] L. Kunyansky. Explicit inversion formulas for the the spherical mean Radon transform. Inverse Problems 23. 2007.

[21] C. Y. Kao, S. J. Osher and J. Qian. Lax-Friedrichs sweeping schemes for static Hamilton-Jacobi equations. J. Comp. Phys., 196:367-391. 2004.

[22] K. R. Kelly, R. W. Ward, S. Treitel and R. M. Alford. Synthetic seismograms: A finite-difference approach. Geophysics. 41, (1):2-27. 1976.

[23] C-L. Lin, G. Nakamura, G. Uhlmann and J-N. Wang. Quantitative strong unique continuation for the Lamé system with less regular coefficients. Methods and Applications of Analysis 18, 85-92. 2011.

[24] J. McLaughlin and J. Yoon. Unique identifiability of elastic parameters from timedependent interior displacement measurement. Inverse Problems 2025 - 45. 2004.

[25] S. Nilsson, N. A. Petersson, B. Sjögreen and H-O Kreiss. Stable difference approximations for the elastic wave equation in second order formulation. SIAM J. Numer. Anal., 45 19021936. 2007. 
[26] J. Qian, P. Stefanov, G. Uhlmann and H-K. Zhao. An efficient Neumann-series based algorithm for thermoacoustic and photoacoustic tomography with a variable sound speed. SIAM Journal on Imaging Sciences 4, 850-883. 2011.

[27] J. Qian, Y. T. Zhang and H. K. Zhao. Fast sweeping methods for eikonal equations on triangulated meshes. SIAM J. Numer. Analy., 45:83-107. 2007.

[28] Reed and Simon. Functional Analysis, volume 1 of Methods of Modern Mathematical Physics. Academic Press. 1972.

[29] W. Rudin. Functional Analysis. McGraw-Hill. 1973.

[30] X. Saint-Raymond. Elementary Introduction to the Theory of Pseudodifferential Operators (Studies in Advanced Mathematics). CRC Press. 1991.

[31] P. Stefanov and G. Uhlmann. Thermoacoustic tomography with variable sound speed. Inverse Problems 25 075011. 2009.

[32] P. Stefanov and G. Uhlmann. Thermoacoustic tomography arising in brain imaging. Inverse Problems 27 045004. 2011.

[33] C.C. Stolk. On the modeling and inversion of seismic data. Ph.D. thesis, Utrecht University. 2000.

[34] J. Strikwerda. Finite Difference Schemes and Partial Differential Equations. SIAM. 2007.

[35] D. Tataru. Unique continuation for solutions to PDEs; between Hörmanders theorem and Holmgrens theorem. Comm. Partial Differential Equations 20 (5-6):855-884. 1995.

[36] D. Tataru. Unique continuation for operators with partially analytic coefficients. $J$. Math. Pures Appl. (9), 78(5):505-521. 1999.

[37] M. E. Taylor. Partial Differential Equations I. Basic Theory. Springer-Verlag. 1996.

[38] M. E. Taylor. Pseudodifferential Operators, volume 34 of Princeton Mathematical Series. Princeton University Press, Princeton, N.J. 1981.

[39] M. E. Taylor. Reflection of singularities of solutions to systems of differential equations. Comm. Pure Appl. Math. 28:457-478. 1975.

[40] J. Tittelfitz. Thermoacoustic tomography in elastic media. Inverse Problems 28055004. 2012. 
[41] F. Treves. Introduction to Pseudodifferential and Fourier Integral Operators, Volumes 1 and 2 (University Series in Mathematics). Springer. 1980.

[42] F. Treves. Topological Vector Spaces, Distributions and Kernels. Academic Press, New York. 1967.

[43] M. Xu and L. Wang. Universal back-projection algorithm for photoacoustic computed tomography. Physical Reviews E 71 016706. 2005.

[44] Y. Xu, P. Kuchment, and G. Ambartsoumian. Reconstructions in limited view thermoacoustic tomography. Medical Physics, 31(4):724-733. 2004.

[45] Y. Xu, L. Wang, P. Kuchment, and G. Ambartsoumian. Limited view thermoacoustic tomography. Photoacoustic imaging and spectroscopy, L.H. Wang (Editor), chapter 6, pages 61-73. CRC Press. 2009.

[46] H. K. Zhao. Fast sweeping method for eikonal equations. Math. Comp., 74:603-627. 2005. 